\title{
Shuffle-compatible permutation statistics II: the exterior peak set
}

\author{
Darij Grinberg \\ College of Science \& Engineering \\ University of Minnesota \\ Minneapolis, MN, U.S.A. \\ darijgrinberg@gmail.com
}

Submitted: Jun 20, 2018; Accepted: Oct 7, 2018; Published: Oct 19, 2018

(C) The author. Released under the CC BY license (International 4.0).

\begin{abstract}
This is a continuation of the work "Shuffle-compatible permutation statistics" by Gessel and Zhuang (but can be read independently from the latter). We study the shuffle-compatibility of permutation statistics - a concept introduced by Gessel and Zhuang, although various instances of it have appeared throughout the literature before. We prove that (as Gessel and Zhuang have conjectured) the exterior peak set statistic (Epk) is shuffle-compatible. We furthermore introduce the concept of an "LR-shuffle-compatible" statistic, which is stronger than shuffle-compatibility. We prove that Epk and a few other statistics are LR-shuffle-compatible. Furthermore, we connect these concepts with the quasisymmetric functions, in particular the dendriform structure on them.
\end{abstract}

Mathematics Subject Classifications: 05E05, 05A05, 06A11

This paper is a continuation of the work [4] by Gessel and Zhuang (but can be read independently from the latter). It is devoted to the study of shuffle-compatibility of permutation statistics - a concept introduced in [4], although various instances of it have appeared throughout the literature before.

In Section 1, we introduce the notations that we will need throughout this paper. In Section 2, we prove that the exterior peak set statistic Epk is shuffle-compatible (Theorem 60), as conjectured by Gessel and Zhuang in [4]. In Section 3, we introduce the concept of an "LR-shuffle-compatible" statistic, which is stronger than shuffle-compatibility. We give a sufficient criterion for it and use it to show that Epk and some other statistics are LR-shuffle-compatible.

The last three sections relate all of this to quasisymmetric functions; these sections are only brief summaries, and we refer to [7] for the details. In Section 4, we recall the concept of descent statistics introduced in [4] and its connection to quasisymmetric 
functions. Motivated by this connection, in Section 5, we define the kernel of a descent statistic, and study this kernel for Epk, giving two explicit generating sets for this kernel. In Section 6, we extend the quasisymmetric functions connection to the concept of LRshuffle-compatible statistics, and relate it to dendriform algebras.

\section{Remark on alternative versions}

This paper also has a detailed version [7], which includes some proofs that have been omitted from the present version as well as more details on some other proofs and further results in Sections 4, 5 and 6.

\section{Notations and definitions}

Let us first introduce the definitions and notations that we will use in the rest of this paper. Many of these definitions appear in [4] already; we have tried to deviate from the notations of [4] as little as possible.

\subsection{Permutations and other basic concepts}

Definition 1. We let $\mathbb{N}=\{0,1,2,3, \ldots\}$ and $\mathbb{P}=\{1,2,3, \ldots\}$. Both of these sets are understood to be equipped with their standard total order. Elements of $\mathbb{P}$ will be called letters (despite being numbers).

Definition 2. Let $n \in \mathbb{Z}$. We shall use the notation $[n]$ for the totally ordered set $\{1,2, \ldots, n\}$ (with the usual order relation inherited from $\mathbb{Z}$ ). Note that $[n]=\varnothing$ when $n \leqslant 0$.

Definition 3. Let $n \in \mathbb{N}$. An $n$-permutation shall mean a word with $n$ letters, which are distinct and belong to $\mathbb{P}$. Equivalently, an $n$-permutation shall be regarded as an injective map $[n] \rightarrow \mathbb{P}$ (the image of $i$ under this map being the $i$-th letter of the word).

For example, $(3,6,4)$ and $(9,1,2)$ are 3 -permutations, but $(2,1,2)$ is not.

Definition 4. A permutation is defined to be an $n$-permutation for some $n \in \mathbb{N}$. If $\pi$ is an $n$-permutation for some $n \in \mathbb{N}$, then the number $n$ is called the size of the permutation $\pi$ and is denoted by $|\pi|$. A permutation is said to be nonempty if it is nonempty as a word (i.e., if its size is $>0$ ).

Note that the meaning of "permutation" we have just defined is unusual (most authors define a permutation to be a bijection from a set to itself); we are following [4] in defining permutations this way.

Definition 5. Let $n \in \mathbb{N}$. Two $n$-permutations $\alpha$ and $\beta$ are said to be order-isomorphic if they have the following property: For every two integers $i, j \in[n]$, we have $\alpha(i)<\alpha(j)$ if and only if $\beta(i)<\beta(j)$. 


\section{Definition 6.}

(a) A permutation statistic is a map st from the set of all permutations to an arbitrary set that has the following property: Whenever $\alpha$ and $\beta$ are two order-isomorphic permutations, we have st $\alpha=$ st $\beta$.

(b) Let st be a permutation statistic. Two permutations $\alpha$ and $\beta$ are said to be stequivalent if they satisfy $|\alpha|=|\beta|$ and st $\alpha=$ st $\beta$. The relation "st-equivalent" is an equivalence relation; its equivalence classes are called st-equivalence classes.

Remark 7 . Let $n \in \mathbb{N}$. Let us call an $n$-permutation $\pi$ standard if its letters are $1,2, \ldots, n$ (in some order). The standard $n$-permutations are in bijection with the $n$ ! permutations of the set $\{1,2, \ldots, n\}$ in the usual sense of this word (i.e., the bijections from this set to itself).

It is easy to see that for each $n$-permutation $\sigma$, there exists a unique standard $n$ permutation $\pi$ order-isomorphic to $\sigma$. Thus, a permutation statistic is uniquely determined by its values on standard permutations. Consequently, we can view permutation statistics as statistics defined on standard permutations, i.e., on permutations in the usual sense of the word.

The word "permutation statistic" is often abbreviated as "statistic".

\subsection{Some examples of permutation statistics}

Definition 8. Let $n \in \mathbb{N}$. Let $\pi=\left(\pi_{1}, \pi_{2}, \ldots, \pi_{n}\right)$ be an $n$-permutation.

(a) The descents of $\pi$ are the elements $i \in[n-1]$ satisfying $\pi_{i}>\pi_{i+1}$.

(b) The descent set of $\pi$ is defined to be the set of all descents of $\pi$. This set is denoted by Des $\pi$, and is always a subset of $[n-1]$.

(c) The peaks of $\pi$ are the elements $i \in\{2,3, \ldots, n-1\}$ satisfying $\pi_{i-1}<\pi_{i}>\pi_{i+1}$.

(d) The peak set of $\pi$ is defined to be the set of all peaks of $\pi$. This set is denoted by $\operatorname{Pk} \pi$, and is always a subset of $\{2,3, \ldots, n-1\}$.

(e) The left peaks of $\pi$ are the elements $i \in[n-1]$ satisfying $\pi_{i-1}<\pi_{i}>\pi_{i+1}$, where we set $\pi_{0}=0$.

(f) The left peak set of $\pi$ is defined to be the set of all left peaks of $\pi$. This set is denoted by $\operatorname{Lpk} \pi$, and is always a subset of $[n-1]$. It is easy to see that (for $n \geqslant 2$ ) we have

$$
\operatorname{Lpk} \pi=\operatorname{Pk} \pi \cup\left\{1 \mid \pi_{1}>\pi_{2}\right\} .
$$

(The strange notation " $\left\{1 \mid \pi_{1}>\pi_{2}\right\}$ " means the set of all numbers 1 satisfying $\pi_{1}>\pi_{2}$. In other words, it is the 1-element set $\{1\}$ if $\pi_{1}>\pi_{2}$, and the empty set $\varnothing$ otherwise.) 
(g) The right peaks of $\pi$ are the elements $i \in\{2,3, \ldots, n\}$ satisfying $\pi_{i-1}<\pi_{i}>\pi_{i+1}$, where we set $\pi_{n+1}=0$.

(h) The right peak set of $\pi$ is defined to be the set of all right peaks of $\pi$. This set is denoted by $\operatorname{Rpk} \pi$, and is always a subset of $\{2,3, \ldots, n\}$. It is easy to see that (for $n \geqslant 2$ ) we have

$$
\operatorname{Rpk} \pi=\operatorname{Pk} \pi \cup\left\{n \mid \pi_{n-1}<\pi_{n}\right\} .
$$

(i) The exterior peaks of $\pi$ are the elements $i \in[n]$ satisfying $\pi_{i-1}<\pi_{i}>\pi_{i+1}$, where we set $\pi_{0}=0$ and $\pi_{n+1}=0$.

(j) The exterior peak set of $\pi$ is defined to be the set of all exterior peaks of $\pi$. This set is denoted by $\operatorname{Epk} \pi$, and is always a subset of $[n]$. It is easy to see that (for $n \geqslant 2$ ) we have

$$
\begin{aligned}
\operatorname{Epk} \pi & =\operatorname{Pk} \pi \cup\left\{1 \mid \pi_{1}>\pi_{2}\right\} \cup\left\{n \mid \pi_{n-1}<\pi_{n}\right\} \\
& =\operatorname{Lpk} \pi \cup \operatorname{Rpk} \pi
\end{aligned}
$$

(where, again, $\left\{n \mid \pi_{n-1}<\pi_{n}\right\}$ is the 1-element set $\{n\}$ if $\pi_{n-1}<\pi_{n}$, and otherwise is the empty set).

(For $n=1$, we have $\operatorname{Epk} \pi=\{1\}$.)

For example, the 6-permutation $\pi=(4,1,3,9,6,8)$ has

$$
\begin{aligned}
\operatorname{Des} \pi & =\{1,4\}, & & \operatorname{Pk} \pi=\{4\}, \\
\operatorname{Lpk} \pi=\{1,4\}, & & \operatorname{Rpk} \pi=\{4,6\}, & \operatorname{Epk} \pi=\{1,4,6\} .
\end{aligned}
$$

For another example, the 6-permutation $\pi=(1,4,3,2,9,8)$ has

$$
\begin{aligned}
\operatorname{Des} \pi & =\{2,3,5\}, & \operatorname{Pk} \pi & =\{2,5\}, \\
\operatorname{Lpk} \pi & =\{2,5\}, & \operatorname{Rpk} \pi=\{2,5\}, & \operatorname{Epk} \pi=\{2,5\} .
\end{aligned}
$$

Notice that Definition 8 actually defines several permutation statistics. For example, Definition 8 (b) defines the permutation statistic Des, whose codomain is the set of all subsets of $\mathbb{P}$. Likewise, Definition 8 (d) defines the permutation statistic Pk, and Definition 8 (f) defines the permutation statistic Lpk, whereas Definition 8 (h) defines the permutation statistic Rpk. The main permutation statistic that we will study in this paper is Epk, which is defined in Definition $8(\mathbf{j})$; its codomain is the set of all subsets of $\mathbb{P}$.

The following simple fact expresses the set $\operatorname{Epk} \pi$ corresponding to an $n$-permutation $\pi$ in terms of Des $\pi$ :

Proposition 9. Let $n$ be a positive integer. Let $\pi$ be an $n$-permutation. Then,

$$
\operatorname{Epk} \pi=(\operatorname{Des} \pi \cup\{n\}) \backslash(\operatorname{Des} \pi+1),
$$

where Des $\pi+1$ denotes the set $\{i+1 \mid i \in \operatorname{Des} \pi\}$.

Proof of Proposition 9. The rather easy proof can be found in the detailed version [7] of this paper. 


\subsection{Shuffles and shuffle-compatibility}

Definition 10. Let $\pi$ and $\sigma$ be two permutations.

(a) We say that $\pi$ and $\sigma$ are disjoint if no letter appears in both $\pi$ and $\sigma$.

(b) Assume that $\pi$ and $\sigma$ are disjoint. Set $m=|\pi|$ and $n=|\sigma|$. Let $\tau$ be an $(m+n)$ permutation. Then, we say that $\tau$ is a shuffle of $\pi$ and $\sigma$ if both $\pi$ and $\sigma$ are subsequences of $\tau$.

(c) We let $S(\pi, \sigma)$ be the set of all shuffles of $\pi$ and $\sigma$.

For example, the permutations $(3,1)$ and $(6,2,9)$ are disjoint, whereas the permutations $(3,1,2)$ and $(6,2,9)$ are not. The shuffles of the two disjoint permutations $(3,1)$ and $(2,6)$ are

$$
\begin{array}{lll}
(3,1,2,6), & (3,2,1,6), & (3,2,6,1), \\
(2,3,1,6), & (2,3,6,1), & (2,6,3,1) .
\end{array}
$$

If $\pi$ and $\sigma$ are two disjoint permutations, and if $\tau$ is a shuffle of $\pi$ and $\sigma$, then each letter of $\tau$ must be either a letter of $\pi$ or a letter of $\sigma$. (This follows easily from the pigeonhole principle.)

If $\pi$ and $\sigma$ are two disjoint permutations, then $S(\pi, \sigma)=S(\sigma, \pi)$ is an $\left(\begin{array}{c}m+n \\ m\end{array}\right)$ element set, where $m=|\pi|$ and $n=|\sigma|$.

Definition 10 (b) is used, e.g., in [5]. From the point of view of combinatorics on words, it is somewhat naive, as it fails to properly generalize to the case when the words $\pi$ and $\sigma$ are no longer disjoint ${ }^{1}$. But we will not be considering this general case, since our results do not seem to straightforwardly extend to it (although we might have to look more closely); thus, Definition 10 will suffice for us.

\section{Definition 11.}

(a) If $a_{1}, a_{2}, \ldots, a_{k}$ are finitely many arbitrary objects, then $\left\{a_{1}, a_{2}, \ldots, a_{k}\right\}_{\text {multi }}$ denotes the multiset whose elements are $a_{1}, a_{2}, \ldots, a_{k}$ (each appearing with the multiplicity with which it appears in the list $\left.\left(a_{1}, a_{2}, \ldots, a_{k}\right)\right)$.

(b) Let $\left(a_{i}\right)_{i \in I}$ be a finite family of arbitrary objects. Then, $\left\{a_{i} \mid i \in I\right\}_{\text {multi }}$ denotes the multiset whose elements are the elements of this family (each appearing with the multiplicity with which it appears in the family).

\footnotetext{
${ }^{1}$ In this general case, it is best to define a shuffle of two words $\pi=\left(\pi_{1}, \pi_{2}, \ldots, \pi_{m}\right)$ and $\sigma=\left(\sigma_{1}, \sigma_{2}, \ldots, \sigma_{n}\right)$ as a word of the form $\left(\gamma_{\eta(1)}, \gamma_{\eta(2)}, \ldots, \gamma_{\eta(m+n)}\right)$, where $\left(\gamma_{1}, \gamma_{2}, \ldots, \gamma_{m+n}\right)$ is the word $\left(\pi_{1}, \pi_{2}, \ldots, \pi_{m}, \sigma_{1}, \sigma_{2}, \ldots, \sigma_{n}\right)$, and where $\eta$ is some permutation of the set $\{1,2, \ldots, m+n\}$ (that is, a bijection from this set to itself) satisfying $\eta^{-1}(1)<\eta^{-1}(2)<\cdots<\eta^{-1}(m)$ (this causes the letters $\pi_{1}, \pi_{2}, \ldots, \pi_{m}$ to appear in the word $\left(\gamma_{\eta(1)}, \gamma_{\eta(2)}, \ldots, \gamma_{\eta(m+n)}\right)$ in this order $)$ and $\eta^{-1}(m+1)<\eta^{-1}(m+2)<\cdots<\eta^{-1}(m+n)$ (this causes the letters $\sigma_{1}, \sigma_{2}, \ldots, \sigma_{n}$ to appear in the word $\left(\gamma_{\eta(1)}, \gamma_{\eta(2)}, \ldots, \gamma_{\eta(m+n)}\right)$ in this order). Furthermore, the proper generalization of $S(\pi, \sigma)$ to this case would be a multiset, not a mere set.
} 
For example, $\left\{k^{2} \mid k \in\{-2,-1,0,1,2\}\right\}_{\text {multi }}$ is the multiset that contains the element 4 twice, the element 1 twice, and the element 0 once (and no other elements). This multiset can also be written in the form $\{4,1,0,1,4\}_{\text {multi }}$, or in the form $\{0,1,1,4,4\}_{\text {multi }}$.

Definition 12. Let st be a permutation statistic. We say that st is shuffle-compatible if and only if it has the following property: For any two disjoint permutations $\pi$ and $\sigma$, the multiset

$$
\{\text { st } \tau \mid \tau \in S(\pi, \sigma)\}_{\text {multi }}
$$

depends only on st $\pi$, st $\sigma,|\pi|$ and $|\sigma|$.

In other words, a permutation statistic st is shuffle-compatible if and only if it has the following property:

- If $\pi$ and $\sigma$ are two disjoint permutations, and if $\pi^{\prime}$ and $\sigma^{\prime}$ are two disjoint permutations, and if these permutations satisfy

$$
\begin{aligned}
\text { st } \pi & =\operatorname{st}\left(\pi^{\prime}\right), \quad \text { st } \sigma=\operatorname{st}\left(\sigma^{\prime}\right), \\
|\pi| & =\left|\pi^{\prime}\right| \quad \text { and } \quad|\sigma|=\left|\sigma^{\prime}\right|,
\end{aligned}
$$

then

$$
\{\text { st } \tau \mid \tau \in S(\pi, \sigma)\}_{\text {multi }}=\left\{\text { st } \tau \mid \tau \in S\left(\pi^{\prime}, \sigma^{\prime}\right)\right\}_{\text {multi }} .
$$

The notion of a shuffle-compatible permutation statistic was coined by Gessel and Zhuang in [4], where various statistics were analyzed for their shuffle-compatibility. In particular, it was shown in [4] that the statistics Des, Pk, Lpk and Rpk are shufflecompatible. Our next goal is to prove the same for the statistic Epk.

\section{Extending enriched $\boldsymbol{P}$-partitions and the exterior peak set}

We are going to define $\mathcal{Z}$-enriched $P$-partitions, which are a straightforward generalization of the notions of "P-partitions" [14], "enriched $P$-partitions" [16, §2] and "left enriched $P$-partitions" [11]. We will then consider a new particular case of this notion, which leads to a proof of the shuffle-compatibility of Epk conjectured in [4] (Theorem 60 below).

We remark that Bruce Sagan and Duff Baker-Jarvis are currently working on an alternative, bijective approach to the shuffle-compatibility of permutation statistics, which may lead to a different proof of this fact.

\subsection{Lacunar sets}

First, let us briefly study lacunar sets, a class of subsets of $\mathbb{Z}$ that are closely connected to exterior peaks. We start with the definition:

Definition 13. A set $S$ of integers is said to be lacunar if each $s \in S$ satisfies $s+1 \notin S$. 
In other words, a set of integers is lacunar if and only if it contains no two consecutive integers. For example, the set $\{2,5,7\}$ is lacunar, while the set $\{2,5,6\}$ is not.

Lacunar sets of integers are also called sparse sets in some of the literature (though the latter word has several competing meanings).

Definition 14. Let $n \in \mathbb{N}$. We define a set $\mathbf{L}_{n}$ of subsets of $[n]$ as follows:

- If $n$ is positive, then $\mathbf{L}_{n}$ shall mean the set of all nonempty lacunar subsets of $[n]$.

- If $n=0$, then $\mathbf{L}_{n}$ shall mean the set $\{\varnothing\}$.

For example,

$$
\begin{aligned}
& \mathbf{L}_{0}=\{\varnothing\} ; \quad \mathbf{L}_{1}=\{\{1\}\} ; \quad \mathbf{L}_{2}=\{\{1\},\{2\}\} ; \\
& \mathbf{L}_{3}=\{\{1\},\{2\},\{3\},\{1,3\}\} .
\end{aligned}
$$

Proposition 15. Let $\left(f_{0}, f_{1}, f_{2}, \ldots\right.$ ) be the Fibonacci sequence (defined by $f_{0}=0$ and $f_{1}=1$ and the recursive relation $f_{m}=f_{m-1}+f_{m-2}$ for all $\left.m \geqslant 2\right)$. Let $n$ be a positive integer. Then, $\left|\mathbf{L}_{n}\right|=f_{n+2}-1$.

Proof of Proposition 15. Recall that $\mathbf{L}_{n}$ is the set of all nonempty lacunar subsets of $[n]$ (since $n$ is positive). Thus, $\left|\mathbf{L}_{n}\right|$ is the number of all lacunar subsets of $[n]$ minus 1 (since the empty set $\varnothing$, which is clearly a lacunar subset of $[n]$, is withheld from the count). But a known fact (see, e.g., [15, Exercise 1.35 a.]) says that the number of lacunar subsets of $[n]$ is $f_{n+2}$. Combining the preceding two sentences, we conclude that $\left|\mathbf{L}_{n}\right|=f_{n+2}-1$. This proves Proposition 15.

The following observation is easy:

Proposition 16. Let $n \in \mathbb{N}$. Let $\pi$ be an n-permutation. Then, Epk $\pi \in \mathbf{L}_{n}$.

Proof of Proposition 16. If $n=0$, then the statement is obvious (since in this case, we have $\left.\operatorname{Epk} \pi=\varnothing \in \mathbf{L}_{0}\right)$. Thus, WLOG assume that $n \neq 0$. Hence, $n$ is positive. Hence, $\mathbf{L}_{n}$ is the set of all nonempty lacunar subsets of $[n]$ (by the definition of $\mathbf{L}_{n}$ ).

The set Epk $\pi$ is lacunar (since two consecutive integers cannot both be exterior peaks of $\pi$ ), and is also nonempty ( $\operatorname{since} \pi^{-1}(n)$ is an exterior peak of $\pi$ ). Therefore, Epk $\pi$ is a nonempty lacunar subset of $[n]$. In other words, Epk $\pi \in \mathbf{L}_{n}$ (since $\mathbf{L}_{n}$ is the set of all nonempty lacunar subsets of $[n])$. This proves Proposition 16.

Proposition 16 actually has a sort of converse:

Proposition 17. Let $n \in \mathbb{N}$. Let $\Lambda$ be a subset of $[n]$. Then, there exists an n-permutation $\pi$ satisfying $\Lambda=$ Epk $\pi$ if and only if $\Lambda \in \mathbf{L}_{n}$.

Proof of Proposition 17. Omitted; see [7] for a proof.

Next, let us introduce a total order on the finite subsets of $\mathbb{Z}$ : 
Definition 18. (a) Let $\mathbf{P}$ be the set of all finite subsets of $\mathbb{Z}$.

(b) If $A$ and $B$ are any two sets, then $A \triangle B$ shall denote the symmetric difference of $A$ and $B$. This is the set $(A \cup B) \backslash(A \cap B)=(A \backslash B) \cup(B \backslash A)$. It is well-known that the binary operation $\triangle$ on sets is associative.

If $A$ and $B$ are two distinct sets, then the set $A \triangle B$ is nonempty. Also, if $A \in \mathbf{P}$ and $B \in \mathbf{P}$, then $A \triangle B \in \mathbf{P}$. Thus, if $A$ and $B$ are two distinct sets in $\mathbf{P}$, then $\min (A \triangle B) \in \mathbb{Z}$ is well-defined.

(c) We define a binary relation $<$ on $\mathbf{P}$ as follows: For any $A \in \mathbf{P}$ and $B \in \mathbf{P}$, we let $A<B$ if and only if $A \neq B$ and $\min (A \triangle B) \in A$. (This definition makes sense, because the condition $A \neq B$ ensures that $\min (A \triangle B)$ is well-defined.)

Note that this relation $<$ is similar to the relation $<$ in [1, Lemma 4.3].

Proposition 19. The relation $<$ on $\mathbf{P}$ is the smaller relation of a total order on $\mathbf{P}$.

Proof of Proposition 19. See [7] for this straightforward argument (or imitate [1, proof of Lemma 4.3]).

In the following, we shall regard the set $\mathbf{P}$ as a totally ordered set, equipped with the order from Proposition 19. Thus, for example, two sets $A$ and $B$ in $\mathbf{P}$ satisfy $A \geqslant B$ if and only if either $A=B$ or $B<A$.

Definition 20. Let $S$ be a subset of $\mathbb{Z}$. Then, we define a new subset $S+1$ of $\mathbb{Z}$ by setting

$$
S+1=\{i+1 \mid i \in S\}=\{j \in \mathbb{Z} \mid j-1 \in S\} .
$$

Note that $S+1 \in \mathbf{P}$ if $S \in \mathbf{P}$.

For example, $\{2,5\}+1=\{3,6\}$. Note that a subset $S$ of $\mathbb{Z}$ is lacunar if and only if $S \cap(S+1)=\varnothing$.

Proposition 21. Let $\Lambda \in \mathbf{P}$ and $R \in \mathbf{P}$ be such that the set $R$ is lacunar and $R \subseteq$ $\Lambda \cup(\Lambda+1)$. Then, $R \geqslant \Lambda$ (with respect to the total order on $\mathbf{P}$ ).

Proof of Proposition 21. Assume the contrary. Thus, $R<\Lambda$ (since $\mathbf{P}$ is totally ordered). In other words, $R \neq \Lambda$ and $\min (R \triangle \Lambda) \in R$ (by the definition of the relation $<$ ). Let $\mu=\min (R \triangle \Lambda)$. Thus, $\mu=\min (R \triangle \Lambda) \in R \subseteq \Lambda \cup(\Lambda+1)$.

We have $\mu=\min (R \triangle \Lambda) \in R \triangle \Lambda=(R \cup \Lambda) \backslash(R \cap \Lambda)$. Hence, $\mu \notin R \cap \Lambda$. If we had $\mu \in \Lambda$, then we would have $\mu \in R \cap \Lambda$ (since $\mu \in R$ and $\mu \in \Lambda$ ), which would contradict $\mu \notin R \cap \Lambda$. Thus, we cannot have $\mu \in \Lambda$. Hence, $\mu \notin \Lambda$. Combining $\mu \in \Lambda \cup(\Lambda+1)$ with $\mu \notin \Lambda$, we obtain $\mu \in(\Lambda \cup(\Lambda+1)) \backslash \Lambda \subseteq \Lambda+1$. In other words, $\mu-1 \in \Lambda$.

Every $x \in R \triangle \Lambda$ satisfies $x \geqslant \min (R \triangle \Lambda)$. Hence, if we had $\mu-1 \in R \triangle \Lambda$, then we would have $\mu-1 \geqslant \min (R \triangle \Lambda)=\mu$, which would contradict $\mu-1<\mu$. Thus, we cannot have $\mu-1 \in R \triangle \Lambda$. Thus, $\mu-1 \notin R \triangle \Lambda$. Combining this with $\mu-1 \in \Lambda$, we obtain 
$\mu-1 \in \Lambda \backslash(R \triangle \Lambda)=R \cap \Lambda$ (since every two sets $X$ and $Y$ satisfy $Y \backslash(X \triangle Y)=X \cap Y)$. Thus, $\mu-1 \in R \cap \Lambda \subseteq R$.

But the set $R$ is lacunar. In other words, each $s \in R$ satisfies $s+1 \notin R$ (by the definition of "lacunar"). Applying this to $s=\mu-1$, we obtain $(\mu-1)+1 \notin R$ (since $\mu-1 \in R$ ). This contradicts $(\mu-1)+1=\mu \in R$. This contradiction shows that our assumption was wrong; hence, Proposition 21 is proven.

\section{$2.2 \quad \mathcal{Z}$-enriched $(P, \gamma)$-partitions}

Convention 22. By abuse of notation, we will often use the same notation for a poset $P=(X, \leqslant)$ and its ground set $X$ when there is no danger of confusion. In particular, if $x$ is some object, then " $x \in P$ " shall mean " $x \in X$ ".

Definition 23. A labeled poset means a pair $(P, \gamma)$ consisting of a finite poset $P=(X, \leqslant)$ and an injective map $\gamma: X \rightarrow A$ for some totally ordered set $A$. The injective map $\gamma$ is called the labeling of the labeled poset $(P, \gamma)$. The poset $P$ is called the ground poset of the labeled poset $(P, \gamma)$.

Convention 24. Let $\mathcal{N}$ be a totally ordered set, whose (strict) order relation will be denoted by $\prec$. Let + and - be two distinct symbols. Let $\mathcal{Z}$ be a subset of the set $\mathcal{N} \times\{+,-\}$. For each $q=(n, s) \in \mathcal{Z}$, we denote the element $n \in \mathcal{N}$ by $|q|$, and we call the element $s \in\{+,-\}$ the sign of $q$. If $n \in \mathcal{N}$, then we will denote the two elements $(n,+)$ and $(n,-)$ of $\mathcal{N} \times\{+,-\}$ by $+n$ and $-n$, respectively.

We equip the set $\mathcal{Z}$ with a total order, whose (strict) order relation $\prec$ is defined by

$$
(n, s) \prec\left(n^{\prime}, s^{\prime}\right) \text { if and only if either } n \prec n^{\prime} \text { or }\left(n=n^{\prime} \text { and } s=- \text { and } s^{\prime}=+\right) \text {. }
$$

Let Pow $\mathcal{N}$ be the ring of all formal power series over $\mathbb{Q}$ in the indeterminates $x_{n}$ for $n \in \mathcal{N}$.

We fix $\mathcal{N}$ and $\mathcal{Z}$ throughout Subsection 2.2. That is, any result in this subsection is tacitly understood to begin with "Let $\mathcal{N}$ be a totally ordered set, whose (strict) order relation will be denoted by $\prec$, and let $\mathcal{Z}$ be a subset of the set $\mathcal{N} \times\{+,-\}$ "; and the notations of this convention shall always be in place throughout this Subsection.

Whenever $\prec$ denotes some strict order, the corresponding weak order will be denoted by $\preccurlyeq$. (Thus, $a \preccurlyeq b$ means " $a \prec b$ or $a=b$ ".)

Definition 25. Let $(P, \gamma)$ be a labeled poset. A $\mathcal{Z}$-enriched $(P, \gamma)$-partition means a map $f: P \rightarrow \mathcal{Z}$ such that for all $x<y$ in $P$, the following conditions hold:

(i) We have $f(x) \preccurlyeq f(y)$.

(ii) If $f(x)=f(y)=+n$ for some $n \in \mathcal{N}$, then $\gamma(x)<\gamma(y)$.

(iii) If $f(x)=f(y)=-n$ for some $n \in \mathcal{N}$, then $\gamma(x)>\gamma(y)$.

(Of course, this concept depends on $\mathcal{N}$ and $\mathcal{Z}$, but these will always be clear from the context.) 
Example 26. Let $P$ be the poset with the following Hasse diagram:

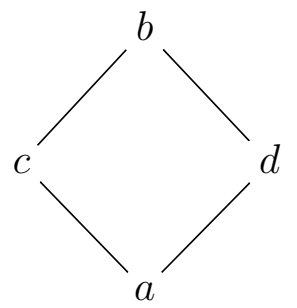

(that is, the ground set of $P$ is $\{a, b, c, d\}$, and its order relation is given by $a<c<b$ and $a<d<b)$. Let $\gamma: P \rightarrow \mathbb{Z}$ be a map that satisfies $\gamma(a)<\gamma(b)<\gamma(c)<\gamma(d)$ (for example, $\gamma$ could be the map that sends $a, b, c, d$ to $2,3,5,7$, respectively). Then, $(P, \gamma)$ is a labeled poset. A $\mathcal{Z}$-enriched $(P, \gamma)$-partition is a map $f: P \rightarrow \mathcal{Z}$ satisfying the following conditions:

(i) We have $f(a) \preccurlyeq f(c) \preccurlyeq f(b)$ and $f(a) \preccurlyeq f(d) \preccurlyeq f(b)$.

(ii) We cannot have $f(c)=f(b)=+n$ with $n \in \mathcal{N}$. We cannot have $f(d)=f(b)=+n$ with $n \in \mathcal{N}$.

(iii) We cannot have $f(a)=f(c)=-n$ with $n \in \mathcal{N}$. We cannot have $f(a)=f(d)=-n$ with $n \in \mathcal{N}$.

For example, if $\mathcal{N}=\mathbb{P}$ (the totally ordered set of positive integers, with its usual ordering) and $\mathcal{Z}=\mathcal{N} \times\{+,-\}$, then the map $f: P \rightarrow \mathcal{Z}$ sending $a, b, c, d$ to $+2,-3,+2,-3$ (respectively) is a $\mathcal{Z}$-enriched $(P, \gamma)$-partition. Notice that the total ordering on $\mathcal{Z}$ in this case is given by

$$
-1 \prec+1 \prec-2 \prec+2 \prec-3 \prec+3 \prec \cdots,
$$

rather than by the familiar total order on $\mathbb{Z}$.

The concept of a " $\mathcal{Z}$-enriched $(P, \gamma)$-partition" generalizes three notions in existing literature: that of a " $(P, \gamma)$-partition", that of an "enriched $(P, \gamma)$-partition", and that of a "left enriched $(P, \gamma)$-partition" 2

\footnotetext{
${ }^{2}$ The ideas behind these three concepts are due to Stanley [14], Stembridge [16, §2] and Petersen [11], respectively, but the precise definitions are not standardized across the literature. We define a " $(P, \gamma)$-partition" as in $[16, \S 1.1]$; this definition differs noticeably from Stanley's (in particular, Stanley requires $f(x) \succcurlyeq f(y)$ instead of $f(x) \preccurlyeq f(y)$, but the differences do not end here). We define an "enriched $(P, \gamma)$-partition" as in $[16, \S 2]$. Finally, we define a "left enriched $(P, \gamma)$-partition" to be a $\mathcal{Z}$-enriched $(P, \gamma)$-partition where $\mathcal{N}=\mathbb{N}$ and $\mathcal{Z}=(\mathcal{N} \times\{+,-\}) \backslash\{-0\}$; this definition is equivalent to Petersen's [12, Definition 3.4.1] up to some differences of notation (in particular, Petersen assumes that the ground set of $P$ is already a subset of $\mathbb{P}$, and that the labeling $\gamma$ is the canonical inclusion map $P \rightarrow \mathbb{P}$; also, he identifies the elements $+0,-1,+1,-2,+2, \ldots$ of $(\mathcal{N} \times\{+,-\}) \backslash\{-0\}$ with the integers $0,-1,+1,-2,+2, \ldots$, respectively). Note that the definition Petersen gives in [11, Definition 4.1] is incorrect, and the one in [12, Definition 3.4.1] is probably his intent.
} 


\section{Example 27.}

(a) If $\mathcal{N}=\mathbb{P}$ (the totally ordered set of positive integers) and $\mathcal{Z}=\mathcal{N} \times\{+\}=$ $\{+n \mid n \in \mathcal{N}\}$, then the $\mathcal{Z}$-enriched $(P, \gamma)$-partitions are simply the $(P, \gamma)$-partitions into $\mathcal{N}$, composed with the canonical bijection $\mathcal{N} \rightarrow \mathcal{Z}, n \mapsto(+n)$.

(b) If $\mathcal{N}=\mathbb{P}$ (the totally ordered set of positive integers) and $\mathcal{Z}=\mathcal{N} \times\{+,-\}$, then the $\mathcal{Z}$-enriched $(P, \gamma)$-partitions are the enriched $(P, \gamma)$-partitions.

(c) If $\mathcal{N}=\mathbb{N}$ (the totally ordered set of nonnegative integers) and $\mathcal{Z}=(\mathcal{N} \times\{+,-\}) \backslash$ $\{-0\}$, then the $\mathcal{Z}$-enriched $(P, \gamma)$-partitions are the left enriched $(P, \gamma)$-partitions. Note that +0 and -0 here stand for the pairs $(0,+)$ and $(0,-)$; thus, they are not equal.

Definition 28. If $(P, \gamma)$ is a labeled poset, then $\mathcal{E}(P, \gamma)$ shall denote the set of all $\mathcal{Z}$ enriched $(P, \gamma)$-partitions.

Definition 29. Let $P$ be any finite poset. Then, $\mathcal{L}(P)$ shall denote the set of all linear extensions of $P$. A linear extension of $P$ shall be understood simultaneously as a totally ordered set extending $P$ and as a list $\left(w_{1}, w_{2}, \ldots, w_{n}\right)$ of all elements of $P$ such that no two integers $i<j$ satisfy $w_{i} \geqslant w_{j}$ in $P$.

Let us prove some basic facts about $\mathcal{Z}$-enriched $(P, \gamma)$-partitions, straightforwardly generalizing classical results proven by Stanley and Gessel (for the case of "plain" $(P, \gamma)$ partitions), Stembridge [16, Lemma 2.1] (for enriched $(P, \gamma)$-partitions) and Petersen $[12$, Lemma 3.4.1] (for left enriched $(P, \gamma)$-partitions):

Proposition 30. For any labeled poset $(P, \gamma)$, we have

$$
\mathcal{E}(P, \gamma)=\bigsqcup_{w \in \mathcal{L}(P)} \mathcal{E}(w, \gamma)
$$

Proof of Proposition 30. This is analogous to the proof of [16, Lemma 2.1]. See [7] for details.

Definition 31. Let $(P, \gamma)$ be a labeled poset. We define a power series $\Gamma_{\mathcal{Z}}(P, \gamma) \in$ Pow $\mathcal{N}$ by

$$
\Gamma_{\mathcal{Z}}(P, \gamma)=\sum_{f \in \mathcal{E}(P, \gamma)} \prod_{p \in P} x_{|f(p)|}
$$

This is easily seen to be convergent in the usual topology on Pow $\mathcal{N}$. (Indeed, for every monomial $\mathfrak{m}$ in Pow $\mathcal{N}$, there exist at most $|P| ! \cdot 2^{|P|}$ many $f \in \mathcal{E}(P, \gamma)$ satisfying $\left.\prod_{p \in P} x_{|f(p)|}=\mathfrak{m}.\right)$

Corollary 32. For any labeled poset $(P, \gamma)$, we have

$$
\Gamma_{\mathcal{Z}}(P, \gamma)=\sum_{w \in \mathcal{L}(P)} \Gamma_{\mathcal{Z}}(w, \gamma)
$$


Proof of Corollary 32. Follows straight from Proposition 30.

Definition 33. Let $P$ be any set. Let $A$ be a totally ordered set. Let $\gamma: P \rightarrow A$ and $\delta: P \rightarrow A$ be two maps. We say that $\gamma$ and $\delta$ are order-isomorphic if the following holds: For every pair $(p, q) \in P \times P$, we have $\gamma(p) \leqslant \gamma(q)$ if and only if $\delta(p) \leqslant \delta(q)$.

Lemma 34. Let $(P, \alpha)$ and $(P, \beta)$ be two labeled posets with the same ground poset $P$. Assume that the maps $\alpha$ and $\beta$ are order-isomorphic. Then:

(a) We have $\mathcal{E}(P, \alpha)=\mathcal{E}(P, \beta)$.

(b) We have $\Gamma_{\mathcal{Z}}(P, \alpha)=\Gamma_{\mathcal{Z}}(P, \beta)$.

Proof of Lemma 34.

(a) If $x$ and $y$ are two elements of $P$, then we have the following equivalences:

$$
\begin{aligned}
& (\alpha(x) \leqslant \alpha(y)) \Longleftrightarrow(\beta(x) \leqslant \beta(y)) ; \\
& (\alpha(x)>\alpha(y)) \Longleftrightarrow(\beta(x)>\beta(y)) ; \\
& (\alpha(x)<\alpha(y)) \Longleftrightarrow(\beta(x)<\beta(y)) .
\end{aligned}
$$

(Indeed, the first of these equivalences holds because $\alpha$ and $\beta$ are order-isomorphic; the second is the contrapositive of the first; the third is obtained from the second by swapping $x$ with $y$.)

Hence, the conditions " $\alpha(x)>\alpha(y)$ " and " $\alpha(x)<\alpha(y)$ " in the definition of a $\mathcal{Z}$-enriched $(P, \alpha)$-partition are equivalent to the conditions " $\beta(x)>\beta(y)$ " and " $\beta(x)<\beta(y)$ " in the definition of a $\mathcal{Z}$-enriched $(P, \beta)$-partition. Therefore, the $\mathcal{Z}$-enriched $(P, \alpha)$-partitions are precisely the $\mathcal{Z}$-enriched $(P, \beta)$-partitions. In other words, $\mathcal{E}(P, \alpha)=\mathcal{E}(P, \beta)$. This proves Lemma 34 (a).

(b) Lemma 34 (b) follows from Lemma 34 (a).

Let us recall the notion of the disjoint union of two posets:

\section{Definition 35.}

(a) Let $P$ and $Q$ be two sets. The disjoint union of $P$ and $Q$ is the set $(\{0\} \times P) \cup$ $(\{1\} \times Q)$. This set is denoted by $P \sqcup Q$, and comes with two canonical injections

$$
\begin{array}{ll}
\iota_{0}: P \rightarrow P \sqcup Q, & p \mapsto(0, p), \quad \text { and } \\
\iota_{1}: Q \rightarrow P \sqcup Q, & q \mapsto(1, q) .
\end{array}
$$

The images of these two injections are disjoint, and their union is $P \sqcup Q$.

If $f: P \sqcup Q \rightarrow X$ is any map, then the restriction of $f$ to $P$ is understood to be the map $f \circ \iota_{0}: P \rightarrow X$, whereas the restriction of $f$ to $Q$ is understood to be the map $f \circ \iota_{1}: Q \rightarrow X$. (Of course, this notation is ambiguous when $P=Q$. ) 
When the sets $P$ and $Q$ are already disjoint, it is common to identify their disjoint union $P \sqcup Q$ with their union $P \cup Q$ via the map

$$
P \sqcup Q \rightarrow P \cup Q, \quad(i, r) \mapsto r
$$

Under this identification, the restriction of a map $f: P \sqcup Q \rightarrow X$ to $P$ becomes identical with the (literal) restriction $\left.f\right|_{P}$ of the map $f: P \cup Q \rightarrow X$ (and similarly for the restrictions to $Q$ ).

(b) Let $P$ and $Q$ be two posets. The disjoint union of the posets $P$ and $Q$ is the poset $P \sqcup Q$ whose ground set is the disjoint union $P \sqcup Q$, and whose order relation is defined by the following rules:

- If $p$ and $p^{\prime}$ are two elements of $P$, then $(0, p)<\left(0, p^{\prime}\right)$ in $P \sqcup Q$ if and only if $p<p^{\prime}$ in $P$.

- If $q$ and $q^{\prime}$ are two elements of $Q$, then $(1, q)<\left(1, q^{\prime}\right)$ in $P \sqcup Q$ if and only if $q<q^{\prime}$ in $Q$.

- If $p \in P$ and $q \in Q$, then the elements $(0, p)$ and $(1, q)$ of $P \sqcup Q$ are incomparable.

Proposition 36. Let $(P, \gamma)$ and $(Q, \delta)$ be two labeled posets. Let $(P \sqcup Q, \varepsilon)$ be a labeled poset whose ground poset $P \sqcup Q$ is the disjoint union of $P$ and $Q$, and whose labeling $\varepsilon$ is such that the restriction of $\varepsilon$ to $P$ is order-isomorphic to $\gamma$ and such that the restriction of $\varepsilon$ to $Q$ is order-isomorphic to $\delta$. Then,

$$
\Gamma_{\mathcal{Z}}(P, \gamma) \Gamma_{\mathcal{Z}}(Q, \delta)=\Gamma_{\mathcal{Z}}(P \sqcup Q, \varepsilon)
$$

Proof of Proposition 36. We WLOG assume that the ground sets $P$ and $Q$ are disjoint; thus, we can identify $P \sqcup Q$ with the union $P \cup Q$. Let us make this identification.

The restriction $\left.\varepsilon\right|_{P}$ of $\varepsilon$ to $P$ is order-isomorphic to $\gamma$. Hence, Lemma 34 (a) (applied to $\alpha=\left.\varepsilon\right|_{P}$ and $\left.\beta=\gamma\right)$ yields $\mathcal{E}\left(P,\left.\varepsilon\right|_{P}\right)=\mathcal{E}(P, \gamma)$. Similarly, $\mathcal{E}\left(Q,\left.\varepsilon\right|_{Q}\right)=\mathcal{E}(Q, \delta)$.

It is easy to see that a map $f: P \sqcup Q \rightarrow \mathcal{Z}$ is a $\mathcal{Z}$-enriched $(P \sqcup Q, \varepsilon)$-partition if and only if $\left.f\right|_{P}$ is a $\mathcal{Z}$-enriched $\left(P,\left.\varepsilon\right|_{P}\right)$-partition and $\left.f\right|_{Q}$ is a $\mathcal{Z}$-enriched $\left(Q,\left.\varepsilon\right|_{Q}\right)$-partition.

Therefore, the map

$$
\begin{aligned}
\mathcal{E}(P \sqcup Q, \varepsilon) & \rightarrow \mathcal{E}\left(P,\left.\varepsilon\right|_{P}\right) \times \mathcal{E}\left(Q,\left.\varepsilon\right|_{Q}\right), \\
f & \mapsto\left(\left.f\right|_{P},\left.f\right|_{Q}\right)
\end{aligned}
$$

is a bijection (this is easy to see). In other words, the map

$$
\begin{aligned}
\mathcal{E}(P \sqcup Q, \varepsilon) & \rightarrow \mathcal{E}(P, \gamma) \times \mathcal{E}(Q, \delta), \\
f & \mapsto\left(\left.f\right|_{P},\left.f\right|_{Q}\right)
\end{aligned}
$$


is a bijection $\left(\right.$ since $\mathcal{E}\left(P,\left.\varepsilon\right|_{P}\right)=\mathcal{E}(P, \gamma)$ and $\left.\mathcal{E}\left(Q,\left.\varepsilon\right|_{Q}\right)=\mathcal{E}(Q, \delta)\right)$. Now, the definition of $\Gamma_{\mathcal{Z}}(P \sqcup Q, \varepsilon)$ yields

$$
\begin{aligned}
& \begin{aligned}
& \Gamma_{\mathcal{Z}}(P \sqcup Q, \varepsilon)=\sum_{f \in \mathcal{E}(P \sqcup Q, \varepsilon)} \underbrace{\prod_{p \in P \sqcup Q} x_{|f(p)|}} \\
&=\left(\prod_{p \in P} x_{|f(p)|}\right)\left(\prod_{p \in Q} x_{|f(p)|}\right)
\end{aligned} \\
& =\sum_{f \in \mathcal{E}(P \sqcup Q, \mathcal{E})} \underbrace{\left(\prod_{p \in P} x_{|f(p)|}\right)}_{=\prod_{p \in P} x_{\left|\left(\left.f\right|_{P}\right)(p)\right|}} \underbrace{\left(\prod_{p \in Q} x_{|f(p)|}\right)}_{=\prod_{p \in Q} x\left|\left(\left.f\right|_{Q}\right)(p)\right|} \\
& =\sum_{f \in \mathcal{E}(P \sqcup Q, \varepsilon)}\left(\prod_{p \in P} x_{\left|\left(\left.f\right|_{P}\right)(p)\right|}\right)\left(\prod_{p \in Q} x_{\left|\left(\left.f\right|_{Q}\right)(p)\right|}\right) \\
& =\sum_{(g, h) \in \mathcal{E}(P, \gamma) \times \mathcal{E}(Q, \delta)}\left(\prod_{p \in P} x_{|g(p)|}\right)\left(\prod_{p \in Q} x_{|h(p)|}\right) \\
& \left(\begin{array}{c}
\text { here, we have substituted }(g, h) \text { for }\left(\left.f\right|_{P},\left.f\right|_{Q}\right), \\
\text { since the map }(1) \text { is a bijection }
\end{array}\right) \\
& =\underbrace{\left(\sum_{g \in \mathcal{E}(P, \gamma)} \prod_{p \in P} x_{|g(p)|}\right)}_{\sum_{f \in \mathcal{E}(P, \gamma)} \prod_{p \in P} x_{|f(p)|}=\Gamma \mathcal{Z}(P, \gamma)} \cdot \underbrace{\left(\sum_{h \in \mathcal{E}(Q, \delta)} \prod_{p \in Q} x_{|h(p)|}\right)}_{=\sum_{f \in \mathcal{E}(Q, \delta)} \prod_{p \in Q} x_{|f(p)|}=\Gamma \mathcal{Z}(Q, \delta)} \\
& =\Gamma_{\mathcal{Z}}(P, \gamma) \Gamma_{\mathcal{Z}}(Q, \delta) \text {. }
\end{aligned}
$$

This proves Proposition 36 .

Definition 37. Let $n \in \mathbb{N}$. Let $\pi$ be any $n$-permutation. (Recall that we have defined the concept of an " $n$-permutation" in Definition 3.) Then, $([n], \pi)$ is a labeled poset (in fact, $\pi$ is an injective map $[n] \rightarrow\{1,2,3, \ldots\}$, and thus can be considered a labeling). We define $\Gamma_{\mathcal{Z}}(\pi)$ to be the power series $\Gamma_{\mathcal{Z}}([n], \pi)$.

Let us recall the concept of a "poset homomorphism":

Definition 38. Let $P$ and $Q$ be two posets. A map $f: P \rightarrow Q$ is said to be a poset homomorphism if for any two elements $x$ and $y$ of $P$ satisfying $x \leqslant y$ in $P$, we have $f(x) \leqslant f(y)$ in $Q$.

It is well-known that if $U$ and $V$ are any two finite totally ordered sets of the same size, then there is a unique poset isomorphism $U \rightarrow V$. Thus, if $w$ is a finite totally ordered set with $n$ elements, then there is a unique poset isomorphism $w \rightarrow[n]$. Now, we claim the following: 
Proposition 39. Let $w$ be a finite totally ordered set with ground set $W$. Let $n=|W|$. Let $\bar{w}$ be the unique poset isomorphism $w \rightarrow[n]$. Let $\gamma: W \rightarrow\{1,2,3, \ldots\}$ be any injective map. Then, $\Gamma_{\mathcal{Z}}(w, \gamma)=\Gamma_{\mathcal{Z}}\left(\gamma \circ \bar{w}^{-1}\right)$.

Proof of Proposition 39. Clearly, $(w, \gamma)$ is a labeled poset (since $\gamma$ is injective). The map $\gamma \circ \bar{w}^{-1}:[n] \rightarrow\{1,2,3, \ldots\}$ is an injective map, thus an $n$-permutation. Hence, $\Gamma_{\mathcal{Z}}\left(\gamma \circ \bar{w}^{-1}\right)$ is well-defined, and its definition yields $\Gamma_{\mathcal{Z}}\left(\gamma \circ \bar{w}^{-1}\right)=\Gamma_{\mathcal{Z}}\left([n], \gamma \circ \bar{w}^{-1}\right)$. But $\bar{w}$ is a poset isomorphism $w \rightarrow[n]$, and thus is an isomorphism of labeled posets ${ }^{3}$ from $(w, \gamma)$ to $\left([n], \gamma \circ \bar{w}^{-1}\right)$. Hence,

$$
\begin{aligned}
\mathcal{E}(w, \gamma) & \rightarrow \mathcal{E}\left([n], \gamma \circ \bar{w}^{-1}\right), \\
f & \mapsto f \circ \bar{w}^{-1}
\end{aligned}
$$

is a bijection (since any isomorphism of labeled posets induces a bijection between their $\mathcal{Z}$-enriched $(P, \gamma)$-partitions). Furthermore, it satisfies $\prod_{p \in w} x_{|f(p)|}=\prod_{p \in[n]} x_{\left|\left(f \circ \bar{w}^{-1}\right)(p)\right|}$ for each $f \in \mathcal{E}(w, \gamma)$. Hence, $\Gamma_{\mathcal{Z}}(w, \gamma)=\Gamma_{\mathcal{Z}}\left([n], \gamma \circ \bar{w}^{-1}\right)=\Gamma_{\mathcal{Z}}\left(\gamma \circ \bar{w}^{-1}\right)$.

For the following corollary, let us recall that a bijective poset homomorphism is not necessarily an isomorphism of posets (since its inverse may and may not be a poset homomorphism).

Corollary 40. Let $(P, \gamma)$ be a labeled poset. Let $n=|P|$. Then,

$$
\Gamma_{\mathcal{Z}}(P, \gamma)=\sum_{\begin{array}{c}
x: P \rightarrow[n] \\
\text { bijective poset } \\
\text { homomorphism }
\end{array}} \Gamma_{\mathcal{Z}}\left(\gamma \circ x^{-1}\right) .
$$

Proof of Corollary 40. For each totally ordered set $w$ with ground set $P$, we let $\bar{w}$ be the unique poset isomorphism $w \rightarrow[n]$. If $w$ is a linear extension of $P$, then this map $\bar{w}$ is also a bijective poset homomorphism $P \rightarrow[n]$ (since every poset homomorphism $w \rightarrow[n]$ is also a poset homomorphism $P \rightarrow[n])$. Thus, for each $w \in \mathcal{L}(P)$, we have defined a bijective poset homomorphism $\bar{w}: P \rightarrow[n]$. We thus have defined a map

$$
\begin{aligned}
\mathcal{L}(P) & \rightarrow\{\text { bijective poset homomorphisms } P \rightarrow[n]\}, \\
w & \mapsto \bar{w} .
\end{aligned}
$$

This map is injective (indeed, a linear extension $w \in \mathcal{L}(P)$ can be uniquely reconstructed from $\bar{w}$ ) and surjective (because if $x$ is a bijective poset homomorphism $P \rightarrow[n]$, then the linear extension $w \in \mathcal{L}(P)$ defined (as a list) by $w=\left(x^{-1}(1), x^{-1}(2), \ldots, x^{-1}(n)\right)$ satisfies $x=\bar{w})$. Hence, this map is a bijection.

\footnotetext{
${ }^{3}$ We define the notion of an "isomorphism of labeled posets" in the obvious way: If $(P, \alpha)$ and $(Q, \beta)$ are two labeled posets, then a homomorphism of labeled posets from $(P, \alpha)$ to $(Q, \beta)$ means a poset homomorphism $f: P \rightarrow Q$ satisfying $\alpha=\beta \circ f$. A isomorphism of labeled posets is an invertible homomorphism of labeled posets whose inverse also is a homomorphism of labeled posets. Note that this definition of an isomorphism is not equivalent to the definition given in [16, Section 1.1].
} 
Corollary 32 yields

$$
\Gamma_{\mathcal{Z}}(P, \gamma)=\sum_{w \in \mathcal{L}(P)} \underbrace{\Gamma_{\mathcal{Z}}(w, \gamma)}_{\substack{=\Gamma_{\mathcal{Z}}\left(\gamma \circ \bar{w}^{-1}\right) \\ \text { (by Proposition 39) }}}=\sum_{w \in \mathcal{L}(P)} \Gamma_{\mathcal{Z}}\left(\gamma \circ \bar{w}^{-1}\right) .
$$

But recall that the map (2) is a bijection. Thus, we can substitute $x$ for $\bar{w}$ in the sum $\sum_{w \in \mathcal{L}(P)} \Gamma_{\mathcal{Z}}\left(\gamma \circ \bar{w}^{-1}\right)$, obtaining

$$
\sum_{w \in \mathcal{L}(P)} \Gamma_{\mathcal{Z}}\left(\gamma \circ \bar{w}^{-1}\right)=\sum_{\begin{array}{c}
x: P \rightarrow[n] \\
\text { bijective poset } \\
\text { homomorphism }
\end{array}} \Gamma_{\mathcal{Z}}\left(\gamma \circ x^{-1}\right)
$$

Hence, (3) becomes

$$
\Gamma_{\mathcal{Z}}(P, \gamma)=\sum_{w \in \mathcal{L}(P)} \Gamma_{\mathcal{Z}}\left(\gamma \circ \bar{w}^{-1}\right)=\sum_{\begin{array}{c}
x: P \rightarrow[n] \\
\text { bijective poset } \\
\text { homomorphism }
\end{array}} \Gamma_{\mathcal{Z}}\left(\gamma \circ x^{-1}\right)
$$

This proves Corollary 40.

Corollary 41. Let $n \in \mathbb{N}$ and $m \in \mathbb{N}$. Let $\pi$ be an n-permutation and let $\sigma$ be an $m$-permutation such that $\pi$ and $\sigma$ are disjoint. Then,

$$
\Gamma_{\mathcal{Z}}(\pi) \Gamma_{\mathcal{Z}}(\sigma)=\sum_{\tau \in S(\pi, \sigma)} \Gamma_{\mathcal{Z}}(\tau)
$$

Proof of Corollary 41. Consider the disjoint union $[n] \sqcup[m]$ of the posets $[n]$ and $[m]$. (Note that this disjoint union cannot be identified with the union $[n] \cup[m]$.) Let $\varepsilon$ be the map $[n] \sqcup[m] \rightarrow\{1,2,3, \ldots\}$ whose restriction to $[n]$ is $\pi$ and whose restriction to $[m]$ is $\sigma$. This map $\varepsilon$ is injective, since $\pi$ and $\sigma$ are disjoint permutations. Thus, $([n] \sqcup[m], \varepsilon)$ is a labeled poset.

The following two observations are easy to show (see [7] for detailed proofs):

Observation 1: If $x$ is a bijective poset homomorphism $[n] \sqcup[m] \rightarrow[n+m]$, then $\varepsilon \circ x^{-1} \in S(\pi, \sigma)$.

Observation 2: If $\tau \in S(\pi, \sigma)$, then there exists a unique bijective poset homomorphism $x:[n] \sqcup[m] \rightarrow[n+m]$ satisfying $\varepsilon \circ x^{-1}=\tau$.

Now, the map

$\{$ bijective poset homomorphisms $x:[n] \sqcup[m] \rightarrow[n+m]\} \rightarrow S(\pi, \sigma)$,

$$
x \mapsto \varepsilon \circ x^{-1}
$$


is well-defined (by Observation 1) and is a bijection (by Observation 2). Hence, we can substitute $\varepsilon \circ x^{-1}$ for $\tau$ in the sum $\sum_{\tau \in S(\pi, \sigma)} \Gamma_{\mathcal{Z}}(\tau)$. We thus obtain

$$
\sum_{\tau \in S(\pi, \sigma)} \Gamma_{\mathcal{Z}}(\tau)=\sum_{\begin{array}{c}
x:[n] \sqcup[m] \rightarrow[n+m] \\
\text { bijective poset } \\
\text { homomorphism }
\end{array}} \Gamma_{\mathcal{Z}}\left(\varepsilon \circ x^{-1}\right) .
$$

The definition of $\Gamma_{\mathcal{Z}}(\pi)$ yields $\Gamma_{\mathcal{Z}}(\pi)=\Gamma_{\mathcal{Z}}([n], \pi)$. The definition of $\Gamma_{\mathcal{Z}}(\sigma)$ yields $\Gamma_{\mathcal{Z}}(\sigma)=\Gamma_{\mathcal{Z}}([m], \sigma)$. Multiplying these two equalities, we obtain

$$
\begin{aligned}
\Gamma_{\mathcal{Z}}(\pi) \Gamma_{\mathcal{Z}}(\sigma)= & \Gamma_{\mathcal{Z}}([n], \pi) \Gamma_{\mathcal{Z}}([m], \sigma)=\Gamma_{\mathcal{Z}}([n] \sqcup[m], \varepsilon) \\
& \left(\begin{array}{c}
\text { by Proposition } 36, \text { applied to } P=[n], \gamma=\pi, \\
Q=[m] \text { and } \delta=\sigma
\end{array}\right) \\
= & \sum_{\substack{x:[n] \sqcup[m] \rightarrow[n+m] \\
\text { bijective poset } \\
\text { homomorphism }}} \Gamma_{\mathcal{Z}}\left(\varepsilon \circ x^{-1}\right) \\
= & \left(\begin{array}{c}
\text { by Corollary } 40, \text { applied to }[n] \sqcup[m], \varepsilon \\
\text { and } n+m \text { instead of } P, \gamma \text { and } n
\end{array}\right) \\
\sum_{\mathcal{Z}}(\tau) & \text { (by }(4)) .
\end{aligned}
$$

This proves Corollary 41.

\subsection{Exterior peaks}

So far we have been doing general nonsense. Let us now specialize to a situation that is connected to exterior peaks.

Convention 42. From now on, we set $\mathcal{N}=\{0,1,2, \ldots\} \cup\{\infty\}$, with total order given by $0 \prec 1 \prec 2 \prec \cdots \prec \infty$, and we set

$$
\begin{aligned}
\mathcal{Z} & =(\mathcal{N} \times\{+,-\}) \backslash\{-0,+\infty\} \\
& =\{+0\} \cup\{+n \mid n \in\{1,2,3, \ldots\}\} \cup\{-n \mid n \in\{1,2,3, \ldots\}\} \cup\{-\infty\} .
\end{aligned}
$$

Recall that the total order on $\mathcal{Z}$ has

$$
+0 \prec-1 \prec+1 \prec-2 \prec+2 \prec \cdots \prec-\infty .
$$

Definition 43. Let $S$ be a subset of $\mathbb{Z}$. A map $\chi$ from $S$ to a totally ordered set $K$ is said to be $V$-shaped if there exists some $t \in S$ such that the map $\left.\chi\right|_{\{s \in S \mid s \leqslant t\}}$ is strictly decreasing while the map $\left.\chi\right|_{\{s \in S \mid s \geqslant t\}}$ is strictly increasing. Notice that this $t \in S$ is uniquely determined in this case; namely, it is the unique $k \in S$ that minimizes $\chi(k)$. 
Thus, roughly speaking, a map from a subset of $\mathbb{Z}$ to a totally ordered set is $V$-shaped if and only if it is strictly decreasing up until a certain value of its argument, and then strictly increasing afterwards. For example, the 6-permutation $(5,1,2,3,4)$ is $\mathrm{V}$-shaped (keep in mind that we regard $n$-permutations as injective maps $[n] \rightarrow \mathbb{P}$ ), whereas the 4 -permutation $(3,1,4,2)$ is not.

Definition 44. Let $n \in \mathbb{N}$.

(a) Let $f:[n] \rightarrow \mathcal{Z}$ be any map. Then, $|f|$ shall denote the map $[n] \rightarrow \mathcal{N}, i \mapsto|f(i)|$.

(b) Let $g:[n] \rightarrow \mathcal{N}$ be any map. Then, we define a monomial $\mathbf{x}_{g}$ in Pow $\mathcal{N}$ by $\mathbf{x}_{g}=\prod_{i=1}^{n} x_{g(i)}$.

Using this definition, we can rewrite the definition of $\Gamma_{\mathcal{Z}}(\pi)$ as follows:

Proposition 45. Let $n \in \mathbb{N}$. Let $\pi$ be any $n$-permutation. Then,

$$
\Gamma_{\mathcal{Z}}(\pi)=\sum_{f \in \mathcal{E}([n], \pi)} \prod_{p \in[n]} x_{|f(p)|}=\sum_{f \in \mathcal{E}([n], \pi)} \mathbf{x}_{|f|} .
$$

Proof of Proposition 45. Easy consequence of the definitions (see [7] for details).

Definition 46. Let $n \in \mathbb{N}$. Let $g:[n] \rightarrow \mathcal{N}$ be any map. Let $\pi$ be an $n$-permutation. We shall say that $g$ is $\pi$-amenable if it has the following properties:

(i') The map $\left.\pi\right|_{g^{-1}(0)}$ is strictly increasing. (This allows the case when $g^{-1}(0)=\varnothing$.)

(ii') For each $h \in g([n]) \cap\{1,2,3, \ldots\}$, the map $\left.\pi\right|_{g^{-1}(h)}$ is V-shaped.

(iii') The map $\left.\pi\right|_{g^{-1}(\infty)}$ is strictly decreasing. (This allows the case when $g^{-1}(\infty)=\varnothing$.)

(iv') The map $g$ is weakly increasing.

Proposition 47. Let $n \in \mathbb{N}$. Let $\pi$ be any $n$-permutation. Then,

$$
\Gamma_{\mathcal{Z}}(\pi)=\sum_{\substack{g:[n] \rightarrow \mathcal{N} \\ \text { is } \pi \text {-amenable }}} 2^{|g([n]) \cap\{1,2,3, \ldots\}|} \mathbf{x}_{g} .
$$

Proof of Proposition 47 (sketched). The claim will immediately follow from (5) once we have shown the following two observations:

Observation 1: If $f \in \mathcal{E}([n], \pi)$, then the map $|f|:[n] \rightarrow \mathcal{N}$ is $\pi$-amenable.

Observation 2: If $g:[n] \rightarrow \mathcal{N}$ is a $\pi$-amenable map, then there exist precisely $2^{|g([n]) \cap\{1,2,3, \ldots\}|}$ maps $f \in \mathcal{E}([n], \pi)$ satisfying $|f|=g$. 
It thus remains to prove these two observations. Let us do this:

[Proof of Observation 1: Let $f \in \mathcal{E}([n], \pi)$. Thus, $f$ is a $\mathcal{Z}$-enriched $([n], \pi)$-partition. In other words, $f$ is a map $[n] \rightarrow \mathcal{Z}$ such that for all $x<y$ in $[n]$, the following conditions hold:

(i) We have $f(x) \preccurlyeq f(y)$.

(ii) If $f(x)=f(y)=+h$ for some $h \in \mathcal{N}$, then $\pi(x)<\pi(y)$.

(iii) If $f(x)=f(y)=-h$ for some $h \in \mathcal{N}$, then $\pi(x)>\pi(y)$.

(This is due to the definition of a $\mathcal{Z}$-enriched $([n], \pi)$-partition.)

Condition (i) shows that the map $f$ is weakly increasing. Condition (ii) shows that for each $h \in \mathcal{N}$, the map $\left.\pi\right|_{f^{-1}(+h)}$ is strictly increasing. Condition (iii) shows that for each $h \in \mathcal{N}$, the map $\left.\pi\right|_{f^{-1}(-h)}$ is strictly decreasing.

Now, set $g=|f|$. Then, $g^{-1}(0)=f^{-1}(+0)$ (since $\left.-0 \notin \mathcal{Z}\right)$. But the map $\left.\pi\right|_{f^{-1}(+0)}$ is strictly increasing ${ }^{4}$. Thus, the map $\left.\pi\right|_{g^{-1}(0)}$ is strictly increasing (since $g^{-1}(0)=$ $\left.f^{-1}(+0)\right)$. Hence, Property (i') in Definition 46 holds. Similarly, Property (iii') in that definition also holds.

Now, fix $h \in g([n]) \cap\{1,2,3, \ldots\}$. Then, the set $g^{-1}(h)$ is nonempty (since $h \in$ $g([n]))$, and can be written as the union of its two disjoint subsets $f^{-1}(+h)$ and $f^{-1}(-h)$. Furthermore, each element of $f^{-1}(-h)$ is smaller than each element of $f^{-1}(+h)$ (since $f$ is weakly increasing), and we know that the map $\left.\pi\right|_{f^{-1}(-h)}$ is strictly decreasing while the map $\left.\pi\right|_{f^{-1}(+h)}$ is strictly increasing. Hence, the map $\left.\pi\right|_{g^{-1}(h)}$ is strictly decreasing up until some value of its argument (namely, either the largest element of $f^{-1}(-h)$, or the smallest element of $f^{-1}(+h)$, depending on which of these two elements has the smaller image under $\pi$ ), and then strictly increasing from there on. In other words, the map $\left.\pi\right|_{g^{-1}(h)}$ is V-shaped. Thus, Property (ii') in Definition 46 holds. Finally, Property (iv') in Definition 46 holds because $f$ is weakly increasing. We have thus checked all four properties in Definition 46; thus, $g$ is $\pi$-amenable. In other words, $|f|$ is $\pi$-amenable (since $g=|f|$ ). This proves Observation 1.]

[Proof of Observation 2: Let $g:[n] \rightarrow \mathcal{N}$ be a $\pi$-amenable map. Consider a map $f \in \mathcal{E}([n], \pi)$ satisfying $|f|=g$. We are wondering to what extent the map $f$ is determined by $g$ and $\pi$.

Everything that we said in the proof of Observation 1 still holds in our situation (since $g=|f|)$.

In order to determine the map $f$, it clearly suffices to determine the sets $f^{-1}(q)$ for all $q \in \mathcal{Z}$. In other words, it suffices to determine the set $f^{-1}(+0)$, the set $f^{-1}(-\infty)$ and the sets $f^{-1}(+h)$ and $f^{-1}(-h)$ for all $h \in\{1,2,3, \ldots\}$.

Recall from the proof of Observation 1 that $g^{-1}(0)=f^{-1}(+0)$. Thus, $f^{-1}(+0)$ is uniquely determined by $g$. Similarly, $f^{-1}(-\infty)$ is uniquely determined by $g$. Thus, we can focus on the remaining sets $f^{-1}(+h)$ and $f^{-1}(-h)$ for $h \in\{1,2,3, \ldots\}$.

\footnotetext{
${ }^{4}$ because for each $h \in \mathcal{N}$, the map $\left.\pi\right|_{f^{-1}(+h)}$ is strictly increasing
} 
Fix $h \in\{1,2,3, \ldots\}$. Recall that the set $g^{-1}(h)$ is the union of its two disjoint subsets $f^{-1}(+h)$ and $f^{-1}(-h)$. Thus, $f^{-1}(+h)$ and $f^{-1}(-h)$ are complementary subsets of $g^{-1}(h)$. If $g^{-1}(h)=\varnothing$, then this uniquely determines $f^{-1}(+h)$ and $f^{-1}(-h)$. Thus, we focus only on the case when $g^{-1}(h) \neq \varnothing$.

So assume that $g^{-1}(h) \neq \varnothing$. Hence, $h \in g([n])$, so that $h \in g([n]) \cap\{1,2,3, \ldots\}$. Since the map $g$ is $\pi$-amenable, we thus conclude that the map $\left.\pi\right|_{g^{-1}(h)}$ is $V$-shaped (by Property (ii') in Definition 46).

The map $g$ is weakly increasing (by Property (iv') in Definition 46). Hence, $g^{-1}(h)$ is an interval of $[n]$. Let $\alpha \in \mathbb{Z}$ and $\gamma \in \mathbb{Z}$ be such that $g^{-1}(h)=[\alpha, \gamma]$ (where $[\alpha, \gamma]$ means the interval $\{\alpha, \alpha+1, \ldots, \gamma\})$.

As in the proof of Observation 1, we can see that each element of $f^{-1}(-h)$ is smaller than each element of $f^{-1}(+h)$. Since the union of $f^{-1}(-h)$ and $f^{-1}(+h)$ is $g^{-1}(h)=$ $[\alpha, \gamma]$, we thus conclude that (roughly speaking) the sets $f^{-1}(-h)$ and $f^{-1}(+h)$ partition the interval $g^{-1}(h)$ in two (possibly empty) sub-intervals such that the interval $f^{-1}(-h)$ lies completely to the left of $f^{-1}(+h)$. Hence, there exists some $\beta \in[\alpha-1, \gamma]$ such that $f^{-1}(-h)=[\alpha, \beta]$ and $f^{-1}(+h)=[\beta+1, \gamma]$. Consider this $\beta$. Clearly, $f^{-1}(-h)$ and $f^{-1}(+h)$ are uniquely determined by $\beta$; we just need to find out which values $\beta$ can take.

As in the proof of Observation 1, we can see that the map $\left.\pi\right|_{f^{-1}(-h)}$ is strictly decreasing while the map $\left.\pi\right|_{f^{-1}(+h)}$ is strictly increasing. Let $k$ be the element of $g^{-1}(h)$ minimizing $\pi(k)$. Then, the map $\pi$ is strictly decreasing on the set $\left\{u \in g^{-1}(h) \mid u \leqslant k\right\}$ and strictly increasing on the set $\left\{u \in g^{-1}(h) \mid u \geqslant k\right\}$ (since the map $\left.\pi\right|_{g^{-1}(h)}$ is V-shaped).

The map $\left.\pi\right|_{f^{-1}(-h)}$ is strictly decreasing. In other words, the map $\pi$ is strictly decreasing on the set $f^{-1}(-h)=[\alpha, \beta]$. On the other hand, the map $\pi$ is strictly increasing on the set $\left\{u \in g^{-1}(h) \mid u \geqslant k\right\}$. Hence, the two sets $[\alpha, \beta]$ and $\left\{u \in g^{-1}(h) \mid u \geqslant k\right\}$ cannot have more than one element in common (since $\pi$ is strictly decreasing on one and strictly increasing on the other). Thus, $k \geqslant \beta$. A similar argument shows that $k \leqslant \beta+1$. Combining these inequalities, we obtain $k \in\{\beta, \beta+1\}$, so that $\beta \in\{k, k-1\}$. This shows that $\beta$ can take only two values: $k$ and $k-1$.

Now, let us forget that we fixed $h$. We have shown that for each $h \in g([n]) \cap$ $\{1,2,3, \ldots\}$, the sets $f^{-1}(+h)$ and $f^{-1}(-h)$ are uniquely determined once the integer $\beta$ is chosen, and that this integer $\beta$ can be chosen in two ways. (As we have seen, all other values of $h$ do not matter.) Thus, in total, the map $f$ is uniquely determined up to $|g([n]) \cap\{1,2,3, \ldots\}|$ decisions, where each decision allows choosing from two values. Thus, there are at most $2^{|g([n]) \cap\{1,2,3, \ldots\}|}$ maps $f \in \mathcal{E}([n], \pi)$ satisfying $|f|=g$. Working the above argument backwards, we see that each way of making these decisions actually leads to a map $f \in \mathcal{E}([n], \pi)$ satisfying $|f|=g$; thus, there are exactly $2^{|g([n]) \cap\{1,2,3, \ldots\}|}$ maps $f \in \mathcal{E}([n], \pi)$ satisfying $|f|=g$. This proves Observation 2.]

Now, let us observe that if $g:[n] \rightarrow \mathcal{N}$ is a weakly increasing map (for some $n \in \mathbb{N}$ ), then the fibers of $g$ (that is, the subsets $g^{-1}(h)$ of $[n]$ for various $h \in \mathcal{N}$ ) are intervals of $[n]$ (possibly empty). Of course, when these fibers are nonempty, they have smallest elements and largest elements. We shall next study these elements more closely. 
Definition 48. Let $n \in \mathbb{N}$. Let $g:[n] \rightarrow \mathcal{N}$ be any map. We define a subset FE $(g)$ of $[n]$ as follows:

$$
\begin{aligned}
\mathrm{FE}(g)=\left\{\min \left(g^{-1}(h)\right) \mid h \in\{1,2,3, \ldots, \infty\} \text { with } g^{-1}(h) \neq \varnothing\right\} \\
\cup\left\{\max \left(g^{-1}(h)\right) \mid h \in\{0,1,2,3, \ldots\} \text { with } g^{-1}(h) \neq \varnothing\right\} .
\end{aligned}
$$

In other words, FE $(g)$ is the set comprising the smallest elements of all nonempty fibers of $g$ except for $g^{-1}(0)$ as well as the largest elements of all nonempty fibers of $g$ except for $g^{-1}(\infty)$. We shall refer to the elements of $\mathrm{FE}(g)$ as the fiber-ends of $g$.

Lemma 49. Let $n \in \mathbb{N}$. Let $\Lambda \in \mathbf{L}_{n}$. Then, there exists a weakly increasing map $g:[n] \rightarrow \mathcal{N}$ such that $\mathrm{FE}(g)=(\Lambda \cup(\Lambda+1)) \cap[n]$.

Proof of Lemma 49 (sketched). If $n=0$, then Lemma 49 holds for obvious reasons. Thus, WLOG assume that $n \neq 0$. Hence, $n$ is a positive integer. Thus, $\mathbf{L}_{n}$ is the set of all nonempty lacunar subsets of $[n]$ (by the definition of $\mathbf{L}_{n}$ ). Therefore, from $\Lambda \in \mathbf{L}_{n}$, we conclude that $\Lambda$ is a nonempty lacunar subset of $[n]$. Write this subset $\Lambda$ in the form $\Lambda=\left\{j_{1}<j_{2}<\cdots<j_{k}\right\}$. Thus, $k \geqslant 1$ (since $\Lambda$ is nonempty).

From $\left\{j_{1}<j_{2}<\cdots<j_{k}\right\}=\Lambda \subseteq[n]$, we obtain $0<j_{1}<j_{2}<\cdots<j_{k} \leqslant n$. Thus, $1<j_{1}+1<j_{2}+1<\cdots<j_{k}+1 \leqslant n+1$. Hence, the $k-1$ numbers $j_{1}+1, j_{2}+1, \ldots, j_{k-1}+1$ all belong to the set $[n]$, whereas the number $j_{k}+1$ only belongs to this set if $j_{k}<n$. Hence,

$$
\begin{aligned}
& \left\{j_{1}+1, j_{2}+1, \ldots, j_{k}+1\right\} \cap[n] \\
& =\left\{\begin{array}{ll}
\left\{j_{1}+1, j_{2}+1, \ldots, j_{k-1}+1, j_{k}+1\right\}, & \text { if } j_{k}<n ; \\
\left\{j_{1}+1, j_{2}+1, \ldots, j_{k-1}+1\right\}, & \text { if } j_{k}=n
\end{array} .\right.
\end{aligned}
$$

But $\Lambda=\left\{j_{1}<j_{2}<\cdots<j_{k}\right\}=\left\{j_{1}, j_{2}, \ldots, j_{k}\right\}$ and thus

$\Lambda+1=\left\{j_{1}+1, j_{2}+1, \ldots, j_{k}+1\right\}$. Hence,

$$
\begin{aligned}
(\Lambda+1) \cap[n] & =\left\{j_{1}+1, j_{2}+1, \ldots, j_{k}+1\right\} \cap[n] \\
& = \begin{cases}\left\{j_{1}+1, j_{2}+1, \ldots, j_{k-1}+1, j_{k}+1\right\}, & \text { if } j_{k}<n ; \\
\left\{j_{1}+1, j_{2}+1, \ldots, j_{k-1}+1\right\}, & \text { if } j_{k}=n\end{cases}
\end{aligned}
$$

Now, consider the map $g:[n] \rightarrow \mathcal{N}$ defined by

$$
\begin{gathered}
g(x)= \begin{cases}(\text { the number of all } \lambda \in \Lambda \text { such that } \lambda<x), & \text { if } x \leqslant j_{k} \\
\infty, & \text { if } x>j_{k}\end{cases} \\
\quad \text { for each } x \in[n] .
\end{gathered}
$$

Thus,

$$
(g(1), g(2), \ldots, g(n))
$$




$$
=(\underbrace{0,0, \ldots, 0}_{j_{1} \text { entries }}, \underbrace{1,1, \ldots, 1}_{j_{2}-j_{1} \text { entries }}, \underbrace{2,2, \ldots, 2}_{j_{3}-j_{2} \text { entries }}, \ldots, \underbrace{k-1, k-1, \ldots, k-1}_{j_{k}-j_{k-1} \text { entries }}, \underbrace{\infty, \infty, \ldots, \infty}_{n-j_{k} \text { entries }}) .
$$

The $n$-tuple on the right hand side of this equality consists of a block of 0's, followed by a block of 1's, followed by a block of 2's, and so on, all the way up to a block of $(k-1)$ 's, which is then followed by a block of $\infty$ 's. The first $k$ of these blocks are nonempty (since $0<j_{1}<j_{2}<\cdots<j_{k}$ ). The last block is nonempty if $j_{k}<n$, and empty if $j_{k}=n$. Thus, the map $g$ is weakly increasing, and its nonempty fibers are

$$
\begin{aligned}
g^{-1}(0) & =\left\{1,2, \ldots, j_{1}\right\}, \\
g^{-1}(1) & =\left\{j_{1}+1, j_{1}+2, \ldots, j_{2}\right\}, \\
g^{-1}(2) & =\left\{j_{2}+1, j_{2}+2, \ldots, j_{3}\right\}, \\
\vdots & \\
g^{-1}(k-1) & =\left\{j_{k-1}+1, j_{k-1}+2, \ldots, j_{k}\right\}, \\
g^{-1}(\infty) & =\left\{j_{k}+1, j_{k}+2, \ldots, n\right\},
\end{aligned}
$$

except that $g^{-1}(\infty)$ is empty when $j_{k}=n$.

Hence, the definition of $\mathrm{FE}(g)$ yields

$\mathrm{FE}(g)$

$$
\begin{aligned}
& =\underbrace{\left\{\min \left(g^{-1}(h)\right) \mid h \in\{1,2,3, \ldots, \infty\} \text { with } g^{-1}(h) \neq \varnothing\right\}} \\
& = \begin{cases}\left\{\min \left(g^{-1}(1)\right), \min \left(g^{-1}(2)\right), \ldots, \min \left(g^{-1}(k-1)\right), \min \left(g^{-1}(\infty)\right)\right\}, & \text { if } j_{k}<n ; \\
\left\{\min \left(g^{-1}(1)\right), \min \left(g^{-1}(2)\right), \ldots, \min \left(g^{-1}(k-1)\right)\right\}, & \text { if } j_{k}=n\end{cases} \\
& \cup \underbrace{\left\{\max \left(g^{-1}(h)\right) \mid h \in\left\{\left(g^{-1}(k-1)\right)\right\}\right.}_{=\left\{\max \left(g^{-1}(0)\right) \max \left(g^{-1}(1)\right), \max \left(g^{-1}(2)\right)\right.} \\
& =\underbrace{ \begin{cases}\left\{\min \left(g^{-1}(1)\right), \min \left(g^{-1}(2)\right), \ldots, \min \left(g^{-1}(k-1)\right), \min \left(g^{-1}(\infty)\right)\right\}, & \text { if } j_{k}<n ; \\
\left\{\min \left(g^{-1}(1)\right), \min \left(g^{-1}(2)\right), \ldots, \min \left(g^{-1}(k-1)\right)\right\}, & \text { if } j_{k}=n\end{cases} } \\
& = \begin{cases}\left\{j_{1}+1, j_{2}+1, \ldots, j_{k-1}+1, j_{k}+1\right\}, & \text { if } j_{k}<n ; \\
\left\{j_{1}+1, j_{2}+1, \ldots, j_{k-1}+1\right\}, & \text { if } j_{k}=n\end{cases} \\
& \text { (since } \min \left(g^{-1}(h)\right)=j_{h}+1 \text { for each } h \in\{1,2, \ldots, k-1\} \text {, } \\
& \text { and since } \left.\min \left(g^{-1}(\infty)\right)=j_{k}+1 \text { if } j_{k}<n\right) \\
& \cup \underbrace{\left\{\max \left(g^{-1}(0)\right), \max \left(g^{-1}(1)\right), \max \left(g^{-1}(2)\right), \ldots, \max \left(g^{-1}(k-1)\right)\right\}}_{=\left\{j_{1}, j_{2}, \ldots, j_{k}\right\}} \\
& \text { (since } \left.\max \left(g^{-1}(h-1)\right)=j_{h} \text { for each } h \in\{1,2, \ldots, k\}\right) \\
& =\underbrace{ \begin{cases}\left\{j_{1}+1, j_{2}+1, \ldots, j_{k-1}+1, j_{k}+1\right\}, & \text { if } j_{k}<n \\
\left\{j_{1}+1, j_{2}+1, \ldots, j_{k-1}+1\right\}, & \text { if } j_{k}=n\end{cases} }_{=\left(\begin{array}{c}
\Lambda+1) \cap[n] \\
\text { (by }(6))
\end{array}\right.} \cup \underbrace{\left\{j_{1}, j_{2}, \ldots, j_{k}\right\}}_{\begin{array}{c}
\{=\Lambda=\Lambda \cap[n] \\
(\text { since } \Lambda \subseteq[n])
\end{array}}
\end{aligned}
$$




$$
=((\Lambda+1) \cap[n]) \cup(\Lambda \cap[n])=(\Lambda \cap[n]) \cup((\Lambda+1) \cap[n])=(\Lambda \cup(\Lambda+1)) \cap[n] .
$$

Altogether, we have now shown that our map $g:[n] \rightarrow \mathcal{N}$ is weakly increasing and satisfies $\mathrm{FE}(g)=(\Lambda \cup(\Lambda+1)) \cap[n]$. Hence, such a map $g$ exists. Thus, Lemma 49 is proven.

Proposition 50. Let $n \in \mathbb{N}$. Let $\pi$ be an n-permutation. Let $g:[n] \rightarrow \mathcal{N}$ be any weakly increasing map. Then, the map $g$ is $\pi$-amenable if and only if $\operatorname{Epk} \pi \subseteq \mathrm{FE}(g)$.

Proof of Proposition 50. The map $g$ is weakly increasing. Thus, all nonempty fibers $g^{-1}(h)$ of $g$ are intervals of $[n]$. Recall that $g$ is $\pi$-amenable if and only if the four Properties (i'), (ii'), (iii') and (iv') in Definition 46 hold. Consider these four properties. Since Property (iv') automatically holds (since we assumed $g$ to be weakly increasing), we thus only need to discuss the other three:

- Property (i') is equivalent to the statement that every exterior peak of $\pi$ that lies in the fiber $g^{-1}(0)$ must be the largest element of this fiber. (Indeed, a strictly increasing map is characterized by having no exterior peaks except for the largest element of its domain.)

- Property (ii') is equivalent to the statement that every peak of $\pi$ that lies in a fiber $g^{-1}(h)$ with $h \in g([n]) \cap\{1,2,3, \ldots\}$ must be either the smallest or the largest element of this fiber (because the restriction $\left.\pi\right|_{g^{-1}(h)}$ is V-shaped if and only if no peak of $\pi$ appears in the interior of this fiber $\left.{ }^{5}\right)$. Moreover, we can replace the word "peak" by "exterior peak" in this sentence (since the exterior peaks 1 and $n$ must automatically be the smallest and the largest element of whatever fibers they belong to).

- Property (iii') is equivalent to the statement that every exterior peak of $\pi$ that lies in the fiber $g^{-1}(\infty)$ must be the smallest element of this fiber. (Indeed, a strictly decreasing map is characterized by having no exterior peaks except for the smallest element of its domain.)

Combining all of these insights, we conclude that the four Properties (i'), (ii'), (iii') and (iv') hold if and only if every exterior peak of $\pi$ is a fiber-end of $g$. In other words, $g$ is $\pi$-amenable if and only if every exterior peak of $\pi$ is a fiber-end of $g$ (since $g$ is $\pi$-amenable if and only if the four Properties (i'), (ii'), (iii') and (iv') hold). In other words, $g$ is $\pi$-amenable if and only if $\operatorname{Epk} \pi \subseteq \mathrm{FE}(g)$. This proves Proposition 50 .

We can rewrite Proposition 47 as follows, exhibiting its analogy with [16, Proposition $2.2]$ :

\footnotetext{
${ }^{5}$ The interior of an interval $\{a, a+1, \ldots, b\}$ of $[n]$ is defined to be the interval $\{a+1, a+2, \ldots, b-1\}$. (This is an empty interval if $a+1>b-1$.)
} 
Proposition 51. Let $n \in \mathbb{N}$. Let $\pi$ be any $n$-permutation. Then,

$$
\Gamma_{\mathcal{Z}}(\pi)=\sum_{\begin{array}{c}
g:[n] \rightarrow \mathcal{N} \text { is } \\
\text { weakly increasing; } \\
\text { Epk } \pi \subseteq \mathrm{FE}(g)
\end{array}} 2^{|g([n]) \cap\{1,2,3, \ldots\}|} \mathbf{x}_{g} .
$$

Proof of Proposition 51. Each $\pi$-amenable map $g:[n] \rightarrow \mathcal{N}$ is weakly increasing (because of Property (iv') in Definition 46). Hence, Proposition 50 yields that the $\pi$-amenable maps $g:[n] \rightarrow \mathcal{N}$ are precisely the weakly increasing maps $g:[n] \rightarrow \mathcal{N}$ satisfying Epk $\pi \subseteq \mathrm{FE}(g)$. Thus, Proposition 51 follows from Proposition 47.

Definition 52. Let $n \in \mathbb{N}$. If $\Lambda$ is any subset of $[n]$, then we define a power series $K_{n, \Lambda}^{\mathcal{Z}} \in \operatorname{Pow} \mathcal{N}$ by

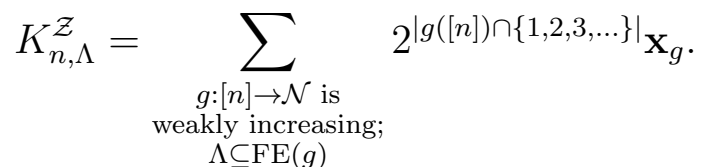

Thus, if $\pi$ is an $n$-permutation, then Proposition 51 shows that

$$
\Gamma_{\mathcal{Z}}(\pi)=K_{n, \operatorname{Epk} \pi}^{\mathcal{Z}}
$$

Remark 53. Let $n \in \mathbb{N}$. Let $\Lambda$ be any subset of $[n]$. It is easy to see that if $g:[n] \rightarrow \mathcal{N}$ is a weakly increasing map, and if $i \in[n]$, then $i \in \mathrm{FE}(g)$ holds if and only if we don't have $g(i-1)=g(i)=g(i+1)$, where we use the convention that $g(0)=0$ and $g(n+1)=\infty$. Hence, a weakly increasing map $g:[n] \rightarrow \mathcal{N}$ satisfies $\Lambda \subseteq \mathrm{FE}(g)$ if and only if no $i \in \Lambda$ satisfies $g(i-1)=g(i)=g(i+1)$, where we use the convention that $g(0)=0$ and $g(n+1)=\infty$. Thus, $(7)$ can be rewritten as follows:

$$
\begin{aligned}
K_{n, \Lambda}^{\mathcal{Z}}= & \sum_{\begin{array}{c}
g:[n] \rightarrow \mathcal{N} \text { is } \\
\text { weakly increasing; } \\
\text { no } i \in \Lambda \text { satisfies } g(i-1)=g(i)=g(i+1) \\
\text { (where we set } g(0)=0 \text { and } g(n+1)=\infty)
\end{array}} 2^{|g([n]) \cap\{1,2,3, \ldots\}|} \mathbf{x}_{g} \\
= & \sum_{\begin{array}{c}
\left(g_{1}, g_{2}, \ldots, g_{n}\right) \in \mathcal{N}^{n} ; \\
0 \preccurlyeq g_{1} \preccurlyeq g_{2} \preccurlyeq \cdots \preccurlyeq g_{n} \preccurlyeq \infty ; \\
\text { no } i \in \Lambda \text { satisfies } g_{i-1}=g_{i}=g_{i+1} \\
\text { (where we set } \left.g_{0}=0 \text { and } g_{n+1}=\infty\right)
\end{array}} 2^{\left|\left\{g_{1}, g_{2}, \ldots, g_{n}\right\} \cap\{1,2,3, \ldots\}\right|} x_{g_{1}} x_{g_{2}} \cdots x_{g_{n}}
\end{aligned}
$$

(here, we have substituted $\left(g_{1}, g_{2}, \ldots, g_{n}\right)$ for $(g(1), g(2), \ldots, g(n))$ in the sum). For example,

$$
\begin{aligned}
& K_{3,\{1,3\}}^{\mathcal{Z}}=\quad \sum^{\left|\left\{g_{1}, g_{2}, g_{3}\right\} \cap\{1,2,3, \ldots\}\right|} x_{g_{1}} x_{g_{2}} x_{g_{3}} \\
& \left.0 \preccurlyeq g_{1} \preccurlyeq g_{2} \preccurlyeq g_{3}\right) \in \mathcal{N}^{3} \text {; } ; \\
& \text { no } i \in\{1,3\} \text { satisfies } g_{i-1}=g_{i}=g_{i+1} \\
& \text { (where we set } g_{0}=0 \text { and } g_{4}=\infty \text { ) } \\
& =\sum_{\substack{\left(g_{1}, g_{2}, g_{3}\right) \in \mathcal{N}^{3} ; \\
0 \prec g_{1} \preccurlyeq g_{2} \prec g_{3} \preccurlyeq \infty ; \\
\text { neither } 0=g_{1}=g_{2} \text { nor } g_{2}=g_{3}=\infty \text { holds }}} 2^{\left|\left\{g_{1}, g_{2}, g_{3}\right\} \cap\{1,2,3, \ldots\}\right|} x_{g_{1}} x_{g_{2}} x_{g_{3}} .
\end{aligned}
$$


As a consequence of (9), we see that if we substitute 0 for $x_{0}$ and for $x_{\infty}$, then $K_{n, \Lambda}^{\mathcal{Z}}$ becomes the power series

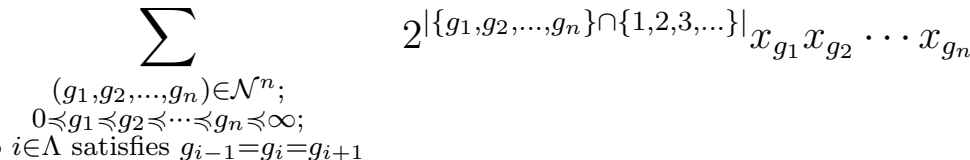

$$
\begin{aligned}
& \text { where we set } g_{0}=0 \text { and } g_{n+1}=\infty \text { ); } \\
& \text { none of the } g_{i} \text { equals } 0 \text { or } \\
& =\sum_{\substack{\left(g_{1}, g_{2}, \ldots, g_{n}\right) \in\{1,2,3, \ldots\}^{n} \\
g_{1} \preccurlyeq g_{2} \preccurlyeq \cdots \prec g_{n} ;}} 2^{\left|\left\{g_{1}, g_{2}, \ldots, g_{n}\right\}\right|} x_{g_{1}} x_{g_{2}} \cdots x_{g_{n}}
\end{aligned}
$$

in the indeterminates $x_{1}, x_{2}, x_{3}, \ldots$ This is called the "shifted quasi-symmetric function $\Theta_{\Lambda \backslash\{1, n\}}^{n}(X)$ " in $[2,(3.2)]$.

Corollary 41 now leads directly to the following multiplication rule (an analogue of $[16,(3.1)])$ :

Corollary 54. Let $n \in \mathbb{N}$ and $m \in \mathbb{N}$. Let $\pi$ be an n-permutation. Let $\sigma$ be an $m$ permutation such that $\pi$ and $\sigma$ are disjoint. Then,

$$
K_{n, \operatorname{Epk} \pi}^{\mathcal{Z}} \cdot K_{m, \operatorname{Epk} \sigma}^{\mathcal{Z}}=\sum_{\tau \in S(\pi, \sigma)} K_{n+m, \operatorname{Epk} \tau}^{\mathcal{Z}}
$$

Example 55. Applying Corollary 54 to $n=2, m=1, \pi=(1,2)$ and $\sigma=(3)$, we obtain

$$
K_{2, \operatorname{Epk}(1,2)}^{\mathcal{Z}} \cdot K_{1, \operatorname{Epk}(3)}^{\mathcal{Z}}=K_{3, \operatorname{Epk}(3,1,2)}^{\mathcal{Z}}+K_{3, \operatorname{Epk}(1,3,2)}^{\mathcal{Z}}+K_{3, \operatorname{Epk}(1,2,3)}^{\mathcal{Z}} \cdot
$$

In other words,

$$
K_{2,\{2\}}^{\mathcal{Z}} \cdot K_{1,\{1\}}^{\mathcal{Z}}=K_{3,\{1,3\}}^{\mathcal{Z}}+K_{3,\{2\}}^{\mathcal{Z}}+K_{3,\{3\}}^{\mathcal{Z}}
$$

Proof of Corollary 54. From (8), we obtain $\Gamma_{\mathcal{Z}}(\pi)=K_{n, \mathrm{Epk} \pi}^{\mathcal{Z}}$. Similarly, $\Gamma_{\mathcal{Z}}(\sigma)=K_{m, \operatorname{Epk} \sigma}^{\mathcal{Z}}$. Multiplying these two equalities, we obtain $\Gamma_{\mathcal{Z}}(\pi) \cdot \Gamma_{\mathcal{Z}}(\sigma)=K_{n, \operatorname{Epk} \pi}^{\mathcal{Z}}$. $K_{m, \operatorname{Epk} \sigma}^{\mathcal{Z}}$. Hence,

$$
\begin{aligned}
& K_{n, \mathrm{Epk} \pi}^{\mathcal{Z}} \cdot K_{m, \operatorname{Epk} \sigma}^{\mathcal{Z}}=\Gamma_{\mathcal{Z}}(\pi) \cdot \Gamma_{\mathcal{Z}}(\sigma)=\sum_{\tau \in S(\pi, \sigma)} \underbrace{\Gamma_{\mathcal{Z}}(\tau)}_{\begin{array}{c}
K_{n+m, \mathrm{Epk}}^{\mathcal{Z}} \\
(\mathrm{by}(8))
\end{array}} \\
& =\sum_{\tau \in S(\pi, \sigma)} K_{n+m, \operatorname{Epk} \tau}^{\mathcal{Z}} .
\end{aligned}
$$

This proves Corollary 54.

Recall Definition 14.

Proposition 56. Let $n \in \mathbb{N}$. Then, the family

$$
\left(K_{n, \Lambda}^{\mathcal{Z}}\right)_{\Lambda \in \mathbf{L}_{n}}
$$

is $\mathbb{Q}$-linearly independent. 
Our proof of Proposition 56 requires the following definition:

Definition 57. Let $\mathfrak{m}$ be any monomial in Pow $\mathcal{N}$ (that is, a formal commutative product of indeterminates $x_{h}$ with $\left.h \in \mathcal{N}\right)$. Let $f \in \operatorname{Pow} \mathcal{N}$. Then, $[\mathfrak{m}](f)$ shall mean the coefficient of $\mathfrak{m}$ in the power series $f$. (For example, $\left[x_{0}^{2} x_{3}\right]\left(3+5 x_{0}^{2} x_{3}+6 x_{0}+9 x_{\infty}\right)=5$ and $\left[x_{0}^{2} x_{3}\right]\left(x_{1}-x_{\infty}\right)=0$.)

Lemma 58. Let $n \in \mathbb{N}$.

(a) If $g$ and $h$ are two weakly increasing maps $[n] \rightarrow \mathcal{N}$, then

$$
\text { (we have } \mathbf{x}_{g}=\mathbf{x}_{h} \text { if and only if } g=h \text { ). }
$$

(b) Let $R \in \mathbf{L}_{n}$. Let $h:[n] \rightarrow \mathcal{N}$ be a weakly increasing map. Then,

$$
\left[\mathbf{x}_{h}\right]\left(K_{n, R}^{\mathcal{Z}}\right)= \begin{cases}2^{|h([n]) \cap\{1,2,3, \ldots\}|}, & \text { if } R \subseteq \mathrm{FE}(h) \\ 0, & \text { otherwise }\end{cases}
$$

Proof of Lemma 58. (a) A weakly increasing map $g:[n] \rightarrow \mathcal{N}$ can be uniquely reconstructed from the multiset $\{g(1), g(2), \ldots, g(n)\}_{\text {multi }}$ of its values (because it is weakly increasing, so there is only one way in which these values can be ordered). Hence, a weakly increasing map $g:[n] \rightarrow \mathcal{N}$ can be uniquely reconstructed from the monomial $\mathbf{x}_{g}$ (since this monomial $\mathbf{x}_{g}=x_{g(1)} x_{g(2)} \cdots x_{g(n)}$ encodes the multiset $\left.\{g(1), g(2), \ldots, g(n)\}_{\text {multi }}\right)$. In other words, if $g$ and $h$ are two weakly increasing maps $[n] \rightarrow \mathcal{N}$, then $\mathbf{x}_{g}=\mathbf{x}_{h}$ holds if and only if $g=h$. This proves Lemma 58 (a).

(b) The definition of $K_{n, R}^{\mathcal{Z}}$ yields

$$
K_{n, R}^{\mathcal{Z}}=\sum_{\substack{g:[n] \rightarrow \mathcal{N} \text { is } \\ \text { weakly increasing; } \\ R \subseteq \mathrm{FE}(g)}} 2^{|g([n]) \cap\{1,2,3, \ldots\}|} \mathbf{x}_{g} .
$$

Thus,

$$
\begin{aligned}
& {\left[\mathbf{x}_{h}\right]\left(K_{n, R}^{\mathcal{Z}}\right)=\left[\mathbf{x}_{h}\right]\left(\sum_{\substack{g:[n] \rightarrow \mathcal{N} \text { is } \\
\text { weakly increasing; } \\
R \subseteq \mathrm{FE}(g)}} 2^{|g([n]) \cap\{1,2,3, \ldots\}|} \mathbf{x}_{g}\right)} \\
& =\sum_{\substack{g:[n] \rightarrow \mathcal{N} \text { is } \\
\text { weakly increasing; } \\
R \subseteq \mathrm{FE}(g)}} 2^{|g([n]) \cap\{1,2,3, \ldots\}|} \\
& \underbrace{\left[\mathbf{x}_{h}\right]\left(\mathbf{x}_{g}\right)} \\
& = \begin{cases}1, & \text { if } \mathbf{x}_{g}=\mathbf{x}_{h} ; \\
0, & \text { if } \mathbf{x}_{g} \neq \mathbf{x}_{h}\end{cases} \\
& \text { (since } \mathbf{x}_{h} \text { and } \mathbf{x}_{g} \text { are two monomials) }
\end{aligned}
$$




$$
\begin{aligned}
& \begin{aligned}
&=\sum_{\substack{g:[n] \rightarrow \mathcal{N} \text { is } \\
\text { weakli increasing; } \\
R \subseteq \mathrm{FE}(g)}} 2^{|g([n]) \cap\{1,2,3, \ldots\}|} \underbrace{ \begin{cases}1, & \text { if } \mathbf{x}_{g}=\mathbf{x}_{h} ; \\
0, & \text { if } \mathbf{x}_{g} \neq \mathbf{x}_{h}\end{cases} } \\
&= \begin{cases}1, & \text { if } g=h ; \\
0, & \text { if } g \neq h \\
\text { (by Lemma } 58 \text { (a)) }\end{cases}
\end{aligned} \\
& =\sum_{\substack{g:[n] \rightarrow \mathcal{N} \text { is } \\
\text { weakly increasing; } \\
R \subseteq \mathrm{FE}(g)}} 2^{|g([n]) \cap\{1,2,3, \ldots\}|} \begin{cases}1, & \text { if } g=h ; \\
0, & \text { if } g \neq h\end{cases} \\
& =\sum_{\substack{g:[n] \rightarrow \mathcal{N} \text { is } \\
\text { weakly increasing; } \\
R \subseteq \mathrm{FE}(g) ; \\
g=h}} 2^{|g([n]) \cap\{1,2,3, \ldots\}|} .
\end{aligned}
$$

The sum on the right hand side of this equality has a unique addend (namely, its addend for $g=h$, which is $2^{|h([n]) \cap\{1,2,3, \ldots\}|}$ ) when $R \subseteq \mathrm{FE}(h)$; otherwise it is an empty sum. Hence, this sum simplifies as follows:

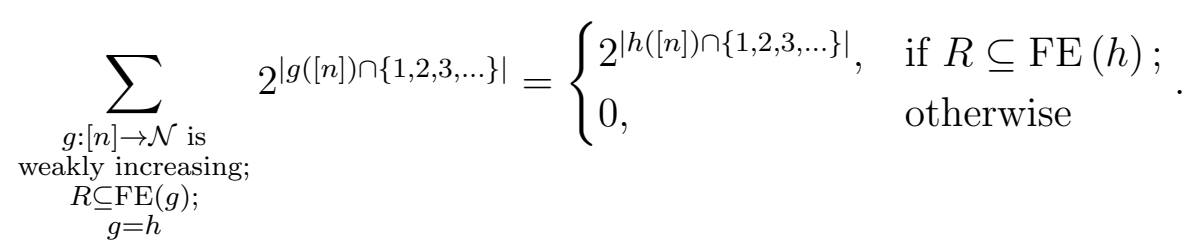

Hence,

$$
\left[\mathbf{x}_{h}\right]\left(K_{n, R}^{\mathcal{Z}}\right)=\sum_{\substack{g:[n] \rightarrow \mathcal{N} \text { is } \\
\text { weakly increasing; } \\
R \subseteq \mathrm{FE}(g) ; \\
g=h}} 2^{|g([n]) \cap\{1,2,3, \ldots\}|}=\left\{\begin{array}{ll}
2^{|h([n]) \cap\{1,2,3, \ldots\}|}, & \text { if } R \subseteq \mathrm{FE}(h) ; \\
0, & \text { otherwise }
\end{array} .\right.
$$

This proves Lemma 58 (b).

First proof of Proposition 56. Recall Definition 18. In the following, we shall regard the set $\mathbf{P}$ as a totally ordered set, equipped with the order from Proposition 19.

Clearly, $\mathbf{L}_{n} \subseteq \mathbf{P}$. Hence, we consider $\mathbf{L}_{n}$ as a totally ordered set, whose total order is inherited from $\mathbf{P}$.

Let $\left(a_{R}\right)_{R \in \mathbf{L}_{n}} \in \mathbb{Q}^{\mathbf{L}_{n}}$ be a family of scalars (in $\mathbb{Q}$ ) such that $\sum_{R \in \mathbf{L}_{n}} a_{R} K_{n, R}^{\mathcal{Z}}=0$. We are going to show that $\left(a_{R}\right)_{R \in \mathbf{L}_{n}}=(0)_{R \in \mathbf{L}_{n}}$.

Indeed, assume the contrary. Thus, $\left(a_{R}\right)_{R \in \mathbf{L}_{n}} \neq(0)_{R \in \mathbf{L}_{n}}$. Hence, there exists some $R \in \mathbf{L}_{n}$ such that $a_{R} \neq 0$. Let $\Lambda$ be the largest such $R$ (with respect to the total order on $\mathbf{L}_{n}$ we have introduced above). Hence, $\Lambda$ is an element of $\mathbf{L}_{n}$ and satisfies $a_{\Lambda} \neq 0$; but every element $R \in \mathbf{L}_{n}$ satisfying $R>\Lambda$ must satisfy

$$
a_{R}=0 .
$$


Lemma 49 shows that there exists a weakly increasing map $g:[n] \rightarrow \mathcal{N}$ such that $\operatorname{FE}(g)=(\Lambda \cup(\Lambda+1)) \cap[n]$. Consider this $g$. Combining $\Lambda \subseteq \Lambda \cup(\Lambda+1)$ with $\Lambda \subseteq[n]$, we obtain

$$
\Lambda \subseteq(\Lambda \cup(\Lambda+1)) \cap[n]=\mathrm{FE}(g) .
$$

For every $R \in \mathbf{L}_{n}$ satisfying $R \neq \Lambda$, we have

$$
\left[\mathbf{x}_{g}\right]\left(a_{R} K_{n, R}^{\mathcal{Z}}\right)=0 .
$$

[Proof of (11): Let $R \in \mathbf{L}_{n}$ be such that $R \neq \Lambda$. We must prove (11).

Assume the contrary. Thus, $\left[\mathbf{x}_{g}\right]\left(a_{R} K_{n, R}^{\mathcal{Z}}\right) \neq 0$. In other words, $a_{R}\left[\mathbf{x}_{g}\right]\left(K_{n, R}^{\mathcal{Z}}\right) \neq 0$. Hence, $a_{R} \neq 0$ and $\left[\mathbf{x}_{g}\right]\left(K_{n, R}^{\mathcal{Z}}\right) \neq 0$.

From the definition of $\mathbf{L}_{n}$, it follows easily that every element of $\mathbf{L}_{n}$ is a lacunar subset of $[n]$. Hence, $R$ is a lacunar subset of $[n]$ (since $R \in \mathbf{L}_{n}$ ).

But Lemma 58 (b) (applied to $h=g$ ) yields

$$
\left[\mathbf{x}_{g}\right]\left(K_{n, R}^{\mathcal{Z}}\right)= \begin{cases}2^{|g([n]) \cap\{1,2,3, \ldots\}|}, & \text { if } R \subseteq \mathrm{FE}(g) ; \\ 0, & \text { otherwise }\end{cases}
$$

Hence, $\left[\mathbf{x}_{g}\right]\left(K_{n, R}^{\mathcal{Z}}\right)=0$ if $R \nsubseteq \mathrm{FE}(g)$. Thus, we cannot have $R \nsubseteq \mathrm{FE}(g)$ (since $\left.\left[\mathbf{x}_{g}\right]\left(K_{n, R}^{\mathcal{Z}}\right) \neq 0\right)$. Therefore, we have

$$
R \subseteq \mathrm{FE}(g)=(\Lambda \cup(\Lambda+1)) \cap[n] \subseteq \Lambda \cup(\Lambda+1) .
$$

Thus, Proposition 21 yields that $R \geqslant \Lambda$. Combining this with $R \neq \Lambda$, we obtain $R>\Lambda$. Hence, (10) yields $a_{R}=0$. This contradicts $a_{R} \neq 0$. This contradiction shows that our assumption was wrong. Hence, (11) is proven.]

On the other hand, Lemma 58 (b) (applied to $h=g$ and $R=\Lambda$ ) yields

$$
\left[\mathbf{x}_{g}\right]\left(K_{n, \Lambda}^{\mathcal{Z}}\right)= \begin{cases}2^{|g([n]) \cap\{1,2,3, \ldots\}|}, & \text { if } \Lambda \subseteq \mathrm{FE}(g) ;=2^{|g([n]) \cap\{1,2,3, \ldots\}|} \\ 0, & \text { otherwise }\end{cases}
$$

(since $\Lambda \subseteq \mathrm{FE}(g)$ ). fore,

Now, recall that $\sum_{R \in \mathbf{L}_{n}} a_{R} K_{n, R}^{\mathcal{Z}}=0$. Hence, $\left[\mathbf{x}_{g}\right]\left(\sum_{R \in \mathbf{L}_{n}} a_{R} K_{n, R}^{\mathcal{Z}}\right)=\left[\mathbf{x}_{g}\right](0)=0$. There-

$$
\begin{aligned}
0 & =\left[\mathbf{x}_{g}\right]\left(\sum_{R \in \mathbf{L}_{n}} a_{R} K_{n, R}^{\mathcal{Z}}\right)=\sum_{R \in \mathbf{L}_{n}}\left[\mathbf{x}_{g}\right]\left(a_{R} K_{n, R}^{\mathcal{Z}}\right) \\
& =\left[\mathbf{x}_{g}\right]\left(a_{\Lambda} K_{n, \Lambda}^{\mathcal{Z}}\right)+\sum_{\substack{R \in \mathbf{L}_{n} ; \\
R \neq \Lambda}}^{\left[\mathbf{x}_{g}\right]\left(a_{R} K_{n, R}^{\mathcal{Z}}\right)} \quad\left(\text { since } \Lambda \in \mathbf{L}_{n}\right) \\
& =\left[\mathbf{x}_{g}\right]\left(a_{\Lambda} K_{n, \Lambda}^{\mathcal{Z}}\right)=a_{\Lambda} \underbrace{\left[\mathbf{x}_{g}\right]\left(K_{n, \Lambda}^{\mathcal{Z}}\right)}_{=2^{\mid g([n]) \cap(11))}}=\underbrace{a_{\Lambda}}_{\neq 0} \underbrace{2^{|g([n]) \cap\{1,2,3, \ldots\}|}}_{\neq 0} \neq 0 .,
\end{aligned}
$$


This contradiction shows that our assumption was false. Hence, $\left(a_{R}\right)_{R \in \mathbf{L}_{n}}=(0)_{R \in \mathbf{L}_{n}}$ is proven.

Now, forget that we fixed $\left(a_{R}\right)_{R \in \mathbf{L}_{n}}$. We thus have shown that if $\left(a_{R}\right)_{R \in \mathbf{L}_{n}} \in \mathbb{Q}^{\mathbf{L}_{n}}$ is a family of scalars (in $\mathbb{Q}$ ) such that $\sum_{R \in \mathbf{L}_{n}} a_{R} K_{n, R}^{\mathcal{Z}}=0$, then $\left(a_{R}\right)_{R \in \mathbf{L}_{n}}=(0)_{R \in \mathbf{L}_{n}}$. In other words, the family $\left(K_{n, R}^{\mathcal{Z}}\right)_{R \in \mathbf{L}_{n}}$ is $\mathbb{Q}$-linearly independent. In other words, the family $\left(K_{n, \Lambda}^{\mathcal{Z}}\right)_{\Lambda \in \mathbf{L}_{n}}$ is $\mathbb{Q}$-linearly independent. This proves Proposition 56.

A second proof of Proposition 56 can be found in [7].

Corollary 59. The family

$$
\left(K_{n, \Lambda}^{\mathcal{Z}}\right)_{n \in \mathbb{N} ; \Lambda \in \mathbf{L}_{n}}
$$

is $\mathbb{Q}$-linearly independent.

Proof of Corollary 59. Follows from Proposition 56 using gradedness; see [7] for details.

We can now finally prove what we came here for:

Theorem 60. The permutation statistic Epk is shuffle-compatible.

Proof of Theorem 60. We must prove that Epk is shuffle-compatible. In other words, we must prove that for any two disjoint permutations $\pi$ and $\sigma$, the multiset

$\{\operatorname{Epk} \tau \mid \tau \in S(\pi, \sigma)\}_{\text {multi }}$ depends only on $\operatorname{Epk} \pi, \operatorname{Epk} \sigma,|\pi|$ and $|\sigma|$. In other words, we must prove that if $\pi$ and $\sigma$ are two disjoint permutations, and if $\pi^{\prime}$ and $\sigma^{\prime}$ are two disjoint permutations satisfying $\operatorname{Epk} \pi=\operatorname{Epk}\left(\pi^{\prime}\right), \operatorname{Epk} \sigma=\operatorname{Epk}\left(\sigma^{\prime}\right),|\pi|=\left|\pi^{\prime}\right|$ and $|\sigma|=\left|\sigma^{\prime}\right|$, then the multiset $\{\operatorname{Epk} \tau \mid \tau \in S(\pi, \sigma)\}_{\text {multi }}$ equals the multiset $\left\{\operatorname{Epk} \tau \mid \tau \in S\left(\pi^{\prime}, \sigma^{\prime}\right)\right\}_{\text {multi }}$.

So let $\pi$ and $\sigma$ be two disjoint permutations, and let $\pi^{\prime}$ and $\sigma^{\prime}$ be two disjoint permutations satisfying $\operatorname{Epk} \pi=\operatorname{Epk}\left(\pi^{\prime}\right), \operatorname{Epk} \sigma=\operatorname{Epk}\left(\sigma^{\prime}\right),|\pi|=\left|\pi^{\prime}\right|$ and $|\sigma|=\left|\sigma^{\prime}\right|$.

Define $n \in \mathbb{N}$ by $n=|\pi|=\left|\pi^{\prime}\right|$ (this is well-defined, since $\left.|\pi|=\left|\pi^{\prime}\right|\right)$. Likewise, define $m \in \mathbb{N}$ by $m=|\sigma|=\left|\sigma^{\prime}\right|$. Thus, $\pi$ is an $n$-permutation, while $\sigma$ is an $m$-permutation. Hence, each $\tau \in S(\pi, \sigma)$ is an $(n+m)$-permutation, and therefore satisfies $\operatorname{Epk} \tau \in \mathbf{L}_{n+m}$ (by Proposition 16, applied to $n+m$ and $\tau$ instead of $n$ and $\pi$ ). Thus, the multiset $\{\operatorname{Epk} \tau \mid \tau \in S(\pi, \sigma)\}_{\text {multi }}$ consists of elements of $\mathbf{L}_{n+m}$. The same holds for the multiset $\left\{\operatorname{Epk} \tau \mid \tau \in S\left(\pi^{\prime}, \sigma^{\prime}\right)\right\}_{\text {multi }}$ (for similar reasons).

Corollary 54 yields

$$
\begin{aligned}
& K_{n, \operatorname{Epk} \pi}^{\mathcal{Z}} \cdot K_{m, \operatorname{Epk} \sigma}^{\mathcal{Z}} K_{n \in S(\pi, \sigma)}^{\mathcal{Z}}=\sum_{\Lambda \in \mathbf{L}_{n+m}} \underbrace{}_{=|\{\tau \in S(\pi, \sigma) \mid \operatorname{Epk} \tau=\Lambda\}| K_{n+m, \Lambda}^{\mathcal{Z}}} \underbrace{}_{\substack{\tau \in S(\pi, \sigma) ; \\
\operatorname{Epk} \tau=\Lambda}} K_{n+m, \Lambda}^{\mathcal{Z}} \\
& =\sum_{\Lambda \in \mathbf{L}_{n+m}}|\{\tau \in S(\pi, \sigma) \mid \operatorname{Epk} \tau=\Lambda\}| K_{n+m, \Lambda}^{\mathcal{Z}} .
\end{aligned}
$$


The same argument (but using $\pi^{\prime}$ and $\sigma^{\prime}$ instead of $\pi$ and $\sigma$ ) yields

$$
K_{n, \operatorname{Epk}\left(\pi^{\prime}\right)}^{\mathcal{Z}} \cdot K_{m, \operatorname{Epk}\left(\sigma^{\prime}\right)}^{\mathcal{Z}}=\sum_{\Lambda \in \mathbf{L}_{n+m}}\left|\left\{\tau \in S\left(\pi^{\prime}, \sigma^{\prime}\right) \mid \operatorname{Epk} \tau=\Lambda\right\}\right| K_{n+m, \Lambda}^{\mathcal{Z}}
$$

The left-hand sides of these two equalities are identical (since Epk $\pi=\operatorname{Epk}\left(\pi^{\prime}\right)$ and Epk $\left.\sigma=\operatorname{Epk}\left(\sigma^{\prime}\right)\right)$. Thus, their right-hand sides must also be identical. In other words, we have

$$
\begin{aligned}
& \sum_{\Lambda \in \mathbf{L}_{n+m}}|\{\tau \in S(\pi, \sigma) \mid \operatorname{Epk} \tau=\Lambda\}| K_{n+m, \Lambda}^{\mathcal{Z}} \\
= & \sum_{\Lambda \in \mathbf{L}_{n+m}}\left|\left\{\tau \in S\left(\pi^{\prime}, \sigma^{\prime}\right) \mid \operatorname{Epk} \tau=\Lambda\right\}\right| K_{n+m, \Lambda}^{\mathcal{Z}} .
\end{aligned}
$$

Since the family $\left(K_{n+m, \Lambda}^{\mathcal{Z}}\right)_{\Lambda \in \mathbf{L}_{n+m}}$ is $\mathbb{Q}$-linearly independent (by Proposition 56), this shows that

$$
|\{\tau \in S(\pi, \sigma) \mid \operatorname{Epk} \tau=\Lambda\}|=\left|\left\{\tau \in S\left(\pi^{\prime}, \sigma^{\prime}\right) \mid \operatorname{Epk} \tau=\Lambda\right\}\right|
$$

for each $\Lambda \in \mathbf{L}_{n+m}$. In other words, the multiset $\{\operatorname{Epk} \tau \mid \tau \in S(\pi, \sigma)\}_{\text {multi }}$ equals the multiset

$\left\{\text { Epk } \tau \mid \tau \in S\left(\pi^{\prime}, \sigma^{\prime}\right)\right\}_{\text {multi }}$ (because both of these multisets consist of elements of $\mathbf{L}_{n+m}$, and the previous sentence shows that each of these elements appears with equal multiplicities in them). This completes our proof of Theorem 60 .

We end this section with a tangential remark for readers of [4]:

Remark 61. Let us use the notations of [4] (specifically, the concept of "equivalent" statistics defined in [4, Section 3.1]; and various specific statistics defined in [4, Section 2.2]). The permutation statistics (Lpk, val), (Lpk, udr) and (Pk, udr) are equivalent to Epk, and therefore are shuffle-compatible.

Proof of Remark 61 (sketched). If $\mathrm{st}_{1}$ and $\mathrm{st}_{2}$ are two permutation statistics, then we shall write $\mathrm{st}_{1} \sim \mathrm{st}_{2}$ to mean "st. is equivalent to $\mathrm{st}_{2}$ ".

The permutation statistic val is equivalent to epk, because of [4, Lemma 2.1 (e)]. In other words, val $\sim$ epk. Hence, $(\mathrm{Lpk}, \mathrm{val}) \sim(\mathrm{Lpk}, \mathrm{epk})$. But if $\pi$ is an $n$-permutation, then Epk $\pi$ can be computed from the knowledge of Lpk $\pi$ and epk $\pi$ (indeed, Epk $\pi$ differs from Lpk $\pi$ only in the possible element $n$, so that

$$
\operatorname{Epk} \pi= \begin{cases}\operatorname{Lpk} \pi, & \text { if epk } \pi=|\operatorname{Lpk} \pi| ; \\ \operatorname{Lpk} \pi \cup\{n\}, & \text { if epk } \pi \neq|\operatorname{Lpk} \pi|\end{cases}
$$

) and vice versa $(\operatorname{since} \operatorname{Lpk} \pi=(\operatorname{Epk} \pi) \backslash\{n\}$ and epk $\pi=|\operatorname{Epk} \pi|)$. Thus, (Lpk, epk) Epk. Hence, altogether, we obtain $(\mathrm{Lpk}, \mathrm{val}) \sim(\mathrm{Lpk}, \mathrm{epk}) \sim$ Epk.

Moreover, [4, Lemma 2.2 (a)] shows that for any permutation $\pi$, the knowledge of $\operatorname{Lpk} \pi$ allows us to compute udr $\pi$ from val $\pi$ and vice versa. Hence, (Lpk, udr) $(\mathrm{Lpk}, \mathrm{val}) \sim$ Epk. 
On the other hand, $(\mathrm{Pk}, \mathrm{lpk}) \sim$ Lpk. (This is proven similarly to our proof of $($ Lpk, epk $) \sim$ Epk.)

Also, udr $\sim$ (lpk, val) (indeed, [4, Lemma 2.2 (b) and (c)] show how the value (lpk, val) $(\pi)$ can be computed from udr $\pi$, whereas [4, Lemma 2.2 (a)] shows the opposite direction $)$. Hence, $(\mathrm{Pk}, \mathrm{udr}) \sim(\mathrm{Pk}, \mathrm{lpk}, \mathrm{val}) \sim(\mathrm{Lpk}, \mathrm{val})($ since $(\mathrm{Pk}, \mathrm{lpk}) \sim \mathrm{Lpk})$. Therefore, $(\mathrm{Pk}, \mathrm{udr}) \sim(\mathrm{Lpk}, \mathrm{val}) \sim$ Epk.

We have now shown that the statistics (Lpk, val), (Lpk, udr) and (Pk, udr) are equivalent to Epk. Thus, [4, Theorem 3.2] shows that they are shuffle-compatible (since Epk is shuffle-compatible). This proves Remark 61.

Question 62. Our concept of a "ZZ-enriched $(P, \gamma)$-partition" generalizes the concept of an "enriched $(P, \gamma)$-partition" by restricting ourselves to a subset $\mathcal{Z}$ of $\mathcal{N} \times\{+,-\}$. (This does not sound like much of a generalization when stated like this, but as we have seen the behavior of the power series $\Gamma_{\mathcal{Z}}(P, \gamma)$ depends strongly on what $\mathcal{Z}$ is, and is not all anticipated by the $\mathcal{Z}=\mathcal{N} \times\{+,-\}$ case.) A different generalization of enriched $(P, \gamma)$ partitions (introduced by Hsiao and Petersen in [9]) are the colored $(P, \gamma)$-partitions, where the two-element set $\{+,-\}$ is replaced by the set $\left\{1, \omega, \ldots, \omega^{m-1}\right\}$ of all $m$-th roots of unity (where $m$ is a chosen positive integer, and $\omega$ is a fixed primitive $m$-th root of unity). We can play various games with this concept. The most natural thing to do seems to be to consider $m$ arbitrary total orders $<_{0},<_{1}, \ldots,<_{m-1}$ on the codomain $A$ of the labeling $\gamma$ (perhaps with some nice properties such as all intervals being finite) and an arbitrary subset $\mathcal{Z}$ of $\mathcal{N} \times\left\{1, \omega, \ldots, \omega^{m-1}\right\}$, and define a $\mathcal{Z}$-enriched colored $(P, \gamma)$-partition to be a map $f: P \rightarrow \mathcal{Z}$ such that every $x<y$ in $P$ satisfy the following conditions:

(i) We have $f(x) \preccurlyeq f(y)$. (Here, the total order on $\mathcal{N} \times\left\{1, \omega, \ldots, \omega^{m-1}\right\}$ is defined by

$$
\left(n, \omega^{i}\right) \prec\left(n^{\prime}, \omega^{i^{\prime}}\right) \text { if and only if either } n \prec n^{\prime} \text { or }\left(n=n^{\prime} \text { and } i<i^{\prime}\right)
$$

(for $\left.i, i^{\prime} \in\{0,1, \ldots, m-1\}\right)$.)

(ii) If $f(x)=f(y)=\left(n, \omega^{i}\right)$ for some $n \in \mathcal{N}$ and $i \in\{0,1, \ldots, m-1\}$, then $\gamma(x)<_{i}$ $\gamma(y)$.

Is this a useful concept, and can it be used to study permutation statistics?

Question 63. Corollary 54 provides a formula for rewriting a product of the form $K_{n, \Lambda}^{\mathcal{Z}}$. $K_{m, \Omega}^{\mathcal{Z}}$ as a $\mathbb{Q}$-linear combination of $K_{n+m, \Xi}^{\mathcal{Z}}$ 's when $\Lambda \in \mathbf{L}_{n}$ and $\Omega \in \mathbf{L}_{m}$ (because any such $\Lambda$ and $\Omega$ can be written as $\Lambda=\operatorname{Epk} \pi$ and $\Omega=\operatorname{Epk} \sigma$ for appropriate permutations $\pi$ and $\sigma)$. Thus, in particular, any such product belongs to the $\mathbb{Q}$-linear span of the $K_{n+m, \Xi}^{\mathcal{Z}}$ 's. Is this still true if $\Lambda$ and $\Omega$ are arbitrary subsets of $[n]$ and $[m]$ rather than having to belong to $\mathbf{L}_{n}$ and to $\mathbf{L}_{m}$ ? Computations with SageMath suggest that the answer is "yes". For example,

$$
\begin{aligned}
K_{2,\{1,2\}}^{\mathcal{Z}} \cdot K_{1,\{1\}}^{\mathcal{Z}} & =K_{3,\{2\}}^{\mathcal{Z}}+2 \cdot K_{3,\{1,3\}}^{\mathcal{Z}} \quad \text { and } \\
K_{2, \varnothing}^{\mathcal{Z}} \cdot K_{1,\{1\}}^{\mathcal{Z}} & =K_{3, \varnothing}^{\mathcal{Z}}+K_{3,\{2\}}^{\mathcal{Z}}+K_{3,\{1,3\}}^{\mathcal{Z}}=K_{3,\{1\}}^{\mathcal{Z}}+K_{3,\{2\}}^{\mathcal{Z}}+K_{3,\{3\}}^{\mathcal{Z}} .
\end{aligned}
$$

Note that the $\mathbb{Q}$-linear span of the $K_{n+m, \Xi}^{\mathcal{Z}}$ 's for all $\Xi \subseteq[n+m]$ is (generally) larger than that of the $K_{n+m, \Xi}^{\mathcal{Z}}$ 's with $\Xi \in \mathbf{L}_{n+m}$. 


\section{LR-shuffle-compatibility}

In this section, we shall introduce the concept of "LR-shuffle-compatibility" (short for "left-and-right-shuffle-compatibility"), which is stronger than usual shuffle-compatibility. We shall prove that Epk still is LR-shuffle-compatible, and study some other statistics that are and some that are not.

\subsection{Left and right shuffles}

We begin by introducing "left shuffles" and "right shuffles". There is a well-known notion of left and right shuffles of words (see, e.g., the operations $\prec$ and $\succ$ in [3, Example 1]). Specialized to permutations, it can be defined in the following simple way:

Definition 64. Let $\pi$ and $\sigma$ be two disjoint permutations. Then:

- A left shuffle of $\pi$ and $\sigma$ means a shuffle $\tau$ of $\pi$ and $\sigma$ such that the first letter of $\tau$ is the first letter of $\pi$. (This makes sense only when $\pi$ is nonempty. Otherwise, there are no left shuffles of $\pi$ and $\sigma$.)

- A right shuffle of $\pi$ and $\sigma$ means a shuffle $\tau$ of $\pi$ and $\sigma$ such that the first letter of $\tau$ is the first letter of $\sigma$. (This makes sense only when $\sigma$ is nonempty. Otherwise, there are no right shuffles of $\pi$ and $\sigma$.)

- We let $S_{\prec}(\pi, \sigma)$ denote the set of all left shuffles of $\pi$ and $\sigma$.

- We let $S_{\succ}(\pi, \sigma)$ denote the set of all right shuffles of $\pi$ and $\sigma$.

For example, the left shuffles of the two disjoint permutations $(3,1)$ and $(2,6)$ are

$$
(3,1,2,6), \quad(3,2,1,6), \quad(3,2,6,1),
$$

whereas their right shuffles are

$$
(2,3,1,6), \quad(2,3,6,1), \quad(2,6,3,1) .
$$

The permutations () and $(1,3)$ have only one right shuffle, which is $(1,3)$, and they have no left shuffles.

Clearly, if $\pi$ and $\sigma$ are two disjoint permutations such that at least one of $\pi$ and $\sigma$ is nonempty, then the two sets $S_{\prec}(\pi, \sigma)$ and $S_{\succ}(\pi, \sigma)$ are disjoint and their union is $S(\pi, \sigma)$ (because every shuffle of $\pi$ and $\sigma$ is either a left shuffle or a right shuffle, but not both).

Left and right shuffles have a recursive structure that makes them amenable to inductive arguments. To state it, we need one more definition:

Definition 65. Let $n \in \mathbb{N}$. Let $\pi$ be an $n$-permutation.

(a) For each $i \in\{1,2, \ldots, n\}$, we let $\pi_{i}$ denote the $i$-th entry of $\pi$. Thus, $\pi=$ $\left(\pi_{1}, \pi_{2}, \ldots, \pi_{n}\right)$. 
(b) If $a$ is a positive integer that does not appear in $\pi$, then $a: \pi$ denotes the $(n+1)$ permutation $\left(a, \pi_{1}, \pi_{2}, \ldots, \pi_{n}\right)$.

(c) If $n>0$, then $\pi_{\sim 1}$ denotes the $(n-1)$-permutation $\left(\pi_{2}, \pi_{3}, \ldots, \pi_{n}\right)$.

Proposition 66. Let $\pi$ and $\sigma$ be two disjoint permutations.

(a) We have $S_{\prec}(\pi, \sigma)=S_{\succ}(\sigma, \pi)$.

(b) If $\pi$ is nonempty, then the permutations $\pi_{\sim 1}$ and $\pi_{1}: \sigma$ are well-defined and disjoint, and satisfy $S_{\prec}(\pi, \sigma)=S_{\succ}\left(\pi_{\sim 1}, \pi_{1}: \sigma\right)$.

(c) If $\sigma$ is nonempty, then the permutations $\sigma_{\sim 1}$ and $\sigma_{1}: \pi$ are well-defined and disjoint, and satisfy $S_{\succ}(\pi, \sigma)=S_{\prec}\left(\sigma_{1}: \pi, \sigma_{\sim 1}\right)$.

Proof of Proposition 66. The fairly simple proof is left to the reader, who can also find it in [7].

\subsection{LR-shuffle-compatibility}

We shall use the so-called Iverson bracket notation for truth values:

Definition 67. If $\mathcal{A}$ is any logical statement, then we define an integer $[\mathcal{A}] \in\{0,1\}$ by

$$
[\mathcal{A}]=\left\{\begin{array}{ll}
1, & \text { if } \mathcal{A} \text { is true } \\
0, & \text { if } \mathcal{A} \text { is false }
\end{array} .\right.
$$

This integer $[\mathcal{A}]$ is known as the truth value of $\mathcal{A}$.

Thus, for example, $[4>2]=1$ whereas $[2>4]=0$.

We can now define a notion similar to shuffle-compatibility:

Definition 68. Let st be a permutation statistic. We say that st is LR-shuffle-compatible if and only if it has the following property: For any two disjoint nonempty permutations $\pi$ and $\sigma$, the multisets

$$
\left\{\text { st } \tau \mid \tau \in S_{\prec}(\pi, \sigma)\right\}_{\text {multi }} \quad \text { and } \quad\left\{\text { st } \tau \mid \tau \in S_{\succ}(\pi, \sigma)\right\}_{\text {multi }}
$$

depend only on st $\pi$, st $\sigma,|\pi|,|\sigma|$ and $\left[\pi_{1}>\sigma_{1}\right]$.

In other words, a permutation statistic st is LR-shuffle-compatible if and only if every two disjoint nonempty permutations $\pi$ and $\sigma$ and every two disjoint nonempty permutations $\pi^{\prime}$ and $\sigma^{\prime}$ satisfying

$$
\begin{aligned}
\text { st } \pi & =\operatorname{st}\left(\pi^{\prime}\right), & \text { st } \sigma=\operatorname{st}\left(\sigma^{\prime}\right), & \\
|\pi| & =\left|\pi^{\prime}\right|, & |\sigma|=\left|\sigma^{\prime}\right| \quad \text { and } & {\left[\pi_{1}>\sigma_{1}\right]=\left[\pi_{1}^{\prime}>\sigma_{1}^{\prime}\right] }
\end{aligned}
$$


satisfy

$$
\begin{array}{l|l|l}
\{\text { st } \tau & \left.\tau \in S_{\prec}(\pi, \sigma)\right\}_{\text {multi }}=\{\text { st } \tau \mid & \left.\tau \in S_{\prec}\left(\pi^{\prime}, \sigma^{\prime}\right)\right\}_{\text {multi }} \\
\{\text { st } \tau & \left.\tau \in S_{\succ}(\pi, \sigma)\right\}_{\text {multi }}=\{\text { st } \tau \mid & \left.\tau \in S_{\succ}\left(\pi^{\prime}, \sigma^{\prime}\right)\right\}_{\text {multi }} .
\end{array}
$$

For example, the permutation statistic $\mathrm{Pk}$ is not LR-shuffle-compatible. Indeed, if we take $\pi=(4,2,3), \sigma=(1), \pi^{\prime}=(2,3,4)$ and $\sigma^{\prime}=(1)$, then the equalities

$$
\begin{aligned}
& \operatorname{Pk} \pi=\operatorname{Pk}\left(\pi^{\prime}\right), \quad \operatorname{Pk} \sigma=\operatorname{Pk}\left(\sigma^{\prime}\right), \\
& |\pi|=\left|\pi^{\prime}\right|, \quad|\sigma|=\left|\sigma^{\prime}\right| \quad \text { and } \quad\left[\pi_{1}>\sigma_{1}\right]=\left[\pi_{1}^{\prime}>\sigma_{1}^{\prime}\right]
\end{aligned}
$$

are all satisfied, but

$$
\left\{\mathrm{Pk} \tau \mid \tau \in S_{\succ}(\pi, \sigma)\right\}_{\text {multi }}=\{\underbrace{\operatorname{Pk}(1,4,2,3)}_{=\{2\}}\}_{\text {multi }}=\{\{2\}\}_{\text {multi }}
$$

is not the same as

$$
\left\{\operatorname{Pk} \tau \mid \tau \in S_{\succ}\left(\pi^{\prime}, \sigma^{\prime}\right)\right\}_{\text {multi }}=\{\underbrace{\operatorname{Pk}(1,2,3,4)}_{=\varnothing}\}_{\text {multi }}=\{\varnothing\}_{\text {multi }} .
$$

Similarly, the permutation statistic Rpk is not LR-shuffle-compatible. As we will see in Theorem 75 further below, the three statistics Des, Lpk and Epk are LR-shufflecompatible.

\subsection{Head-graft-compatibility}

We shall now define another compatibility concept for a permutation statistic, which will later prove a useful stepping stone for checking the LR-shuffle-compatibility of this statistic.

Definition 69. Let st be a permutation statistic. We say that st is head-graft-compatible if and only if it has the following property: For any nonempty permutation $\pi$ and any letter $a$ that does not appear in $\pi$, the element st $(a: \pi)$ depends only on st $\pi,|\pi|$ and $\left[a>\pi_{1}\right]$.

In other words, a permutation statistic st is head-graft-compatible if and only if every nonempty permutation $\pi$, every letter $a$ that does not appear in $\pi$, every nonempty permutation $\pi^{\prime}$ and every letter $a^{\prime}$ that does not appear in $\pi^{\prime}$ satisfying

$$
\text { st } \pi=\operatorname{st}\left(\pi^{\prime}\right), \quad|\pi|=\left|\pi^{\prime}\right| \quad \text { and } \quad\left[a>\pi_{1}\right]=\left[a^{\prime}>\pi_{1}^{\prime}\right]
$$

satisfy st $(a: \pi)=\operatorname{st}\left(a^{\prime}: \pi^{\prime}\right)$. 
For example, the permutation statistic $\mathrm{Pk}$ is not head-graft-compatible, because if we take $\pi=(3,1), a=2, \pi^{\prime}=(3,4)$ and $a^{\prime}=2$, then we do have

$$
\operatorname{Pk} \pi=\operatorname{Pk}\left(\pi^{\prime}\right), \quad|\pi|=\left|\pi^{\prime}\right| \quad \text { and } \quad\left[a>\pi_{1}\right]=\left[a^{\prime}>\pi_{1}^{\prime}\right]
$$

but we don't have $\operatorname{Pk}(a: \pi)=\operatorname{Pk}\left(a^{\prime}: \pi^{\prime}\right)$ (in fact, $\operatorname{Pk}(a: \pi)=\operatorname{Pk}(2,3,1)=\{2\}$ whereas $\left.\operatorname{Pk}\left(a^{\prime}: \pi^{\prime}\right)=\operatorname{Pk}(2,3,4)=\varnothing\right)$. Similarly, it can be shown that Rpk is not head-graftcompatible. As we will see below (in Proposition 71), the permutation statistics Des, Lpk and Epk are head-graft-compatible; we will analyze a few other statistics in Subsection 3.5 .

Remark 70 . Let st be a head-graft-compatible permutation statistic. Then, it is easy to see that

$$
\text { st }(3,1,2)=\operatorname{st}(2,1,3) \quad \text { and } \quad \text { st }(2,3,1)=\operatorname{st}(1,3,2) \text {. }
$$

Moreover, these are the only restrictions that head-graft-compatibility places on the values of st at 3-permutations. The restrictions placed on the values of st at permutations of length $n>3$ are more complicated, and depend on its values on shorter permutations.

It is usually easy to check if a given permutation statistic is head-graft-compatible. For example:

\section{Proposition 71.}

(a) The permutation statistic Des is head-graft-compatible.

(b) The permutation statistic Lpk is head-graft-compatible.

(c) The permutation statistic Epk is head-graft-compatible.

Proof of Proposition 71. In this proof, we shall use the following notation: If $S$ is a set of integers, and $p$ is an integer, then $S+p$ shall denote the set $\{s+p \mid s \in S\}$.

(a) Let $\pi$ be a nonempty permutation. Let $a$ be a letter that does not appear in $\pi$. We shall express the element Des $(a: \pi)$ in terms of Des $\pi,|\pi|$ and $\left[a>\pi_{1}\right]$.

Let $n=|\pi|$. Thus, $\pi=\left(\pi_{1}, \pi_{2}, \ldots, \pi_{n}\right)$. Therefore, $a: \pi=\left(a, \pi_{1}, \pi_{2}, \ldots, \pi_{n}\right)$. Hence, the descents of $a: \pi$ are obtained as follows:

- The number 1 is a descent of $a: \pi$ if and only if $a>\pi_{1}$.

- Adding 1 to each descent of $\pi$ yields a descent of $a: \pi$. (That is, if $i$ is a descent of $\pi$, then $i+1$ is a descent of $a: \pi$.)

These are all the descents of $a: \pi$. Thus,

$$
\operatorname{Des}(a: \pi)=\left\{1 \mid a>\pi_{1}\right\} \cup(\operatorname{Des} \pi+1) .
$$

(The strange notation " $\left\{1 \mid a>\pi_{1}\right\}$ " means exactly what it says: It is the set of all numbers 1 satisfying $a>\pi_{1}$. In other words, it is $\{1\}$ if $a>\pi_{1}$, and $\varnothing$ otherwise.) 
The equality (12) shows that Des $(a: \pi)$ depends only on Des $\pi,|\pi|$ and $\left[a>\pi_{1}\right]$ (indeed, the truth value $\left[a>\pi_{1}\right]$ determines whether $a>\pi_{1}$ is true). In other words, Des is head-graft-compatible (by the definition of "head-graft-compatible"). This proves Proposition 71 (a).

(b) Let $\pi$ be a nonempty permutation. Let $a$ be a letter that does not appear in $\pi$. We shall express the element $\operatorname{Lpk}(a: \pi)$ in terms of $\operatorname{Lpk} \pi,|\pi|$ and $\left[a>\pi_{1}\right]$.

Notice first that $a \neq \pi_{1}$ (since $a$ does not appear in $\pi$ ). Thus, $a<\pi_{1}$ is true if and only if $a>\pi_{1}$ is false.

Let $n=|\pi|$. Therefore, $\pi=\left(\pi_{1}, \pi_{2}, \ldots, \pi_{n}\right)$. Thus, $a: \pi=\left(a, \pi_{1}, \pi_{2}, \ldots, \pi_{n}\right)$. Hence, the left peaks of $a: \pi$ are obtained as follows:

- The number 1 is a left peak of $a: \pi$ if and only if $a>\pi_{1}$.

- Adding 1 to each left peak $i$ of $\pi$ yields a left peak $i+1$ of $a: \pi$, except for the case when $i=1$ (in which case $i+1=2$ is a left peak of $a: \pi$ only if $a<\pi_{1}$ ).

These are all the left peaks of $a: \pi$. Thus,

$$
\operatorname{Lpk}(a: \pi)=\left\{1 \mid a>\pi_{1}\right\} \cup\left\{\begin{array}{ll}
\operatorname{Lpk} \pi+1, & \text { if } a<\pi_{1} ; \\
(\operatorname{Lpk} \pi+1) \backslash\{2\}, & \text { if not } a<\pi_{1}
\end{array} .\right.
$$

This equality shows that $\operatorname{Lpk}(a: \pi)$ depends only on $\operatorname{Lpk} \pi,|\pi|$ and $\left[a>\pi_{1}\right]$ (indeed, the truth value $\left[a>\pi_{1}\right]$ determines whether $a>\pi_{1}$ is true and also determines whether $a<\pi_{1}$ is $\operatorname{true}^{6}$ ). In other words, Lpk is head-graft-compatible (by the definition of "head-graft-compatible"). This proves Proposition 71 (b).

(c) To obtain a proof of Proposition 71 (c), it suffices to take our above proof of Proposition 71 (b) and replace every appearance of "left peak" and "Lpk" by "exterior peak" and "Epk".

\subsection{Proving LR-shuffle-compatibility}

Let us now state a sufficient criterion for the LR-shuffle-compatibility of a statistic:

Theorem 72. Let st be a permutation statistic that is both shuffle-compatible and headgraft-compatible. Then, st is LR-shuffle-compatible.

Before we prove this theorem, let us introduce some terminology and state an almosttrivial fact:

\section{Definition 73.}

(a) If $A$ is a finite multiset, and if $g$ is any object, then $|A|_{g}$ means the multiplicity of $g$ in $A$.

\footnotetext{
${ }^{6}$ Indeed, $a<\pi_{1}$ is true if and only if $a>\pi_{1}$ is false.
} 
(b) If $A$ and $B$ are two finite multisets, then we say that $B \subseteq A$ if and only if each object $g$ satisfies $|B|_{g} \leqslant|A|_{g}$.

(c) If $A$ and $B$ are two finite multisets satisfying $B \subseteq A$, then $A-B$ shall denote the "multiset difference" of $A$ and $B$; this is the finite multiset $C$ such that each object $g$ satisfies $|C|_{g}=|A|_{g}-|B|_{g}$. $\{1,2\}_{\text {multi }}$.

For example, $\{2,3,3\}_{\text {multi }} \subseteq\{1,2,2,3,3\}_{\text {multi }}$ and $\{1,2,2,3,3\}_{\text {multi }}-\{2,3,3\}_{\text {multi }}=$

Lemma 74. Let $\pi$ and $\sigma$ be two disjoint permutations such that at least one of $\pi$ and $\sigma$ is nonempty. Let st be any permutation statistic. Then:

(a) We have

$$
\begin{aligned}
& \left\{\text { st } \tau \mid \tau \in S_{\prec}(\pi, \sigma)\right\}_{\text {multi }} \\
& =\{\text { st } \tau \mid \tau \in S(\pi, \sigma)\}_{\text {multi }}-\left\{\text { st } \tau \mid \tau \in S_{\succ}(\pi, \sigma)\right\}_{\text {multi }} .
\end{aligned}
$$

(b) We have

$$
\begin{aligned}
& \left\{\text { st } \tau \mid \tau \in S_{\succ}(\pi, \sigma)\right\}_{\text {multi }} \\
& =\{\operatorname{st} \tau \mid \tau \in S(\pi, \sigma)\}_{\text {multi }}-\left\{\text { st } \tau \mid \tau \in S_{\prec}(\pi, \sigma)\right\}_{\text {multi }} .
\end{aligned}
$$

Proof of Lemma 74. Recall that the two sets $S_{\prec}(\pi, \sigma)$ and $S_{\succ}(\pi, \sigma)$ are disjoint and their union is $S(\pi, \sigma)$. Thus, $S_{\succ}(\pi, \sigma) \subseteq S(\pi, \sigma)$ and $S_{\prec}(\pi, \sigma)=S(\pi, \sigma) \backslash S_{\succ}(\pi, \sigma)$. Hence,

$$
\begin{aligned}
& \left\{\text { st } \tau \mid \tau \in S_{\prec}(\pi, \sigma)\right\}_{\text {multi }} \\
& =\{\text { st } \tau \mid \tau \in S(\pi, \sigma)\}_{\text {multi }}-\left\{\text { st } \tau \mid \tau \in S_{\succ}(\pi, \sigma)\right\}_{\text {multi }} .
\end{aligned}
$$

This proves Lemma 74 (a). The proof of Lemma 74 (b) is analogous.

Proof of Theorem 72. We shall first show the following:

Claim 1: Let $\pi, \pi^{\prime}$ and $\sigma$ be three nonempty permutations. Assume that $\pi$ and $\sigma$ are disjoint. Assume that $\pi^{\prime}$ and $\sigma$ are disjoint. Assume furthermore that

$$
\text { st } \pi=\operatorname{st}\left(\pi^{\prime}\right), \quad|\pi|=\left|\pi^{\prime}\right| \quad \text { and } \quad\left[\pi_{1}>\sigma_{1}\right]=\left[\pi_{1}^{\prime}>\sigma_{1}\right] .
$$

Then,

$$
\begin{aligned}
& \left\{\text { st } \tau \mid \tau \in S_{\prec}(\pi, \sigma)\right\}_{\text {multi }} \\
& =\left\{\text { st } \tau \mid \tau \in S_{\prec}\left(\pi^{\prime}, \sigma\right)\right\}_{\text {multi }}
\end{aligned}
$$

and

$$
\begin{aligned}
& \left\{\text { st } \tau \mid \tau \in S_{\succ}(\pi, \sigma)\right\}_{\text {multi }} \\
& =\left\{\operatorname{st} \tau \mid \tau \in S_{\succ}\left(\pi^{\prime}, \sigma\right)\right\}_{\text {multi }} .
\end{aligned}
$$


[Proof of Claim 1: We shall prove Claim 1 by induction on $|\sigma|$ :

Induction base: The case $|\sigma|=0$ cannot happen (because we have assumed $\sigma$ to be nonempty). Thus, Claim 1 is true in the case $|\sigma|=0$. This completes the induction base.

Induction step: Let $N$ be a positive integer. Assume (as the induction hypothesis) that Claim 1 holds when $|\sigma|=N-1$. We must now prove that Claim 1 holds when $|\sigma|=N$.

Indeed, let $\pi, \pi^{\prime}$ and $\sigma$ be as in Claim 1, and assume that $|\sigma|=N$. We must prove (14) and (15).

Proposition 66 (c) yields that the permutations $\sigma_{\sim 1}$ and $\sigma_{1}: \pi$ are well-defined and disjoint, and satisfy

$$
S_{\succ}(\pi, \sigma)=S_{\prec}\left(\sigma_{1}: \pi, \sigma_{\sim 1}\right) .
$$

Furthermore, $\left|\sigma_{\sim 1}\right|=|\sigma|-1=N-1$ (since $\left.|\sigma|=N\right)$.

Proposition 66 (c) (applied to $\pi^{\prime}$ instead of $\pi$ ) yields that the permutations $\sigma_{\sim 1}$ and $\sigma_{1}: \pi^{\prime}$ are well-defined and disjoint, and satisfy

$$
S_{\succ}\left(\pi^{\prime}, \sigma\right)=S_{\prec}\left(\sigma_{1}: \pi^{\prime}, \sigma_{\sim 1}\right) .
$$

The letter $\sigma_{1}$ does not appear in the permutation $\pi$ (since $\pi$ and $\sigma$ are disjoint). Similarly, the letter $\sigma_{1}$ does not appear in the permutation $\pi^{\prime}$. Also, $\left|\sigma_{1}: \pi\right|=\underbrace{|\pi|}_{=\left|\pi^{\prime}\right|}+1=$ $\left|\pi^{\prime}\right|+1=\left|\sigma_{1}: \pi^{\prime}\right|$

We have $\sigma_{1} \neq \pi_{1}$ (since $\pi$ and $\sigma$ are disjoint). Thus, the statement $\left(\sigma_{1}>\pi_{1}\right)$ is equivalent to (not $\pi_{1}>\sigma_{1}$ ). Hence, $\left[\sigma_{1}>\pi_{1}\right]=\left[\right.$ not $\left.\pi_{1}>\sigma_{1}\right]=1-\left[\pi_{1}>\sigma_{1}\right]$. Similarly, $\left[\sigma_{1}>\pi_{1}^{\prime}\right]=1-\left[\pi_{1}^{\prime}>\sigma_{1}\right]$. Hence,

$$
\left[\sigma_{1}>\pi_{1}\right]=1-\underbrace{\left[\pi_{1}>\sigma_{1}\right]}_{=\left[\pi_{1}^{\prime}>\sigma_{1}\right]}=1-\left[\pi_{1}^{\prime}>\sigma_{1}\right]=\left[\sigma_{1}>\pi_{1}^{\prime}\right] .
$$

Both permutations $\sigma_{1}: \pi$ and $\sigma_{1}: \pi^{\prime}$ begin with the letter $\sigma_{1}$. Thus, both $\left(\sigma_{1}: \pi\right)_{1}$ and $\left(\sigma_{1}: \pi^{\prime}\right)_{1}$ equal $\sigma_{1}$. Hence, $\left(\sigma_{1}: \pi\right)_{1}=\left(\sigma_{1}: \pi^{\prime}\right)_{1}$.

The statistic st is head-graft-compatible. In other words, for any nonempty permutation $\varphi$ and any letter $a$ that does not appear in $\varphi$, the element st $(a: \varphi)$ depends only on st $(\varphi),|\varphi|$ and $\left[a>\varphi_{1}\right]$ (by the definition of "head-graft-compatible"). Hence, if $\varphi$ and $\varphi^{\prime}$ are two nonempty permutations, and if $a$ is any letter that does not appear in $\varphi$ and does not appear in $\varphi^{\prime}$, and if we have st $\varphi=\operatorname{st}\left(\varphi^{\prime}\right)$ and $|\varphi|=\left|\varphi^{\prime}\right|$ and $\left[a>\varphi_{1}\right]=\left[a>\varphi_{1}^{\prime}\right]$, then st $(a: \varphi)=$ st $\left(a: \varphi^{\prime}\right)$. Applying this to $a=\sigma_{1}, \varphi=\pi$ and $\varphi^{\prime}=\pi^{\prime}$, we obtain

$$
\operatorname{st}\left(\sigma_{1}: \pi\right)=\operatorname{st}\left(\sigma_{1}: \pi^{\prime}\right)
$$

(since st $\pi=\operatorname{st}\left(\pi^{\prime}\right)$ and $|\pi|=\left|\pi^{\prime}\right|$ and $\left[\sigma_{1}>\pi_{1}\right]=\left[\sigma_{1}>\pi_{1}^{\prime}\right]$ ).

Next, we claim that

$$
\begin{aligned}
& \left\{\text { st } \tau \mid \tau \in S_{\prec}\left(\sigma_{1}: \pi, \sigma_{\sim 1}\right)\right\}_{\text {multi }} \\
& =\left\{\operatorname{st} \tau \mid \tau \in S_{\prec}\left(\sigma_{1}: \pi^{\prime}, \sigma_{\sim 1}\right)\right\}_{\text {multi }}
\end{aligned}
$$


[Proof of (18): The permutations $\sigma_{1}: \pi$ and $\sigma_{1}: \pi^{\prime}$ are clearly nonempty. Hence, if $\sigma_{\sim 1}$ is the 0-permutation (), then $S_{\prec}\left(\sigma_{1}: \pi, \sigma_{\sim 1}\right)=\left\{\sigma_{1}: \pi\right\}$ and $S_{\prec}\left(\sigma_{1}: \pi^{\prime}, \sigma_{\sim 1}\right)=\left\{\sigma_{1}: \pi^{\prime}\right\}$. Thus, if $\sigma_{\sim 1}$ is the 0-permutation (), then (18) follows from

$$
\begin{aligned}
& \left.\{\operatorname{st} \tau \mid \tau \in \underbrace{S_{\prec}\left(\sigma_{1}: \pi, \sigma_{\sim 1}\right)}_{=\left\{\sigma_{1}: \pi\right\}}\}\right\}_{\text {multi }} \underbrace{\operatorname{st}\left(\sigma_{1}: \pi\right)}_{=\operatorname{st}\left(\sigma_{1}: \pi^{\prime}\right)}\}_{\text {multi }} \\
& =\left\{\operatorname{st} \tau \mid \tau \in\left\{\sigma_{1}: \pi\right\}\right\}_{\text {multi }}=\{\underbrace{\left\{\sigma_{1}: \pi^{\prime}\right\}}_{=S_{\prec}\left(\sigma_{1}: \pi^{\prime}, \sigma_{\sim 1}\right)}\}_{\text {multi }} \\
& =\left\{\operatorname{st}\left(\sigma_{1}: \pi^{\prime}\right)\right\}_{\text {multi }}=\{\tau \in \\
& =\left\{\operatorname{st} \tau \mid \tau \in S_{\prec}\left(\sigma_{1}: \pi^{\prime}, \sigma_{\sim 1}\right)\right\}_{\text {multi }} \cdot
\end{aligned}
$$

Thus, for the rest of our proof of (18), we WLOG assume that $\sigma_{\sim 1}$ is not the 0 -permutation (). Thus, $\sigma_{\sim 1}$ is nonempty.

But recall that $\left|\sigma_{\sim 1}\right|=N-1$. Hence, the induction hypothesis allows us to apply Claim 1 to $\sigma_{1}$ : $\pi, \sigma_{1}: \pi^{\prime}$ and $\sigma_{\sim 1}$ instead of $\pi, \pi^{\prime}$ and $\sigma$ (because we know that the permutations $\sigma_{\sim 1}$ and $\sigma_{1}: \pi$ are disjoint; that the permutations $\sigma_{\sim 1}$ and $\sigma_{1}: \pi^{\prime}$ are disjoint; that $\operatorname{st}\left(\sigma_{1}: \pi\right)=\operatorname{st}\left(\sigma_{1}: \pi^{\prime}\right)$ and $\left|\sigma_{1}: \pi\right|=\left|\sigma_{1}: \pi^{\prime}\right| ;$ and that

$$
\begin{aligned}
& \left.[\underbrace{\left(\sigma_{1}: \pi\right)_{1}}_{=\left(\sigma_{1}: \pi^{\prime}\right)_{1}}>\left(\sigma_{\sim 1}\right)_{1}]=\left[\left(\sigma_{1}: \pi^{\prime}\right)_{1}>\left(\sigma_{\sim 1}\right)_{1}\right]\right) \text {. We therefore obtain } \\
& \left\{\text { st } \tau \mid \tau \in S_{\prec}\left(\sigma_{1}: \pi, \sigma_{\sim 1}\right)\right\}_{\text {multi }}=\left\{\operatorname{st} \tau \mid \tau \in S_{\prec}\left(\sigma_{1}: \pi^{\prime}, \sigma_{\sim 1}\right)\right\}_{\text {multi }}
\end{aligned}
$$

and

$$
\left\{\text { st } \tau \mid \tau \in S_{\succ}\left(\sigma_{1}: \pi, \sigma_{\sim 1}\right)\right\}_{\text {multi }}=\left\{\text { st } \tau \mid \tau \in S_{\succ}\left(\sigma_{1}: \pi^{\prime}, \sigma_{\sim 1}\right)\right\}_{\text {multi }} .
$$

The first of these two equalities is precisely (18). Thus, (18) is proven.]

Now,

$$
\begin{aligned}
& \{\text { st } \tau \mid \tau \in \underbrace{S_{\succ}(\pi, \sigma)}_{\substack{\left.S_{\prec}\left(\sigma_{1}: \pi, \sigma_{\sim 1}\right) \\
\text { (by }(16)\right)}}\} \\
& =\left\{\operatorname{st} \tau \mid \tau \in S_{\prec}\left(\sigma_{1}: \pi, \sigma_{\sim 1}\right)\right\}_{\text {multi }} \\
& =\{\text { st } \tau \mid \tau \in \underbrace{S_{\prec}\left(\sigma_{1}: \pi^{\prime}, \sigma_{\sim 1}\right)}_{\substack{\left.=S_{\succ}\left(\pi^{\prime}, \sigma\right) \\
\text { (by }(17)\right)}}\}
\end{aligned}
$$




$$
=\left\{\operatorname{st} \tau \mid \tau \in S_{\succ}\left(\pi^{\prime}, \sigma\right)\right\}_{\text {multi }} .
$$

This proves (15). It remains to prove (14).

Lemma 74 (a) yields

$$
\begin{aligned}
& \left\{\text { st } \tau \mid \tau \in S_{\prec}(\pi, \sigma)\right\}_{\text {multi }} \\
& =\{\text { st } \tau \mid \tau \in S(\pi, \sigma)\}_{\text {multi }}-\left\{\text { st } \tau \mid \tau \in S_{\succ}(\pi, \sigma)\right\}_{\text {multi }} .
\end{aligned}
$$

Lemma 74 (a) (applied to $\pi^{\prime}$ instead of $\pi$ ) yields

$$
\begin{aligned}
& \left\{\text { st } \tau \mid \tau \in S_{\prec}\left(\pi^{\prime}, \sigma\right)\right\}_{\text {multi }} \\
& =\left\{\operatorname{st} \tau \mid \tau \in S\left(\pi^{\prime}, \sigma\right)\right\}_{\text {multi }}-\left\{\text { st } \tau \mid \tau \in S_{\succ}\left(\pi^{\prime}, \sigma\right)\right\}_{\text {multi }} .
\end{aligned}
$$

But recall that the statistic st is shuffle-compatible. In other words, for any two disjoint permutations $\alpha$ and $\beta$, the multiset

$$
\{\text { st } \tau \mid \tau \in S(\alpha, \beta)\}_{\text {multi }}
$$

depends only on st $\alpha$, st $\beta,|\alpha|$ and $|\beta|$ (by the definition of shuffle-compatibility). In other words, if $\alpha$ and $\beta$ are two disjoint permutations, and if $\alpha^{\prime}$ and $\beta^{\prime}$ are two disjoint permutations, and if we have

$$
\operatorname{st} \alpha=\operatorname{st}\left(\alpha^{\prime}\right), \quad \quad \operatorname{st} \beta=\operatorname{st}\left(\beta^{\prime}\right), \quad|\alpha|=\left|\alpha^{\prime}\right| \quad \text { and } \quad|\beta|=\left|\beta^{\prime}\right|,
$$

then

$$
\{\text { st } \tau \mid \tau \in S(\alpha, \beta)\}_{\text {multi }}=\left\{\text { st } \tau \mid \tau \in S\left(\alpha^{\prime}, \beta^{\prime}\right)\right\}_{\text {multi }} .
$$

Applying this to $\alpha=\pi, \beta=\sigma, \alpha^{\prime}=\pi^{\prime}$ and $\beta^{\prime}=\sigma$, we obtain

$$
\{\text { st } \tau \mid \tau \in S(\pi, \sigma)\}_{\text {multi }}=\left\{\text { st } \tau \mid \tau \in S\left(\pi^{\prime}, \sigma\right)\right\}_{\text {multi }}
$$

(since st $\pi=\operatorname{st}\left(\pi^{\prime}\right)$, st $\sigma=\operatorname{st} \sigma,|\pi|=\left|\pi^{\prime}\right|$ and $|\sigma|=|\sigma|$ ). Now, (20) becomes

$$
\begin{aligned}
& \left\{\text { st } \tau \mid \tau \in S_{\prec}(\pi, \sigma)\right\}_{\text {multi }} \\
& =\underbrace{\{\operatorname{st} \tau \mid \tau \in S(\pi, \sigma)\}_{\text {multi }}}_{=\left\{\operatorname{st} \tau \mid \begin{array}{c}
\left.\mid \tau \in S\left(\pi^{\prime}, \sigma\right)\right\}_{\text {multi }} \\
(\text { by }(22))
\end{array}\right.}-\underbrace{\left\{\text { st } \tau \mid \tau \in S_{\succ}(\pi, \sigma)\right\}_{\text {multi }}}_{=\left\{\operatorname{st} \tau \mid \begin{array}{c}
\left.\tau \in S_{\succ}\left(\pi^{\prime}, \sigma\right)\right\}_{\text {multi }} \\
(\text { by }(19))
\end{array}\right.} \\
& =\left\{\operatorname{st} \tau \mid \tau \in S\left(\pi^{\prime}, \sigma\right)\right\}_{\text {multi }}-\left\{\operatorname{st} \tau \mid \tau \in S_{\succ}\left(\pi^{\prime}, \sigma\right)\right\}_{\text {multi }} \\
& \left.=\left\{\operatorname{st} \tau \mid \tau \in S_{\prec}\left(\pi^{\prime}, \sigma\right)\right\}_{\text {multi }} \quad \text { (by }(21)\right) \text {. }
\end{aligned}
$$

Thus, (14) is proven. Hence, we have proven both (14) and (15). This shows that Claim 1 holds for our $\pi, \pi^{\prime}$ and $\sigma$. This completes the induction step. Thus, Claim 1 is proven by induction.]

We shall next derive a "mirror version" of Claim 1: 
Claim 2: Let $\pi, \sigma$ and $\sigma^{\prime}$ be three nonempty permutations. Assume that $\pi$ and $\sigma$ are disjoint. Assume that $\pi$ and $\sigma^{\prime}$ are disjoint. Assume furthermore that

$$
\operatorname{st} \sigma=\operatorname{st}\left(\sigma^{\prime}\right), \quad|\sigma|=\left|\sigma^{\prime}\right| \quad \text { and } \quad\left[\pi_{1}>\sigma_{1}\right]=\left[\pi_{1}>\sigma_{1}^{\prime}\right] .
$$

Then,

$$
\begin{aligned}
& \left\{\text { st } \tau \mid \tau \in S_{\prec}(\pi, \sigma)\right\}_{\text {multi }} \\
& =\left\{\text { st } \tau \mid \tau \in S_{\prec}\left(\pi, \sigma^{\prime}\right)\right\}_{\text {multi }}
\end{aligned}
$$

and

$$
\begin{aligned}
& \left\{\text { st } \tau \mid \tau \in S_{\succ}(\pi, \sigma)\right\}_{\text {multi }} \\
& =\left\{\text { st } \tau \mid \tau \in S_{\succ}\left(\pi, \sigma^{\prime}\right)\right\}_{\text {multi }} .
\end{aligned}
$$

[Proof of Claim 2: We have $\sigma_{1} \neq \pi_{1}$ (since $\pi$ and $\sigma$ are disjoint). Thus, the statement $\left(\sigma_{1}>\pi_{1}\right)$ is equivalent to (not $\left.\pi_{1}>\sigma_{1}\right)$. Hence, $\left[\sigma_{1}>\pi_{1}\right]=\left[\operatorname{not} \pi_{1}>\sigma_{1}\right]=1-\left[\pi_{1}>\sigma_{1}\right]$. Similarly, $\left[\sigma_{1}^{\prime}>\pi_{1}\right]=1-\left[\pi_{1}>\sigma_{1}^{\prime}\right]$. Hence,

$$
\left[\sigma_{1}>\pi_{1}\right]=1-\underbrace{\left[\pi_{1}>\sigma_{1}\right]}_{=\left[\pi_{1}>\sigma_{1}^{\prime}\right]}=1-\left[\pi_{1}>\sigma_{1}^{\prime}\right]=\left[\sigma_{1}^{\prime}>\pi_{1}\right] .
$$

Hence, Claim 1 (applied to $\sigma, \sigma^{\prime}$ and $\pi$ instead of $\pi, \pi^{\prime}$ and $\sigma$ ) shows that

$$
\begin{aligned}
& \left\{\text { st } \tau \mid \tau \in S_{\prec}(\sigma, \pi)\right\}_{\text {multi }} \\
& =\left\{\text { st } \tau \mid \tau \in S_{\prec}\left(\sigma^{\prime}, \pi\right)\right\}_{\text {multi }}
\end{aligned}
$$

and

$$
\begin{aligned}
& \left\{\text { st } \tau \mid \tau \in S_{\succ}(\sigma, \pi)\right\}_{\text {multi }} \\
& =\left\{\text { st } \tau \mid \tau \in S_{\succ}\left(\sigma^{\prime}, \pi\right)\right\}_{\text {multi }} .
\end{aligned}
$$

But Proposition 66 (a) yields $S_{\prec}(\pi, \sigma)=S_{\succ}(\sigma, \pi)$. Similarly, $S_{\prec}\left(\pi, \sigma^{\prime}\right)=S_{\succ}\left(\sigma^{\prime}, \pi\right)$. Also, Proposition 66 (a) (applied to $\sigma$ and $\pi$ instead of $\pi$ and $\sigma$ ) yields $S_{\prec}(\sigma, \pi)=$ $S_{\succ}(\pi, \sigma)$. Similarly, $S_{\prec}\left(\sigma^{\prime}, \pi\right)=S_{\succ}\left(\pi, \sigma^{\prime}\right)$. Using all these equalities, we find

$$
\begin{aligned}
& \{\text { st } \tau \mid \tau \in \underbrace{S_{\prec}(\pi, \sigma)}_{=S_{\succ}(\sigma, \pi)}\}_{\text {multi }} \\
& =\left\{\operatorname{st} \tau \mid \tau \in S_{\succ}(\sigma, \pi)\right\}_{\text {multi }}=\{\operatorname{st} \tau \mid \tau \in \underbrace{S_{\succ}\left(\sigma^{\prime}, \pi\right)}_{=S_{\prec}\left(\pi, \sigma^{\prime}\right)}\}_{\text {multi }}
\end{aligned}
$$




$$
=\left\{\operatorname{st} \tau \mid \tau \in S_{\prec}\left(\pi, \sigma^{\prime}\right)\right\}_{\text {multi }}
$$

and

$$
\begin{aligned}
& \{\text { st } \tau \mid \tau \in \underbrace{S_{\succ}(\pi, \sigma)}_{=S_{\prec}(\sigma, \pi)}\}_{\text {multi }} \\
& =\left\{\operatorname{st} \tau \mid \tau \in S_{\prec}(\sigma, \pi)\right\}_{\text {multi }}=\{\operatorname{st} \tau \mid \tau \in \underbrace{S_{\prec}\left(\sigma^{\prime}, \pi\right)}_{=S_{\succ}\left(\pi, \sigma^{\prime}\right)}\}_{\text {multi }} \\
& =\left\{\operatorname{st} \tau \mid \tau \in S_{\succ}\left(\pi, \sigma^{\prime}\right)\right\}_{\text {multi }} .
\end{aligned}
$$

Thus, Claim 2 is proven.]

Finally, we are ready to take on the LR-shuffle-compatibility of st:

Claim 3: Let $\pi$ and $\sigma$ be two disjoint nonempty permutations. Let $\pi^{\prime}$ and $\sigma^{\prime}$ be two disjoint nonempty permutations. Assume that

$$
\begin{array}{rlrl}
\text { st } \pi & =\operatorname{st}\left(\pi^{\prime}\right), & \text { st } \sigma=\operatorname{st}\left(\sigma^{\prime}\right), & \\
|\pi| & =\left|\pi^{\prime}\right|, & |\sigma|=\left|\sigma^{\prime}\right| \quad \text { and } \quad\left[\pi_{1}>\sigma_{1}\right]=\left[\pi_{1}^{\prime}>\sigma_{1}^{\prime}\right] .
\end{array}
$$

Then,

$$
\left\{\text { st } \tau \mid \tau \in S_{\prec}(\pi, \sigma)\right\}_{\text {multi }}=\left\{\text { st } \tau \mid \tau \in S_{\prec}\left(\pi^{\prime}, \sigma^{\prime}\right)\right\}_{\text {multi }}
$$

and

$$
\left\{\text { st } \tau \mid \tau \in S_{\succ}(\pi, \sigma)\right\}_{\text {multi }}=\left\{\text { st } \tau \mid \tau \in S_{\succ}\left(\pi^{\prime}, \sigma^{\prime}\right)\right\}_{\text {multi }} .
$$

[Proof of Claim 3: We have $\left[\pi_{1}>\sigma_{1}\right]=\left[\pi_{1}^{\prime}>\sigma_{1}^{\prime}\right]$. Since $\left[\pi_{1}>\sigma_{1}\right]=\left[\pi_{1}^{\prime}>\sigma_{1}^{\prime}\right]$ is either 1 or 0 , we must therefore be in one of the following two cases:

Case 1: We have $\left[\pi_{1}>\sigma_{1}\right]=\left[\pi_{1}^{\prime}>\sigma_{1}^{\prime}\right]=1$.

Case 2: We have $\left[\pi_{1}>\sigma_{1}\right]=\left[\pi_{1}^{\prime}>\sigma_{1}^{\prime}\right]=0$.

Let us first consider Case 1. In this case, we have $\left[\pi_{1}>\sigma_{1}\right]=\left[\pi_{1}^{\prime}>\sigma_{1}^{\prime}\right]=1$.

There clearly exists a positive integer $N$ that is larger than all entries of $\sigma$ and larger than all entries of $\sigma^{\prime}$. Consider such an $N$. Let $n=|\pi|$; thus, $\pi=\left(\pi_{1}, \pi_{2}, \ldots, \pi_{n}\right)$. Let $\gamma$ be the permutation $\left(\pi_{1}+N, \pi_{2}+N, \ldots, \pi_{n}+N\right)$. This permutation $\gamma$ is order-isomorphic to $\pi$, but is disjoint from $\sigma$ (since all its entries are $>N$, while all the entries of $\sigma$ are $<N$ ) and disjoint from $\sigma^{\prime}$ (for similar reasons). Also, $\gamma_{1}=\underbrace{\pi_{1}}_{>0}+N>N>\sigma_{1}$ (since $N$ is larger than all entries of $\sigma$ ), so that $\left[\gamma_{1}>\sigma_{1}\right]=1$. Similarly, $\left[\gamma_{1}>\sigma_{1}^{\prime}\right]=1$.

The permutation $\gamma$ is order-isomorphic to $\pi$. Thus, st $\gamma=$ st $\pi$ (since st is a permutation statistic) and $|\gamma|=|\pi|$. The permutation $\gamma$ is furthermore nonempty (since it is order-isomorphic to the nonempty permutation $\pi)$. Also, st $\gamma=\operatorname{st} \pi=\operatorname{st}\left(\pi^{\prime}\right)$ and $|\gamma|=|\pi|=\left|\pi^{\prime}\right|$. Moreover, $\left[\pi_{1}>\sigma_{1}\right]=1=\left[\gamma_{1}>\sigma_{1}\right]$ and $\left[\gamma_{1}>\sigma_{1}\right]=1=\left[\gamma_{1}>\sigma_{1}^{\prime}\right]$ and $\left[\gamma_{1}>\sigma_{1}^{\prime}\right]=1=\left[\pi_{1}^{\prime}>\sigma_{1}^{\prime}\right]$. Hence, Claim 1 (applied to $\gamma$ instead of $\pi^{\prime}$ ) yields

$$
\left\{\text { st } \tau \mid \tau \in S_{\prec}(\pi, \sigma)\right\}_{\text {multi }}=\left\{\text { st } \tau \mid \tau \in S_{\prec}(\gamma, \sigma)\right\}_{\text {multi }}
$$


and

$$
\left\{\text { st } \tau \mid \tau \in S_{\succ}(\pi, \sigma)\right\}_{\text {multi }}=\left\{\operatorname{st} \tau \mid \tau \in S_{\succ}(\gamma, \sigma)\right\}_{\text {multi }} .
$$

Furthermore, Claim 2 (applied to $\gamma$ instead of $\pi$ ) yields

$$
\left\{\text { st } \tau \mid \tau \in S_{\prec}(\gamma, \sigma)\right\}_{\text {multi }}=\left\{\text { st } \tau \mid \tau \in S_{\prec}\left(\gamma, \sigma^{\prime}\right)\right\}_{\text {multi }}
$$

and

$$
\left\{\text { st } \tau \mid \tau \in S_{\succ}(\gamma, \sigma)\right\}_{\text {multi }}=\left\{\operatorname{st} \tau \mid \tau \in S_{\succ}\left(\gamma, \sigma^{\prime}\right)\right\}_{\text {multi }} .
$$

Finally, Claim 1 (applied to $\gamma$ and $\sigma^{\prime}$ instead of $\pi$ and $\sigma$ ) yields

$$
\left\{\text { st } \tau \mid \tau \in S_{\prec}\left(\gamma, \sigma^{\prime}\right)\right\}_{\text {multi }}=\left\{\text { st } \tau \mid \tau \in S_{\prec}\left(\pi^{\prime}, \sigma^{\prime}\right)\right\}_{\text {multi }}
$$

and

$$
\left\{\text { st } \tau \mid \tau \in S_{\succ}\left(\gamma, \sigma^{\prime}\right)\right\}_{\text {multi }}=\left\{\operatorname{st} \tau \mid \tau \in S_{\succ}\left(\pi^{\prime}, \sigma^{\prime}\right)\right\}_{\text {multi }} .
$$

Combining the equalities we have found, we obtain

$$
\begin{aligned}
\left\{\operatorname{st} \tau \mid \tau \in S_{\prec}(\pi, \sigma)\right\}_{\text {multi }} & =\left\{\text { st } \tau \mid \tau \in S_{\prec}(\gamma, \sigma)\right\}_{\text {multi }} \\
& =\left\{\operatorname{st} \tau \mid \tau \in S_{\prec}\left(\gamma, \sigma^{\prime}\right)\right\}_{\text {multi }} \\
& =\left\{\operatorname{st} \tau \mid \tau \in S_{\prec}\left(\pi^{\prime}, \sigma^{\prime}\right)\right\}_{\text {multi }}
\end{aligned}
$$

The same argument (but with the symbols " $S_{\prec}$ " and " $S_{\succ}$ " interchanged) yields

$$
\left\{\text { st } \tau \mid \tau \in S_{\succ}(\pi, \sigma)\right\}_{\text {multi }}=\left\{\text { st } \tau \mid \tau \in S_{\succ}\left(\pi^{\prime}, \sigma^{\prime}\right)\right\}_{\text {multi }} .
$$

Thus, Claim 3 is proven in Case 1.

Let us now consider Case 2. In this case, we have $\left[\pi_{1}>\sigma_{1}\right]=\left[\pi_{1}^{\prime}>\sigma_{1}^{\prime}\right]=0$.

There clearly exists a positive integer $N$ that is larger than all entries of $\pi$ and larger than all entries of $\pi^{\prime}$. Consider such an $N$. Set $m=|\sigma|$. Thus, $\sigma=\left(\sigma_{1}, \sigma_{2}, \ldots, \sigma_{m}\right)$. Let $\delta$ be the permutation $\left(\sigma_{1}+N, \sigma_{2}+N, \ldots, \sigma_{m}+N\right)$. This permutation $\delta$ is orderisomorphic to $\sigma$, but is disjoint from $\pi$ (since all its entries are $>N$, while all the entries of $\pi$ are $<N$ ) and disjoint from $\pi^{\prime}$ (for similar reasons). Also, $\delta_{1}=\underbrace{\sigma_{1}}_{>0}+N>N>\pi_{1}$ (since $N$ is larger than all entries of $\pi$ ), so that we don't have $\pi_{1}>\delta_{1}$. Thus, $\left[\pi_{1}>\delta_{1}\right]=0$. Similarly, $\left[\pi_{1}^{\prime}>\delta_{1}\right]=0$.

The permutation $\delta$ is order-isomorphic to $\sigma$. Thus, st $\delta=\operatorname{st} \sigma$ (since st is a permutation statistic) and $|\delta|=|\sigma|$. The permutation $\delta$ is furthermore nonempty (since it is orderisomorphic to the nonempty permutation $\sigma)$. Also, st $\delta=\operatorname{st} \sigma=\operatorname{st}\left(\sigma^{\prime}\right)$ and $|\delta|=|\sigma|=$ $\left|\sigma^{\prime}\right|$. Moreover, $\left[\pi_{1}>\sigma_{1}\right]=0=\left[\pi_{1}>\delta_{1}\right]$ and $\left[\pi_{1}>\delta_{1}\right]=0=\left[\pi_{1}^{\prime}>\delta_{1}\right]$ and $\left[\pi_{1}^{\prime}>\delta_{1}\right]=$ $0=\left[\pi_{1}^{\prime}>\sigma_{1}^{\prime}\right]$. Hence, Claim 2 (applied to $\delta$ instead of $\sigma^{\prime}$ ) yields

$$
\left\{\text { st } \tau \mid \tau \in S_{\prec}(\pi, \sigma)\right\}_{\text {multi }}=\left\{\text { st } \tau \mid \tau \in S_{\prec}(\pi, \delta)\right\}_{\text {multi }}
$$

and

$$
\left\{\text { st } \tau \mid \tau \in S_{\succ}(\pi, \sigma)\right\}_{\text {multi }}=\left\{\operatorname{st} \tau \mid \tau \in S_{\succ}(\pi, \delta)\right\}_{\text {multi }} .
$$


Furthermore, Claim 1 (applied to $\delta$ instead of $\sigma$ ) yields

$$
\left\{\text { st } \tau \mid \tau \in S_{\prec}(\pi, \delta)\right\}_{\text {multi }}=\left\{\text { st } \tau \mid \tau \in S_{\prec}\left(\pi^{\prime}, \delta\right)\right\}_{\text {multi }}
$$

and

$$
\left\{\text { st } \tau \mid \tau \in S_{\succ}(\pi, \delta)\right\}_{\text {multi }}=\left\{\text { st } \tau \mid \tau \in S_{\succ}\left(\pi^{\prime}, \delta\right)\right\}_{\text {multi }} .
$$

Finally, Claim 2 (applied to $\pi^{\prime}$ and $\delta$ instead of $\pi$ and $\sigma$ ) yields

$$
\left\{\text { st } \tau \mid \tau \in S_{\prec}\left(\pi^{\prime}, \delta\right)\right\}_{\text {multi }}=\left\{\text { st } \tau \mid \tau \in S_{\prec}\left(\pi^{\prime}, \sigma^{\prime}\right)\right\}_{\text {multi }}
$$

and

$$
\left\{\text { st } \tau \mid \tau \in S_{\succ}\left(\pi^{\prime}, \delta\right)\right\}_{\text {multi }}=\left\{\operatorname{st} \tau \mid \tau \in S_{\succ}\left(\pi^{\prime}, \sigma^{\prime}\right)\right\}_{\text {multi }} .
$$

Combining the equalities we have found, we obtain

$$
\begin{aligned}
\left\{\text { st } \tau \mid \tau \in S_{\prec}(\pi, \sigma)\right\}_{\text {multi }} & =\left\{\text { st } \tau \mid \tau \in S_{\prec}(\pi, \delta)\right\}_{\text {multi }} \\
& =\left\{\text { st } \tau \mid \tau \in S_{\prec}\left(\pi^{\prime}, \delta\right)\right\}_{\text {multi }} \\
& =\left\{\text { st } \tau \mid \tau \in S_{\prec}\left(\pi^{\prime}, \sigma^{\prime}\right)\right\}_{\text {multi }}
\end{aligned}
$$

The same argument (but with the symbols " $S_{\prec}$ " and " $S_{\succ}$ " interchanged) yields

$$
\left\{\text { st } \tau \mid \tau \in S_{\succ}(\pi, \sigma)\right\}_{\text {multi }}=\left\{\text { st } \tau \mid \tau \in S_{\succ}\left(\pi^{\prime}, \sigma^{\prime}\right)\right\}_{\text {multi }} .
$$

Thus, Claim 3 is proven in Case 2 .

We have now proven Claim 3 in each of the two Cases 1 and 2. Hence, Claim 3 always holds.]

Claim 3 says that for any two disjoint nonempty permutations $\pi$ and $\sigma$, the multisets

$$
\left\{\text { st } \tau \mid \tau \in S_{\prec}(\pi, \sigma)\right\}_{\text {multi }} \quad \text { and } \quad\left\{\text { st } \tau \mid \tau \in S_{\succ}(\pi, \sigma)\right\}_{\text {multi }}
$$

depend only on st $\pi$, st $\sigma,|\pi|,|\sigma|$ and $\left[\pi_{1}>\sigma_{1}\right]$. In other words, the statistic st is LRshuffle-compatible (by the definition of "LR-shuffle-compatible"). This proves Theorem 72 .

Combining Theorem 72 with Proposition 71, we obtain the following:

\section{Theorem 75 .}

(a) The permutation statistic Des is LR-shuffle-compatible.

(b) The permutation statistic Lpk is LR-shuffle-compatible.

(c) The permutation statistic Epk is LR-shuffle-compatible.

Proof of Theorem 75. 
(a) The permutation statistic Des is shuffle-compatible (by $[4, \S 2.4]$ ) and head-graftcompatible (by Proposition 71 (a)). Thus, Theorem 72 (applied to st $=$ Des) shows that the permutation statistic Des is LR-shuffle-compatible. This proves Theorem 75 (a).

(b) The permutation statistic Lpk is shuffle-compatible (by [4, Theorem 4.9 (a)]) and head-graft-compatible (by Proposition 71 (b)). Thus, Theorem 72 (applied to st $=$ Lpk) shows that the permutation statistic Lpk is LR-shuffle-compatible. This proves Theorem 75 (b).

(c) The permutation statistic Epk is shuffle-compatible (by Theorem 60) and headgraft-compatible (by Proposition 71 (c)). Thus, Theorem 72 (applied to st $=$ Epk) shows that the permutation statistic Epk is LR-shuffle-compatible. This proves Theorem 75 (c).

\subsection{Some other statistics}

The question of LR-shuffle-compatibility can be asked about any statistic. We have so far answered it for Des, Pk, Lpk, Rpk and Epk. In this section, we shall analyze it for some further statistics.

\subsubsection{The descent number des}

The permutation statistic des (called the descent number) is defined as follows: For each permutation $\pi$, we set $\operatorname{des} \pi=|\operatorname{Des} \pi|$ (that is, des $\pi$ is the number of all descents of $\pi$ ). It was proven in $[4$, Theorem 4.6 (a)] that this statistic des is shuffle-compatible. We now claim the following:

Proposition 76. The permutation statistic des is head-graft-compatible and LR-shufflecompatible.

Proof of Proposition 76. From (12), we easily obtain the following: If $\pi$ is a nonempty permutation, and if $a$ is a letter that does not appear in $\pi$, then

$$
\operatorname{des}(a: \pi)=\operatorname{des} \pi+\left[a>\pi_{1}\right] .
$$

Thus, des $(a: \pi)$ depends only on $\operatorname{des} \pi,|\pi|$ and $\left[a>\pi_{1}\right]$. In other words, des is head-graftcompatible (by the definition of "head-graft-compatible"). Hence, Theorem 72 (applied to st $=$ des) shows that the permutation statistic des is LR-shuffle-compatible. This proves Proposition 76 .

\subsubsection{The major index maj}

The permutation statistic maj (called the major index) is defined as follows: For each permutation $\pi$, we set maj $\pi=\sum_{i \in \operatorname{Des} \pi} i$ (that is, maj $\pi$ is the sum of all descents of $\pi$ ). It was proven in $[4$, Theorem 3.1 (a)] that this statistic maj is shuffle-compatible. 
However, maj is neither head-graft-compatible nor LR-shuffle-compatible. For example, if we take $\pi=(5,4,2,3), a=1, \pi^{\prime}=(3,4,5,2)$ and $a^{\prime}=1$, then we do have

$$
\operatorname{maj} \pi=\operatorname{maj}\left(\pi^{\prime}\right), \quad|\pi|=\left|\pi^{\prime}\right| \quad \text { and } \quad\left[a>\pi_{1}\right]=\left[a^{\prime}>\pi_{1}^{\prime}\right]
$$

but we don't have maj $(a: \pi)=$ maj $\left(a^{\prime}: \pi^{\prime}\right)$. Thus, maj is not head-graft-compatible. Using Corollary 81 below, this entails that maj is not LR-shuffle-compatible.

\subsubsection{The joint statistic (des, maj)}

The next permutation statistic we shall study is the so-called joint statistic (des, maj). This statistic is defined as the permutation statistic that sends each permutation $\pi$ to the ordered pair (des $\pi, \operatorname{maj} \pi)$. (Calling it (des, maj) is thus a slight abuse of notation.) It was proven in $[4$, Theorem 4.5 (a)] that this statistic (des, maj) is shuffle-compatible. We now claim the following:

Proposition 77. The permutation statistic (des, maj) is head-graft-compatible and LRshuffle-compatible.

Proof of Proposition 77. From (12), we easily obtain the following: If $\pi$ is a nonempty permutation, and if $a$ is a letter that does not appear in $\pi$, then

$$
\begin{aligned}
\operatorname{des}(a: \pi) & =\operatorname{des} \pi+\left[a>\pi_{1}\right] \quad \text { and } \\
\operatorname{maj}(a: \pi) & =\operatorname{des} \pi+\operatorname{maj} \pi+\left[a>\pi_{1}\right] .
\end{aligned}
$$

Thus, (des, maj) ( $a: \pi)$ depends only on (des, maj) $(\pi),|\pi|$ and $\left[a>\pi_{1}\right]$. In other words, (des, maj) is head-graft-compatible (by the definition of "head-graft-compatible"). Hence, Theorem 72 (applied to $s t=($ des, maj)) shows that the permutation statistic (des, maj) is LR-shuffle-compatible. This proves Proposition 77.

\subsubsection{The comajor index comaj}

The permutation statistic comaj (called the comajor index) is defined as follows: For each permutation $\pi$, we set $\operatorname{comaj} \pi=\sum_{k \in \operatorname{Des} \pi}(n-k)$, where $n=|\pi|$. It was proven in $[4, \S 3.2]$ that this statistic comaj is shuffle-compatible. We now claim the following:

Proposition 78. The permutation statistic comaj is head-graft-compatible and LR-shuffle-compatible.

Proof of Proposition 78. From (12), we easily obtain the following: If $\pi$ is a nonempty permutation, and if $a$ is a letter that does not appear in $\pi$, then

$$
\operatorname{comaj}(a: \pi)=\operatorname{comaj} \pi+\left[a>\pi_{1}\right] \cdot|\pi| \text {. }
$$

Thus, comaj $(a: \pi)$ depends only on $\operatorname{comaj} \pi,|\pi|$ and $\left[a>\pi_{1}\right]$. In other words, comaj is head-graft-compatible (by the definition of "head-graft-compatible"). Hence, Theorem 72 (applied to st $=$ comaj) shows that the permutation statistic comaj is LR-shufflecompatible. This proves Proposition 78. 


\subsection{Left- and right-shuffle-compatibility}

In this section, we shall study two notions closely related to LR-shuffle-compatibility:

Definition 79. Let st be a permutation statistic.

(a) We say that st is left-shuffle-compatible if for any two disjoint nonempty permutations $\pi$ and $\sigma$ having the property that $\pi_{1}>\sigma_{1}$, the multiset $\left\{\text { st } \tau \mid \tau \in S_{\prec}(\pi, \sigma)\right\}_{\text {multi }}$ depends only on st $\pi$, st $\sigma,|\pi|$ and $|\sigma|$.

(b) We say that st is right-shuffle-compatible if for any two disjoint nonempty permutations $\pi$ and $\sigma$ having the property that $\pi_{1}>\sigma_{1}$, the multiset $\left\{\text { st } \tau \mid \tau \in S_{\succ}(\pi, \sigma)\right\}_{\text {multi }}$ depends only on st $\pi$, st $\sigma,|\pi|$ and $|\sigma|$.

For a shuffle-compatible permutation statistic, these two notions are equivalent to the notions of LR-shuffle-compatibility and head-graft-compatibility, as the following proposition reveals:

Proposition 80. Let st be a shuffle-compatible permutation statistic. Then, the following assertions are equivalent:

- Assertion $\mathcal{A}_{1}$ : The statistic st is LR-shuffle-compatible.

- Assertion $\mathcal{A}_{2}$ : The statistic st is left-shuffle-compatible.

- Assertion $\mathcal{A}_{3}$ : The statistic st is right-shuffle-compatible.

- Assertion $\mathcal{A}_{4}$ : The statistic st is head-graft-compatible.

Proof of Proposition 80. Omitted; see [7].

Note that on their own, the properties of left-shuffle-compatibility and right-shufflecompatibility are not equivalent. For example, the permutation statistic that sends each nonempty permutation $\pi$ to the truth value $\left[\pi_{1}>\pi_{i}\right.$ for all $\left.i>1\right]$ (and the 0 -permutation () to 0 ) is right-shuffle-compatible (because in the definition of right-shuffle-compatibility, all the st $\tau$ will be 0 ), but not left-shuffle-compatible.

\subsection{Properties of compatible statistics}

Let us state some more facts on compatibility properties. We refer to [7] for their proofs. We begin with a converse to Theorem 72 :

Corollary 81. Let st be a LR-shuffle-compatible permutation statistic. Then, st is shufflecompatible, left-shuffle-compatible, right-shuffle-compatible and head-graft-compatible.

Corollary 82. Let st be a permutation statistic that is left-shuffle-compatible and rightshuffle-compatible. Then, st is LR-shuffle-compatible. 


\section{Descent statistics and quasisymmetric functions}

In this section, we shall recall the concepts of descent statistics and their shuffle algebras (introduced in [4]), and apply them to Epk.

\subsection{Compositions}

Definition 83. A composition is a finite list of positive integers. If $I=\left(i_{1}, i_{2}, \ldots, i_{n}\right)$ is a composition, then the nonnegative integer $i_{1}+i_{2}+\cdots+i_{n}$ is called the size of $I$ and is denoted by $|I|$; we furthermore say that $I$ is a composition of $|I|$.

Definition 84. Let $n \in \mathbb{N}$. For each composition $I=\left(i_{1}, i_{2}, \ldots, i_{k}\right)$ of $n$, we define a subset Des $I$ of $[n-1]$ by

$$
\begin{aligned}
\operatorname{Des} I & =\left\{i_{1}, i_{1}+i_{2}, i_{1}+i_{2}+i_{3}, \ldots, i_{1}+i_{2}+\cdots+i_{k-1}\right\} \\
& =\left\{i_{1}+i_{2}+\cdots+i_{s} \mid s \in[k-1]\right\} .
\end{aligned}
$$

On the other hand, for each subset $A=\left\{a_{1}<a_{2}<\cdots<a_{k}\right\}$ of $[n-1]$, we define a composition Comp $A$ of $n$ by

$$
\operatorname{Comp} A=\left(a_{1}, a_{2}-a_{1}, a_{3}-a_{2}, \ldots, a_{k}-a_{k-1}, n-a_{k}\right) .
$$

(The definition of Comp $A$ should be understood to give Comp $A=(n)$ if $A=\varnothing$ and $n>0$, and to give $\operatorname{Comp} A=()$ if $A=\varnothing$ and $n=0$. Note that Comp $A$ depends not only on the set $A$ itself, but also on $n$. We hope that $n$ will always be clear from the context when we use this notation.)

We thus have defined a map Des (from the set of all compositions of $n$ to the set of all subsets of $[n-1]$ ) and a map Comp (in the opposite direction). These two maps are mutually inverse bijections.

Definition 85. Let $n \in \mathbb{N}$. Let $\pi=\left(\pi_{1}, \pi_{2}, \ldots, \pi_{n}\right)$ be an $n$-permutation. The descent composition of $\pi$ is defined to be the composition Comp (Des $\pi$ ) of $n$. This composition is denoted by $\operatorname{Comp} \pi$.

For example, the 6-permutation $\pi=(4,1,3,9,6,8)$ has $\operatorname{Comp} \pi=(1,3,2)$. For another example, the 6-permutation $\pi=(1,4,3,2,9,8)$ has $\operatorname{Comp} \pi=(2,1,2,1)$.

Definition 85 defines the permutation statistic Comp, whose codomain is the set of all compositions.

\subsection{Descent statistics}

Definition 86. Let st be a permutation statistic. We say that st is a descent statistic if and only if st $\pi$ (for $\pi$ a permutation) depends only on the descent composition Comp $\pi$ of $\pi$. In other words, st is a descent statistic if and only if every two permutations $\pi$ and $\sigma$ satisfying $\operatorname{Comp} \pi=\operatorname{Comp} \sigma$ satisfy st $\pi=\operatorname{st} \sigma$. 
Equivalently, a permutation statistic st is a descent statistic if and only if every two permutations $\pi$ and $\sigma$ satisfying $|\pi|=|\sigma|$ and $\operatorname{Des} \pi=\operatorname{Des} \sigma$ satisfy st $\pi=\operatorname{st} \sigma$. (This is indeed equivalent, because for two permutations $\pi$ and $\sigma$, the condition $(|\pi|=|\sigma|$ and $\operatorname{Des} \pi=\operatorname{Des} \sigma)$ is equivalent to $(\operatorname{Comp} \pi=\operatorname{Comp} \sigma)$.)

For example, the permutation statistic Des is a descent statistic, because each permutation $\pi$ satisfies Des $\pi=\operatorname{Des}(\operatorname{Comp} \pi)$. Also, $\mathrm{Pk}$ is a descent statistic, since each permutation $\pi$ satisfies

$$
\operatorname{Pk} \pi=(\operatorname{Des} \pi) \backslash(\{1\} \cup(\operatorname{Des} \pi+1)),
$$

where Des $\pi+1$ denotes the set $\{i+1 \mid i \in \operatorname{Des} \pi\}$ (and, as we have just said, Des $\pi$ can be recovered from Comp $\pi$ ). Furthermore, Epk is a descent statistic, since each $n$ permutation $\pi$ (for a positive integer $n$ ) satisfies

$$
\operatorname{Epk} \pi=(\operatorname{Des} \pi \cup\{n\}) \backslash(\operatorname{Des} \pi+1)
$$

(and both Des $\pi$ and $n$ can be recovered from Comp $\pi$ ). The permutation statistics Lpk and Rpk (and, of course, Comp) are descent statistics as well, as one can easily check.

In [10, Corollary 1.6], Ezgi Kantarcı Oğuz has demonstrated that not every shufflecompatible permutation statistic is a descent statistic. However, this changes if we require LR-shuffle-compatibility, because of Corollary 81 and of the following fact:

Proposition 87. Every head-graft-compatible permutation statistic is a descent statistic.

Proof of Proposition 8\%. See [7].

Definition 88. Let st be a descent statistic. Then, we can regard st as a map from the set of all compositions (rather than from the set of all permutations). Namely, for any composition $I$, we define st $I$ (an element of the codomain of st) by setting

$$
\text { st } I=\text { st } \pi \quad \text { for any permutation } \pi \text { satisfying } \operatorname{Comp} \pi=I .
$$

This is well-defined (because for every composition $I$, there exists at least one permutation $\pi$ satisfying Comp $\pi=I$, and all such permutations $\pi$ have the same value of st $\pi$ ). In the following, we shall regard every descent statistic st simultaneously as a map from the set of all permutations and as a map from the set of all compositions.

Note that this definition leads to a new interpretation of Des $I$ for a composition $I$ : It is now defined as Des $\pi$ for any permutation $\pi$ satisfying $\operatorname{Comp} \pi=I$. This could clash with the old meaning of Des $I$ introduced in Definition 84. Fortunately, these two meanings of Des $I$ are exactly the same, so there is no conflict of notation.

However, Definition 88 causes an ambiguity for expressions like "Des $\left(i_{1}, i_{2}, \ldots, i_{n}\right)$ ": Here, the " $\left(i_{1}, i_{2}, \ldots, i_{n}\right)$ " might be understood either as a permutation, or as a composition, and the resulting descent sets $\operatorname{Des}\left(i_{1}, i_{2}, \ldots, i_{n}\right)$ are not the same. A similar ambiguity occurs for any descent statistic st instead of Des. We hope that this ambiguity will not arise in this paper due to our explicit typecasting of permutations and compositions; but the reader should be warned that it can arise if one takes the notation too literally. 
Definition 89. Let st be a descent statistic.

(a) Two compositions $J$ and $K$ are said to be st-equivalent if and only if they have the same size and satisfy st $J=$ st $K$. Equivalently, two compositions $J$ and $K$ are st-equivalent if and only if there exist two st-equivalent permutations $\pi$ and $\sigma$ satisfying $J=\operatorname{Comp} \pi$ and $K=\operatorname{Comp} \sigma$.

(b) The relation "st-equivalent" is an equivalence relation on compositions; its equivalence classes are called st-equivalence classes of compositions.

\subsection{Quasisymmetric functions}

We now recall the definition of quasisymmetric functions; see [8, Chapter 5] (and various other modern textbooks) for more details about this:

\section{Definition 90.}

(a) Consider the ring of power series $\mathbb{Q}\left[\left[x_{1}, x_{2}, x_{3}, \ldots\right]\right]$ in infinitely many commuting indeterminates over $\mathbb{Q}$. A power series $f \in \mathbb{Q}\left[\left[x_{1}, x_{2}, x_{3}, \ldots\right]\right]$ is said to be quasisymmetric if it has the following property:

- For any positive integers $a_{1}, a_{2}, \ldots, a_{k}$ and any two strictly increasing sequences $\left(i_{1}<i_{2}<\cdots<i_{k}\right)$ and $\left(j_{1}<j_{2}<\cdots<j_{k}\right)$ of positive integers, the coefficient of $x_{i_{1}}^{a_{1}} x_{i_{2}}^{a_{2}} \cdots x_{i_{k}}^{a_{k}}$ in $f$ equals the coefficient of $x_{j_{1}}^{a_{1}} x_{j_{2}}^{a_{2}} \cdots x_{j_{k}}^{a_{k}}$ in $f$.

(b) A quasisymmetric function is a quasisymmetric power series $f \in \mathbb{Q}\left[\left[x_{1}, x_{2}, x_{3}, \ldots\right]\right]$ that has bounded degree (i.e., there exists an $N \in \mathbb{N}$ such that each monomial appearing in $f$ has degree $\leqslant N)$.

(c) The quasisymmetric functions form a $\mathbb{Q}$-subalgebra of $\mathbb{Q}\left[\left[x_{1}, x_{2}, x_{3}, \ldots\right]\right]$; this $\mathbb{Q}$ subalgebra is denoted by QSym and called the ring of quasisymmetric functions over $\mathbb{Q}$. This $\mathbb{Q}$-algebra QSym is graded (in the obvious way, i.e., by the degree of a monomial).

The $\mathbb{Q}$-algebra QSym has much interesting structure (e.g., it is a Hopf algebra), some of which we will introduce later when we need it. One simple yet crucial feature of QSym that we will immediately use is the fundamental basis of QSym:

Definition 91. For any composition $\alpha$, we define the fundamental quasisymmetric function $F_{\alpha}$ to be the power series

$$
\sum_{\substack{i_{1} \leqslant i_{2} \leqslant \cdots \leqslant i_{n} ; \\ i_{j}<i_{j+1} \text { for each } j \in \operatorname{Des} \alpha}} x_{i_{1}} x_{i_{2}} \cdots x_{i_{n}} \in \text { QSym, }
$$

where $n=|\alpha|$ is the size of $\alpha$. The family $\left(F_{\alpha}\right)_{\alpha}$ is a composition is a basis of the $\mathbb{Q}$-vector space QSym; it is known as the fundamental basis of QSym. 
The fundamental quasisymmetric function $F_{\alpha}$ is denoted by $L_{\alpha}$ in $[8, \S 5.2]$.

The multiplication of fundamental quasisymmetric functions is intimately related to shuffles of permutations:

Proposition 92. Let $\pi$ and $\sigma$ be two disjoint permutations. Then,

$$
F_{\mathrm{Comp} \pi} F_{\mathrm{Comp} \sigma}=\sum_{\chi \in S(\pi, \sigma)} F_{\mathrm{Comp} \chi}
$$

Proposition 92 is a restatement of [4, Theorem 4.1], and is proven in [8, (5.2.6)] (which makes the additional requirement that the letters of $\pi$ are $1,2, \ldots,|\pi|$ and the letters of $\sigma$ are $|\pi|+1,|\pi|+2, \ldots,|\pi|+|\sigma|$; but this requirement is not used in the proof and thus can be dropped).

\subsection{Shuffle algebras}

Any shuffle-compatible permutation statistic st gives rise to a shuffle algebra $\mathcal{A}_{\text {st }}$, defined as follows:

Definition 93. Let st be a shuffle-compatible permutation statistic. For each permutation $\pi$, let $[\pi]_{\text {st }}$ denote the st-equivalence class of $\pi$.

Let $\mathcal{A}_{\mathrm{st}}$ be the free $\mathbb{Q}$-vector space whose basis is the set of all st-equivalence classes of permutations. We define a multiplication on $\mathcal{A}_{\text {st }}$ by setting

$$
[\pi]_{\mathrm{st}}[\sigma]_{\mathrm{st}}=\sum_{\tau \in S(\pi, \sigma)}[\tau]_{\mathrm{st}}
$$

for any two disjoint permutations $\pi$ and $\sigma$. It is easy to see that this multiplication is welldefined and associative, and turns $\mathcal{A}_{\text {st }}$ into a $\mathbb{Q}$-algebra whose unity is the st-equivalence class of the 0 -permutation (). This $\mathbb{Q}$-algebra is denoted by $\mathcal{A}_{\text {st }}$, and is called the shuffle algebra of st. It is a graded $\mathbb{Q}$-algebra; its $n$-th graded component (for each $n \in \mathbb{N}$ ) is spanned by the st-equivalence classes of all $n$-permutations.

This definition originates in $[4, \S 3.1]$. The following fact is implicit in [4]:

Proposition 94. Let st be a shuffle-compatible descent statistic.

There is a surjective $\mathbb{Q}$-algebra homomorphism $p_{\mathrm{st}}:$ QSym $\rightarrow \mathcal{A}_{\mathrm{st}}$ that satisfies

$$
p_{\mathrm{st}}\left(F_{\mathrm{Comp} \pi}\right)=[\pi]_{\mathrm{st}} \quad \text { for every permutation } \pi .
$$

A central result, connecting shuffle-compatibility of a descent statistic with QSym, is [4, Theorem 4.3], which we restate as follows: 
Theorem 95. Let st be a descent statistic.

(a) The descent statistic st is shuffle-compatible if and only if there exist a $\mathbb{Q}$-algebra A with basis $\left(u_{\alpha}\right)$ (indexed by st-equivalence classes $\alpha$ of compositions) and a $\mathbb{Q}$ algebra homomorphism $\phi_{\mathrm{st}}:$ QSym $\rightarrow A$ with the property that whenever $\alpha$ is an st-equivalence class of compositions, we have

$$
\phi_{\text {st }}\left(F_{L}\right)=u_{\alpha} \quad \text { for each } L \in \alpha .
$$

(b) In this case, the $\mathbb{Q}$-linear map

$$
\mathcal{A}_{\mathrm{st}} \rightarrow A, \quad[\pi]_{\mathrm{st}} \mapsto u_{\alpha},
$$

where $\alpha$ is the st-equivalence class of the composition $\operatorname{Comp} \pi$, is a $\mathbb{Q}$-algebra isomorphism $\mathcal{A}_{\text {st }} \rightarrow A$.

Proofs of Proposition 94 and Theorem 95 (independent of [4]) can be found in [7].

\subsection{The shuffle algebra of Epk}

Theorem 60 yields that the permutation statistic Epk is shuffle-compatible. Hence, the shuffle algebra $\mathcal{A}_{\mathrm{Epk}}$ is well-defined. We have little to say about it:

\section{Theorem 96.}

(a) The shuffle algebra $\mathcal{A}_{\mathrm{Epk}}$ is a graded quotient algebra of QSym.

(b) Define the Fibonacci sequence $\left(f_{0}, f_{1}, f_{2}, \ldots\right)$ as in Proposition 15. Let $n$ be a positive integer. The $n$-th graded component of $\mathcal{A}_{\mathrm{Epk}}$ has dimension $f_{n+2}-1$.

Proof of Theorem 96. See [7].

We can describe $\mathcal{A}_{\mathrm{Epk}}$ using the notations of Section 2:

Definition 97. Let $\Pi_{\mathcal{Z}}$ be the $\mathbb{Q}$-vector subspace of Pow $\mathcal{N}$ spanned by the family $\left(K_{n, \Lambda}^{\mathcal{Z}}\right)_{n \in \mathbb{N} ; \Lambda \in \mathbf{L}_{n}}$. Then, $\Pi_{\mathcal{Z}}$ is also the $\mathbb{Q}$-vector subspace of Pow $\mathcal{N}$ spanned by the family $\left(K_{n, \operatorname{Epk} \pi}^{\mathcal{Z}}\right)_{n \in \mathbb{N} ; \pi \text { is an } n \text {-permutation }}$ (by Proposition 17). In other words, $\Pi_{\mathcal{Z}}$ is also the $\mathbb{Q}$-vector subspace of Pow $\mathcal{N}$ spanned by the family $\left(\Gamma_{\mathcal{Z}}(\pi)\right)_{n \in \mathbb{N} ; \pi}$ is an $n$-permutation (because of (8)). Hence, Corollary 41 shows that $\Pi_{\mathcal{Z}}$ is closed under multiplication. Since furthermore $\Gamma_{\mathcal{Z}}(())=1$ (for the 0-permutation () ), we can thus conclude that $\Pi_{\mathcal{Z}}$ is a $\mathbb{Q}$-subalgebra of Pow $\mathcal{N}$.

Theorem 98. The $\mathbb{Q}$-linear map

$$
\mathcal{A}_{\mathrm{Epk}} \rightarrow \Pi_{\mathcal{Z}}, \quad[\pi]_{\mathrm{Epk}} \mapsto K_{n, \mathrm{Epk} \pi}^{\mathcal{Z}}
$$

is a $\mathbb{Q}$-algebra isomorphism.

Proof of Theorem 98. See [7]. 


\section{The kernel of the map QSym $\rightarrow \mathcal{A}_{\mathrm{Epk}}$}

\subsection{The kernel of a descent statistic}

Now, we shall focus on a feature of shuffle-compatible descent statistics that seems to have been overlooked so far: their kernels.

All proofs in this section are omitted; they can be found in [7].

Definition 99. Let st be a descent statistic. Then, $\mathcal{K}_{\text {st }}$ shall mean the $\mathbb{Q}$-vector subspace of QSym spanned by all elements of the form $F_{J}-F_{K}$, where $J$ and $K$ are two st-equivalent compositions. (See Definition 89 (a) for the definition of "st-equivalent compositions".) We shall refer to $\mathcal{K}_{\text {st }}$ as the kernel of st.

The following basic linear-algebraic lemma will be useful:

Lemma 100. Let st be a descent statistic. Let $A$ be a $\mathbb{Q}$-vector space with basis $\left(u_{\alpha}\right)$ indexed by st-equivalence classes $\alpha$ of compositions. Let $\phi_{\mathrm{st}}$ : QSym $\rightarrow A$ be a $\mathbb{Q}$-linear map with the property that whenever $\alpha$ is an st-equivalence class of compositions, we have

$$
\phi_{\text {st }}\left(F_{L}\right)=u_{\alpha} \quad \text { for each } L \in \alpha .
$$

Then, $\operatorname{Ker}\left(\phi_{\text {st }}\right)=\mathcal{K}_{\text {st }}$.

Theorem 95 easily yields the following fact:

Proposition 101. Let st be a descent statistic. Then, st is shuffle-compatible if and only if $\mathcal{K}_{\mathrm{st}}$ is an ideal of QSym. Furthermore, in this case, $\mathcal{A}_{\mathrm{st}} \cong \mathrm{QSym} / \mathcal{K}_{\mathrm{st}}$ as $\mathbb{Q}$-algebras.

Corollary 102. The kernel $\mathcal{K}_{\mathrm{Epk}}$ of the descent statistic Epk is an ideal of QSym.

We can study the kernel of any descent statistic; in particular, the case of shufflecompatible descent statistics appears interesting. Since QSym is isomorphic to a polynomial ring (as an algebra), it has many ideals, which are rather hopeless to classify or tame; but the ones obtained as kernels of shuffle-compatible descent statistics might be worth discussing.

\subsection{An F-generating set of $\mathcal{K}_{\mathrm{Epk}}$}

Let us now focus on $\mathcal{K}_{\text {Epk }}$, the kernel of Epk.

Proposition 103. If $J=\left(j_{1}, j_{2}, \ldots, j_{m}\right)$ and $K$ are two compositions, then we shall write $J \rightarrow K$ if there exists an $\ell \in\{2,3, \ldots, m\}$ such that $j_{\ell}>2$ and

$$
K=\left(j_{1}, j_{2}, \ldots, j_{\ell-1}, 1, j_{\ell}-1, j_{\ell+1}, j_{\ell+2}, \ldots, j_{m}\right) .
$$

(In other words, we write $J \rightarrow K$ if $K$ can be obtained from $J$ by "splitting" some entry $j_{\ell}>2$ into two consecutive entries 1 and $j_{\ell}-1$, provided that this entry was not the first entry - i.e., we had $\ell>1$ - and that this entry was greater than 2.)

The ideal $\mathcal{K}_{\mathrm{Epk}}$ of QSym is spanned (as a $\mathbb{Q}$-vector space) by all differences of the form $F_{J}-F_{K}$, where $J$ and $K$ are two compositions satisfying $J \rightarrow K$. 
Example 104. We have $(2,1,4,4) \rightarrow(2,1,1,3,4)$, since the composition $(2,1,1,3,4)$ is obtained from $(2,1,4,4)$ by splitting the third entry (which is $4>2$ ) into two consecutive entries 1 and 3 .

Similarly, $(2,1,4,4) \rightarrow(2,1,4,1,3)$.

But we do not have $(3,1) \rightarrow(1,2,1)$, because splitting the first entry of the composition is not allowed in the definition of the relation $\rightarrow$. Also, we do not have $(1,2,1) \rightarrow$ $(1,1,1,1)$, because the entry we are splitting must be $>2$.

Two compositions $J$ and $K$ satisfying $J \rightarrow K$ must necessarily satisfy $|J|=|K|$.

Here are all relations $\rightarrow$ between compositions of size 4 :

$$
(1,3) \rightarrow(1,1,2) .
$$

Here are all relations $\rightarrow$ between compositions of size $5:$

$$
\begin{aligned}
(1,4) & \rightarrow(1,1,3), \\
(1,3,1) & \rightarrow(1,1,2,1), \\
(1,1,3) & \rightarrow(1,1,1,2), \\
(2,3) & \rightarrow(2,1,2) .
\end{aligned}
$$

There are no relations $\rightarrow$ between compositions of size $\leqslant 3$.

\subsection{An M-generating set of $\mathcal{K}_{\mathrm{Epk}}$}

Another characterization of the ideal $\mathcal{K}_{\text {Epk }}$ of QSym can be obtained using the monomial basis of QSym. Let us first recall how said basis is defined:

For any composition $\alpha=\left(\alpha_{1}, \alpha_{2}, \ldots, \alpha_{\ell}\right)$, we let

$$
M_{\alpha}=\sum_{i_{1}<i_{2}<\cdots<i_{\ell}} x_{i_{1}}^{\alpha_{1}} x_{i_{2}}^{\alpha_{2}} \cdots x_{i_{\ell}}^{\alpha_{\ell}}
$$

(where the sum is over all strictly increasing $\ell$-tuples $\left(i_{1}, i_{2}, \ldots, i_{\ell}\right)$ of positive integers). This power series $M_{\alpha}$ belongs to QSym. The family $\left(M_{\alpha}\right)_{\alpha}$ is a composition is a basis of the $\mathbb{Q}$-vector space QSym; it is called the monomial basis of QSym.

Proposition 105. If $J=\left(j_{1}, j_{2}, \ldots, j_{m}\right)$ and $K$ are two compositions, then we shall write $J \underset{M}{\rightarrow} K$ if there exists an $\ell \in\{2,3, \ldots, m\}$ such that $j_{\ell}>2$ and

$$
K=\left(j_{1}, j_{2}, \ldots, j_{\ell-1}, 2, j_{\ell}-2, j_{\ell+1}, j_{\ell+2}, \ldots, j_{m}\right)
$$

(In other words, we write $J \underset{M}{\rightarrow} K$ if $K$ can be obtained from $J$ by "splitting" some entry $j_{\ell}>2$ into two consecutive entries 2 and $j_{\ell}-2$, provided that this entry was not the first entry - i.e., we had $\ell>1$ - and that this entry was greater than 2.)

The ideal $\mathcal{K}_{\mathrm{Epk}}$ of QSym is spanned (as a $\mathbb{Q}$-vector space) by all sums of the form $M_{J}+M_{K}$, where $J$ and $K$ are two compositions satisfying $J \underset{M}{\rightarrow} K$.

\footnotetext{
${ }^{7}$ The word "consecutive" here means "in consecutive positions of $J$ ", not "consecutive integers". So two consecutive entries of $J$ are two entries of the form $j_{p}$ and $j_{p+1}$ for some $p \in\{1,2, \ldots, m-1\}$.
} 
Example 106. We have $(2,1,4,4) \underset{M}{\rightarrow}(2,1,2,2,4)$, since the composition $(2,1,2,2,4)$ is obtained from $(2,1,4,4)$ by splitting the third entry (which is $4>2$ ) into two consecutive entries 2 and 2 .

Similarly, $(2,1,4,4) \underset{M}{\rightarrow}(2,1,4,2,2)$ and $(2,1,5,4) \underset{M}{\rightarrow}(2,1,2,3,4)$.

But we do not have $(3,1) \underset{M}{\rightarrow}(2,1,1)$, because splitting the first entry of the composition is not allowed in the definition of the relation $\underset{M}{\rightarrow}$.

Two compositions $J$ and $K$ satisfying $J \underset{M}{\rightarrow} K$ must necessarily satisfy $|J|=|K|$.

Here are all relations $\underset{M}{\rightarrow}$ between compositions of size 4 :

$$
(1,3) \underset{M}{\rightarrow}(1,2,1) .
$$

Here are all relations $\underset{M}{\rightarrow}$ between compositions of size 5:

$$
\begin{gathered}
(1,4) \underset{M}{\rightarrow}(1,2,2), \\
(1,3,1) \underset{M}{\rightarrow}(1,2,1,1), \\
(1,1,3) \underset{M}{\rightarrow}(1,1,2,1), \\
(2,3) \underset{M}{\rightarrow}(2,2,1) .
\end{gathered}
$$

There are no relations $\underset{M}{\rightarrow}$ between compositions of size $\leqslant 3$.

Question 107. It is worth analyzing the kernels of other known descent statistics (shufflecompatible or not). Let us say that a descent statistic st is $M$-binomial if its kernel $\mathcal{K}_{\text {st }}$ can be spanned by elements of the form $\lambda M_{J}+\mu M_{K}$ with $\lambda, \mu \in \mathbb{Q}$ and compositions $J, K$. Then, Proposition 105 yields that Epk is M-binomial. It is easy to see that the statistics Des and des are M-binomial as well. Computations using SageMath suggest that the statistics Lpk, Rpk, Pk, Val, pk, lpk, rpk and val (see [4] for some of their definitions) are M-binomial, too (at least for compositions of size $\leqslant 9$ ); this would be nice to prove. On the other hand, the statistics maj, (des, maj) and (val, des) (again, see [4] for definitions) are not M-binomial.

\section{Dendriform structures}

Next, we shall recall the dendriform operations $\prec$ and $\succeq$ on QSym studied in [6], and we shall connect these operations back to LR-shuffle-compatibility. Since we consider this somewhat tangential to the present paper, we merely summarize the main results here; more can be found in [7].

\subsection{Two operations on QSym}

We begin with some definitions. We will use some notations from $[6]$, but we set $\mathbf{k}=\mathbb{Q}$ because we are working over the ring $\mathbb{Q}$ in this paper. Monomials always mean formal 
expressions of the form $x_{1}^{a_{1}} x_{2}^{a_{2}} x_{3}^{a_{3}} \cdots$ with $a_{1}+a_{2}+a_{3}+\cdots<\infty$ (see [6, Section 2] for details). If $\mathfrak{m}$ is a monomial, then Supp $\mathfrak{m}$ will denote the finite subset

$$
\left\{i \in\{1,2,3, \ldots\} \mid \text { the exponent with which } x_{i} \text { occurs in } \mathfrak{m} \text { is }>0\right\}
$$

of $\{1,2,3, \ldots\}$. Next, we define two binary operations

$\prec$ (called "dendriform less-than"; but it's an operation, not a relation),

$\succeq$ (called "dendriform greater-or-equal"; but it's an operation, not a relation),

on the ring $\mathbf{k}\left[\left[x_{1}, x_{2}, x_{3}, \ldots\right]\right]$ of power series by first defining how they act on monomials:

$$
\begin{aligned}
& \mathfrak{m} \prec \mathfrak{n}=\left\{\begin{array}{lr}
\mathfrak{m} \cdot \mathfrak{n}, & \text { if } \min (\operatorname{Supp} \mathfrak{m})<\min (\operatorname{Supp} \mathfrak{n}) ; \\
0, & \text { if } \min (\operatorname{Supp} \mathfrak{m}) \geqslant \min (\operatorname{Supp} \mathfrak{n})
\end{array} ;\right. \\
& \mathfrak{m} \succeq \mathfrak{n}=\left\{\begin{array}{lr}
\mathfrak{m} \cdot \mathfrak{n}, & \text { if } \min (\operatorname{Supp} \mathfrak{m}) \geqslant \min (\operatorname{Supp} \mathfrak{n}) ; \\
0, & \text { if } \min (\operatorname{Supp} \mathfrak{m})<\min (\operatorname{Supp} \mathfrak{n})
\end{array} ;\right.
\end{aligned}
$$

and then requiring that they all be $\mathbf{k}$-bilinear and continuous (so their action on pairs of arbitrary power series can be computed by "opening the parentheses"). These operations $\prec$ and $\succeq$ restrict to the subset QSym of $\mathbf{k}\left[\left[x_{1}, x_{2}, x_{3}, \ldots\right]\right]$ (this is proven in [6, detailed version, Section 3]). They furthermore satisfy the following relations (which are easy to verify):

- For all $a, b, c \in \mathbf{k}\left[\left[x_{1}, x_{2}, x_{3}, \ldots\right]\right]$, we have

$$
\begin{aligned}
a \prec b+a \succeq b & =a b ; \\
(a \prec b) \prec c & =a \prec(b c) ; \quad(a \succeq b) \prec c=a \succeq(b \prec c) ; \\
a \succeq(b \succeq c) & =(a b) \succeq c .
\end{aligned}
$$

- For any $a \in \mathbf{k}\left[\left[x_{1}, x_{2}, x_{3}, \ldots\right]\right]$, we have

$$
1 \prec a=0 ; \quad a \prec 1=a-\varepsilon(a) ; \quad 1 \succeq a=a ; \quad a \succeq 1=\varepsilon(a),
$$

where $\varepsilon(a)$ denotes the constant term of the power series $a$.

The operations $\prec$ and $\succeq$ are sometimes called "restricted products" due to their similarity with the (regular) multiplication of QSym. In particular, they satisfy the following analogue of Proposition 92:

Proposition 108. Let $\pi$ and $\sigma$ be two disjoint nonempty permutations. Assume that $\pi_{1}>\sigma_{1}$. Then,

$$
\begin{aligned}
& F_{\text {Comp } \pi} \prec F_{\text {Comp } \sigma}=\sum_{\chi \in S_{\prec}(\pi, \sigma)} F_{\text {Comp } \chi} \quad \text { and } \\
& F_{\text {Comp } \pi} \succeq F_{\text {Comp } \sigma}=\sum_{\chi \in S_{\succ}(\pi, \sigma)} F_{\mathrm{Comp} \chi} .
\end{aligned}
$$




\subsection{Left- and right-shuffle-compatibility and ideals}

This proposition lets us relate the notions introduced in Definition 79 to the operations $\prec$ and $\succeq$. To state the precise connection, we need the following notation:

Definition 109. Let $A$ be a k-module equipped with some binary operation $*$ (written infix).

(a) If $B$ and $C$ are two k-submodules of $A$, then $B * C$ shall mean the k-submodule of $A$ spanned by all elements of the form $b * c$ with $b \in B$ and $c \in C$.

(b) A k-submodule $M$ of $A$ is said to be a $*$-ideal if and only if it satisfies $A * M \subseteq M$ and $M * A \subseteq M$.

Now, let us define two further variants of LR-shuffle-compatibility (to be compared with those introduced in Definition 79):

Definition 110. Let st be a permutation statistic.

(a) We say that st is weakly left-shuffle-compatible if for any two disjoint nonempty permutations $\pi$ and $\sigma$ having the property that

each entry of $\pi$ is greater than each entry of $\sigma$,

the multiset $\left\{\text { st } \tau \mid \tau \in S_{\prec}(\pi, \sigma)\right\}_{\text {multi }}$ depends only on st $\pi$, st $\sigma,|\pi|$ and $|\sigma|$.

(b) We say that st is weakly right-shuffle-compatible if for any two disjoint nonempty permutations $\pi$ and $\sigma$ having the property that

each entry of $\pi$ is greater than each entry of $\sigma$,

the multiset $\left\{\operatorname{st} \tau \mid \tau \in S_{\succ}(\pi, \sigma)\right\}_{\text {multi }}$ depends only on st $\pi$, st $\sigma,|\pi|$ and $|\sigma|$.

Then, the following analogues to the first part of Proposition 101 hold:

Theorem 111. Let st be a descent statistic. Then, the following three statements are equivalent:

- Statement A: The statistic st is left-shuffle-compatible.

- Statement B: The statistic st is weakly left-shuffle-compatible.

- Statement $C$ : The set $\mathcal{K}_{\mathrm{st}}$ is an $\prec$-ideal of QSym.

Theorem 112. Let st be a descent statistic. Then, the following three statements are equivalent:

- Statement A: The statistic st is right-shuffle-compatible.

- Statement B: The statistic st is weakly right-shuffle-compatible.

- Statement $C$ : The set $\mathcal{K}_{\mathrm{st}}$ is an $\succeq$-ideal of QSym. 
Corollary 113. Let st be a permutation statistic that is LR-shuffle-compatible. Then, st is a shuffle-compatible descent statistic, and the set $\mathcal{K}_{\mathrm{st}}$ is an ideal and $a \prec$-ideal and a $\succeq$-ideal of QSym.

Corollary 114. Let st be a descent statistic such that $\mathcal{K}_{\text {st }}$ is a $\prec$-ideal and a $\succeq$-ideal of QSym. Then, st is LR-shuffle-compatible and shuffle-compatible.

Corollary 113 can (for example) be applied to st = Epk, which we know to be LRshuffle-compatible (from Theorem 75 (c)); the result is that $\mathcal{K}_{\text {Epk }}$ is an ideal and a $\prec-$ ideal and $\mathrm{a} \succeq$-ideal of QSym. The same can be said about Des and Lpk and some other statistics.

Combining Theorem 111 with Theorem 112, we can also see that any descent statistic that is weakly left-shuffle-compatible and weakly right-shuffle-compatible must automatically be shuffle-compatible (because any $\prec$-ideal of QSym that is also a $\succeq$-ideal of QSym is an ideal of QSym as well). Note that this is only true for descent statistics! As far as arbitrary permutation statistics are concerned, this is false; for example, the number of inversions is weakly left-shuffle-compatible and weakly right-shuffle-compatible but not shuffle-compatible.

Let us next define the notion of dendriform algebras:

\section{Definition 115.}

(a) A dendriform algebra over a field $\mathbf{k}$ means a $\mathbf{k}$-algebra $A$ equipped with two further k-bilinear binary operations $\prec$ and $\succeq$ (these are operations, not relations, despite the symbols) from $A \times A$ to $A$ that satisfy the four rules

$$
\begin{aligned}
& a \prec b+a \succeq b=a b ; \\
& (a \prec b) \prec c=a \prec(b c) ; \\
& (a \succeq b) \prec c=a \succeq(b \prec c) ; \\
& a \succeq(b \succeq c)=(a b) \succeq c
\end{aligned}
$$

for all $a, b, c \in A$. (Depending on the situation, it is useful to also impose a few axioms that relate the unity 1 of the k-algebra $A$ with the operations $\prec$ and $\succeq$. For example, we could require $1 \prec a=0$ for each $a \in A$. For what we are going to do, these extra axioms don't matter.)

(b) If $A$ and $B$ are two dendriform algebras over $\mathbf{k}$, then a dendriform algebra homomorphism from $A$ to $B$ means a k-algebra homomorphism $\phi: A \rightarrow B$ preserving the operations $\prec$ and $\succeq$ (that is, satisfying $\phi(a \prec b)=\phi(a) \prec \phi(b)$ and $\phi(a \succeq b)=\phi(a) \succeq \phi(b)$ for all $a, b \in A$ ). (Some authors only require it to be a k-linear map instead of being a k-algebra homomorphism; this boils down to the question whether $\phi(1)$ must be 1 or not. This does not make a difference for us here.) 

$\mathbb{Q}$.

Thus, QSym (with its two operations $\prec$ and $\succeq$ ) becomes a dendriform algebra over

Notice that if $A$ and $B$ are two dendriform algebras over $\mathbf{k}$, then the kernel of any dendriform algebra homomorphism $A \rightarrow B$ is an $\prec$-ideal and a $\succeq$-ideal of $A$. Conversely, if $A$ is a dendriform algebra over $\mathbf{k}$, and $I$ is simultaneously a $\prec$-ideal and a $\succeq$-ideal of $A$, then $A / I$ canonically becomes a dendriform algebra, and the canonical projection $A \rightarrow A / I$ becomes a dendriform algebra homomorphism.

Therefore, Corollary 113 (and the $\mathcal{A}_{\text {st }} \cong$ QSym $/ \mathcal{K}_{\text {st }}$ isomorphism from Proposition 101) yields the following:

Corollary 116. If a descent statistic st is LR-shuffle-compatible, then its shuffle algebra $\mathcal{A}_{\text {st }}$ canonically becomes a dendriform algebra.

We furthermore have the following analogue of Theorem 95, which easily follows from Theorem 111 and Theorem 112:

Theorem 117. Let st be a descent statistic.

(a) The descent statistic st is left-shuffle-compatible and right-shuffle-compatible if and only if there exist a dendriform algebra $A$ with basis $\left(u_{\alpha}\right)$ (indexed by st-equivalence classes $\alpha$ of compositions) and a dendriform algebra homomorphism $\phi_{\mathrm{st}}:$ QSym $\rightarrow$ $A$ with the property that whenever $\alpha$ is an st-equivalence class of compositions, we have

$$
\phi_{\text {st }}\left(F_{L}\right)=u_{\alpha} \quad \text { for each } L \in \alpha \text {. }
$$

(b) In this case, the $\mathbb{Q}$-linear map

$$
\mathcal{A}_{\mathrm{st}} \rightarrow A, \quad[\pi]_{\mathrm{st}} \mapsto u_{\alpha},
$$

where $\alpha$ is the st-equivalence class of the composition Comp $\pi$, is an isomorphism of dendriform algebras $\mathcal{A}_{\text {st }} \rightarrow A$.

Question 118. Can the $\mathbb{Q}$-algebra Pow $\mathcal{N}$ from Definition 31 be endowed with two binary operations $\prec$ and $\succeq$ that make it into a dendriform algebra? Can we then find an analogue of Proposition 36 along the following lines?

Let $(P, \gamma),(Q, \delta)$ and $(P \sqcup Q, \varepsilon)$ be as in Proposition 36. Assume that each of the posets $P$ and $Q$ has a (global) minimum element; denote these elements by $\min P$ and $\min Q$, respectively. Let $P \prec Q$ be the poset obtained by adding the relation $\min P<$ $\min Q$ to $P \sqcup Q$. Let $P \succ Q$ be the poset obtained by adding the relation $\min P>\min Q$ to $P \sqcup Q$. Then, we hope to have

$$
\begin{aligned}
& \Gamma_{\mathcal{Z}}(P, \gamma) \prec \Gamma_{\mathcal{Z}}(Q, \delta)=\Gamma_{\mathcal{Z}}(P \prec Q, \varepsilon) \quad \text { and } \\
& \Gamma_{\mathcal{Z}}(P, \gamma) \succeq \Gamma_{\mathcal{Z}}(Q, \delta)=\Gamma_{\mathcal{Z}}(P \succ Q, \varepsilon),
\end{aligned}
$$

assuming a simple condition on $\min P$ and $\min Q\left(\right.$ say, $\left.\gamma(\min P)<_{\mathbb{Z}} \delta(\min Q)\right)$. 


\section{Acknowledgments}

We thank Yan Zhuang, Ira Gessel and Sara Billey for helpful conversations and corrections. An anonymous referee has also helpfully pointed out mistakes. The SageMath computer algebra system [13] has been used in finding some of the results below.

\section{References}

[1] Marcelo Aguiar, Nantel Bergeron and Kathryn Nyman, The peak algebra and the descent algebras of types B and D, Trans. Amer. Math. Soc. 356 (2004), pp. 27812824, https://doi.org/10.1090/S0002-9947-04-03541-X.

[2] Sara Billey, Mark Haiman, Schubert polynomials for the classical groups, J. Amer. Math. Soc. 8 (1995), pp. 443-482.

[3] Kurusch Ebrahimi-Fard, Dominique Manchon, Frédéric Patras, New identities in dendriform algebras, Journal of Algebra 320 (2008), pp. 708-727.

[4] Ira M. Gessel, Yan Zhuang, Shuffle-compatible permutation statistics, Advances in Mathematics, Volume 332, 9 July 2018, pp. 85-141. Also available at arXiv:1706.00750v3.

[5] Curtis Greene, Posets of shuffles, Journal of Combinatorial Theory, Series A, Volume 47, Issue 2, March 1988, pp. 191-206.

[6] Darij Grinberg, Dual immaculate creation operators and a dendriform algebra structure on the quasisymmetric functions, version 6, arXiv:1410.0079v6. (Version 5 has been published in: Canad. J. Math. 69 (2017), 21-53.)

[7] Darij Grinberg, Shuffle-compatible permutation statistics II: the exterior peak set [detailed version], detailed version of the present paper. Also available as an ancillary file at arXiv:1806.04114v3.

[8] Darij Grinberg, Victor Reiner, Hopf algebras in Combinatorics, version of 11 May 2018, arXiv:1409.8356v5. See also http://www.cip.ifi.lmu.de/ grinberg/ algebra/HopfComb-sols.pdf for a version that gets updated.

[9] Samuel K. Hsiao, T. Kyle Petersen, Colored Posets and Colored Quasisymmetric Functions, Ann. Comb. 14 (2010), pp. 251-289, https://doi.org/10.1007/ s00026-010-0059-0. See arXiv:math/0610984v1 for a preprint.

[10] Ezgi Kantarcı Oğuz, A Counter Example to the Shuffle Compatiblity Conjecture, arXiv:1807.01398v1.

[11] T. Kyle Petersen, Enriched P-partitions and peak algebras, Advances in Mathematics 209 (2007), pp. 561-610, https://doi.org/10.1016/j.aim.2006.05.016. See arXiv:math/0508041v1 for a preprint.

[12] T. Kyle Petersen, Descents, Peaks, and P-partitions, thesis at Brandeis University, 2006. http://people.brandeis.edu/ gessel/homepage/students/ petersenthesis.pdf 
[13] The Sage Developers, SageMath, the Sage Mathematics Software System (Version 8.0), 2017.

[14] Richard P. Stanley, Ordered Structures and Partitions, Memoirs of the American Mathematical Society, No. 119, American Mathematical Society, Providence, R.I., 1972. http://www-math.mit.edu/ rstan/pubs/pubfiles/9.pdf

[15] Richard Stanley, Enumerative Combinatorics, volume 1, 2nd edition, Cambridge University Press 2012. A preprint is available at http://math.mit.edu/ rstan/ec/.

[16] John R. Stembridge, Enriched P-partitions, Trans. Amer. Math. Soc. 349 (1997), no. 2, pp. $763-788$. 